\title{
DEVELOPMENT OF THE HYPERSPECTRAL NEAR INFRARED SPECTROSCOPY For Monitoring Tissue OXygen Metabolism
}

By

\author{
Reyhaneh Nosrati \\ Bachelor of Biomedical Engineering \\ Islamic Azad University of Tehran, Iran, 2008
}

\begin{abstract}
A thesis
Presented to Ryerson University

In partial fulfillment of the requirements for the degree of

Master of Science

In the Program of

Biomedical Physics
\end{abstract}

Toronto, Ontario, Canada, 2015

CReyhaneh Nosrati 2015 
I hereby declare that I am the sole author of this thesis. This is a true copy of the thesis, including any required final revisions, as accepted by my examiners.

I authorize Ryerson University to lend this thesis to other institutions or individuals for the purpose of scholarly research.

I further authorize Ryerson University to reproduce this thesis by photocopying or by other means, in total or in part, at the request of other institutions or individuals for the purpose of scholarly research.

I understand that this thesis may be made electronically available to the public.

\section{Reyhaneh Nosrati}




\section{Development of the Hyperspectral Near Infrared Spectroscopy \\ for Monitoring Tissue Oxygen Metabolism \\ Master of Science 2015 \\ Reyhaneh Nosrati \\ Biomedical Physics \\ Ryerson University}

\section{Abstract}

Near Infrared Spectroscopy (NIRS) employs optical window of 650-1100 nm to measure the concentrations of different chromophores such as, oxygenated hemoglobin ( $\mathrm{HbO} 2)$, deoxygenated hemoglobin $(\mathrm{Hb})$ and water. In addition, changes in the redox cytochrome $\mathrm{c}$ oxidase (Cyt-ox) can be measured using hyperspectral NIRS. Cyt-ox has a relatively low concentration compared to $\mathrm{HbO}_{2}$ and $\mathrm{HHb}$ therefore its measurements is very challenging.

The main objective of this thesis was to identify the best signal-processing algorithm for deconvolution of the Cyt-ox changes.

In this thesis two different studies have been done; in the first study, functional broadband NIRS during simulated driving (on 16 adult subjects) has been recorded and the best data processing algorithm for deconvolution of the chromophores was identified.

In the second series of experiments broadband NIRS data during cardiac arrest and resuscitation was recorded on 19 pigs. A novel signal-processing algorithm was introduced to deconvolve Cyt-ox.

The Introduction section covers relevant aspects of the theory and mathematical concepts. The Journal Manuscript part contains the two projects mentioned above. The last part is allocated for the overall conclusion of the entire thesis. 


\section{Acknowledgements}

I would like to express my sincere gratitude to my supervisor Dr. Vladislav Toronov for the continuous support of my MSc study and related research, for his motivation, and immense knowledge. His guidance helped me in all the time of research and writing of this thesis. I could not have imagined having a better advisor and mentor for my MSc studies.

Besides my advisor, I would like to thank the rest of my thesis committee: Dr. Raffi Karshafian and Dr. Yuan Xu for their insightful comments and encouragement, but also for the hard question which incented me to widen my research from various perspectives.

My sincere thanks also goes to Prof. Paul Dorian and his team who provided me an opportunity to join their team at St. Michael's Hospital, and who gave access to their high end laboratory and research facilities. Without they precious support it would not be possible to conduct this research.

Finally my warmest thanks must be to my family. Thanks to my husband for providing me with unfailing support and continuous encouragement. This accomplishment would not have been possible without him.

This research has been supported by the Ryerson University Faculty of Engineering, Architecture and Science Dean's Research Fund. 


\section{Dedication}

To my wonderful spouse, Mahdi who supported and inspired me. 


\section{Table of content:}

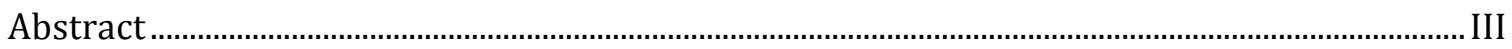

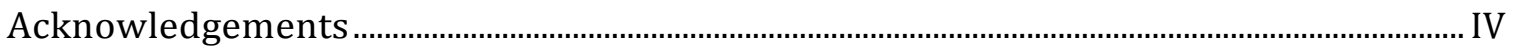

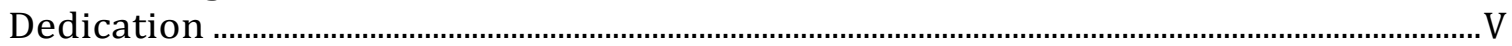

Table of content:

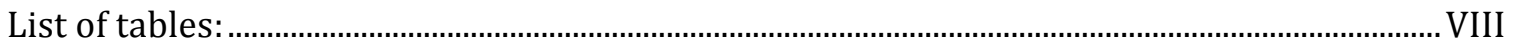

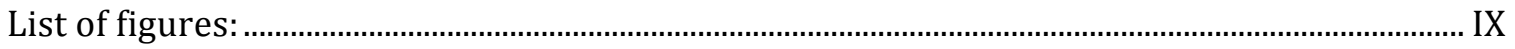

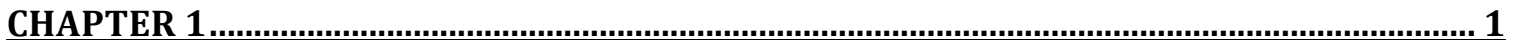

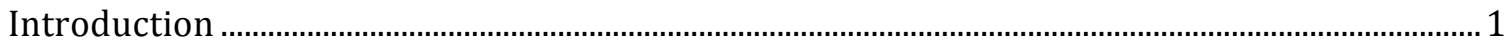

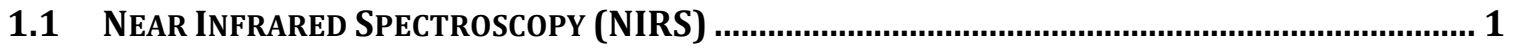

1.1.1 MULTISPECTRAL AND HYPERSPECTRAL TISSUE SPECTROSCOPIC TECHNIQUES................................ 2

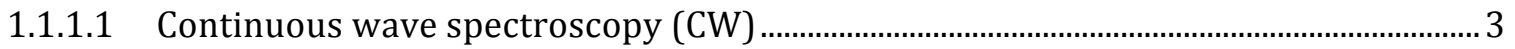

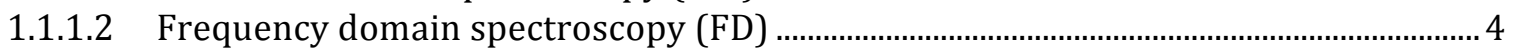

1.1.1.3 Time resolved spectroscopy (TR) …….......................................................................... 4

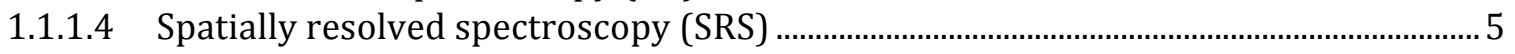

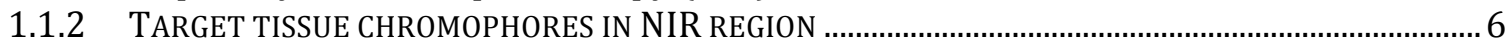

1.1.2.1 Oxygenated and deoxygenated hemoglobin ...................................................................... 6

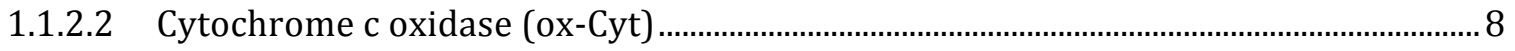

1.1.2.2.1 Aerobic cellular respiration and energy synthesis.......................................................... 8

1.1.3 ABSORPTION MODELING USING MODIFIED BEER-LAMBERT LAW (MBLL) ................................... 11

1.1.4 ABSORPTION MODELING USING DIFFUSION APPROXIMATION.................................................... 14

1.1.4.1 Diffusion equation for a semi-infinite medium; Extrapolated boundary and image

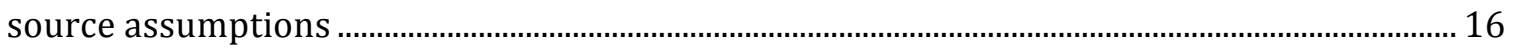

1.1.4.2 Analytical solution to the diffusion equation................................................................ 17

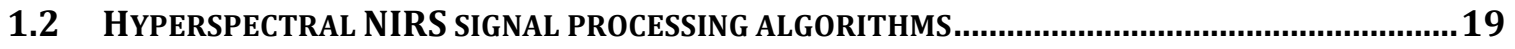

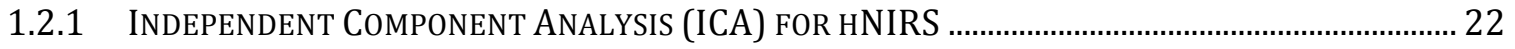

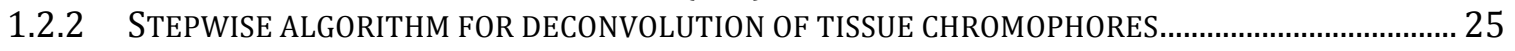

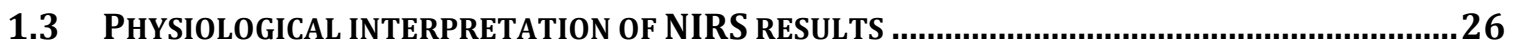

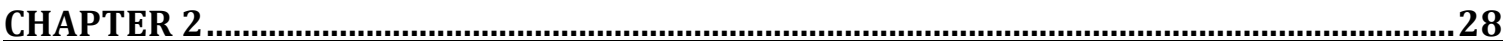

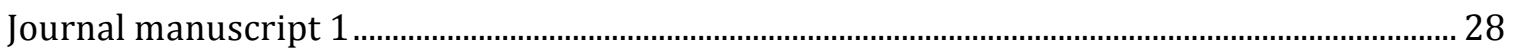

\section{EVENT-RELATED CHANGES OF THE PREFRONTAL CORTEX ACTIVITY DURING

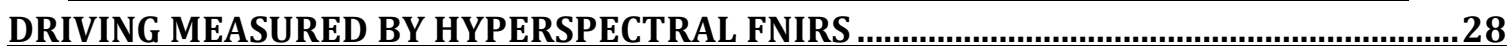

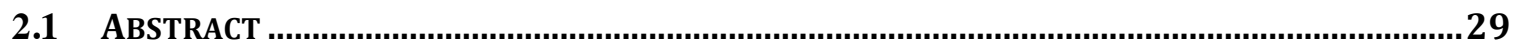

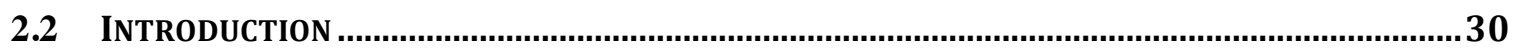

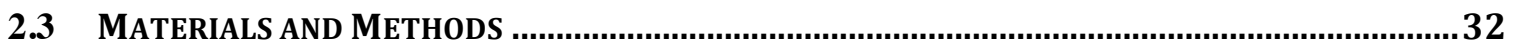

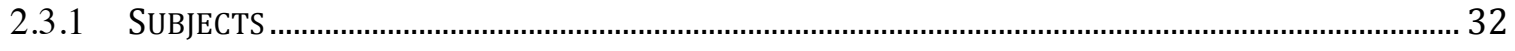

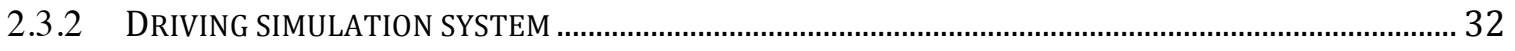

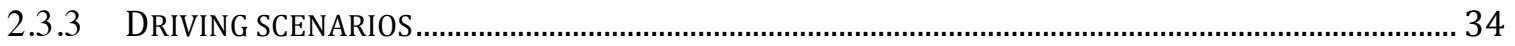

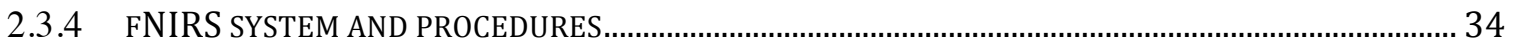

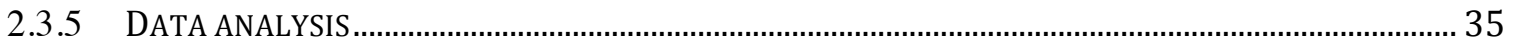

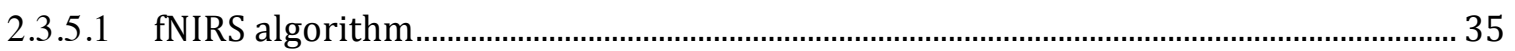

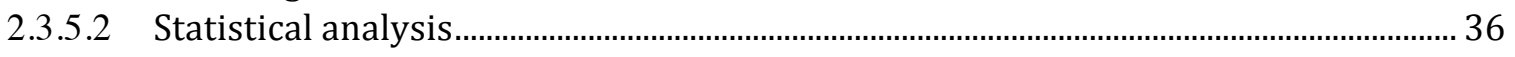

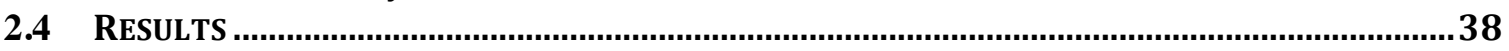


2.4.1 “RIGHT TURN" VS. “RIGHT TURN + DISTRACTION” ................................................................. 38

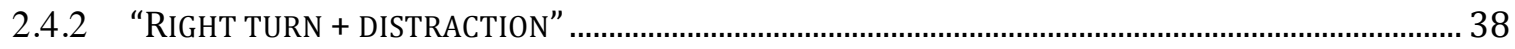

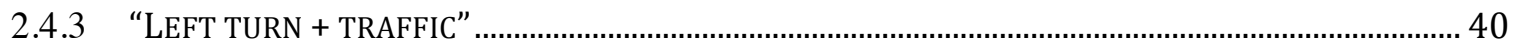

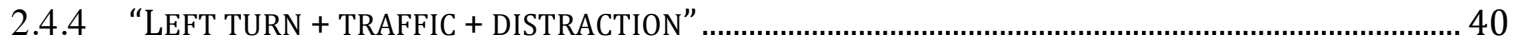

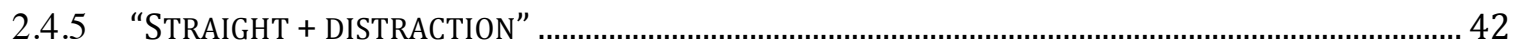

2.4.6 COMPARING DIFFERENT DISTRACTED CONDITIONS ......................................................................... 42

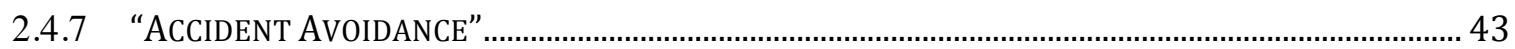

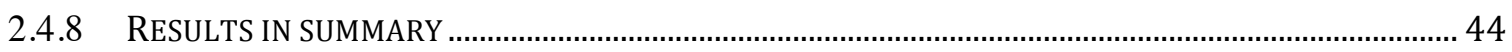

2.5 DISCUSSION

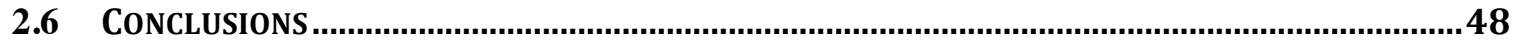

CHAPTER 3

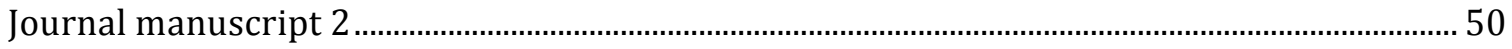

\section{SIMULTANEOUS MEASUREMENT OF CEREBRAL AND MUSCLE TISSUE PARAMETERS DURING CARDIAC ARREST AND CARDIOPULMONARY RESUSCITATION 50}

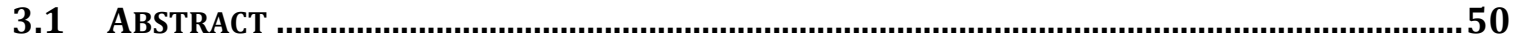

3.2 INTRODUCTION

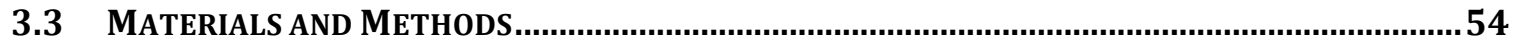

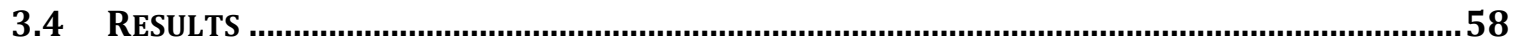

3.4.1 COMPARISON BETWEEN INVASIVE VS. SEMI-INVASIVE CEREBRAL NIRS …..................................... 58

3.4.2 BRAIN AND MUSCLE OXYGEN DELIVERY AND METABOLISM DURING VF AND CPR.......................... 60

3.4.3 BRAIN OXYGENATION AND METABOLISM IN SURVIVAL VS. NON-SURVIVAL ANIMALS ...................... 65

3.4.4 THE EFFECT OF RAPID PACING ON CARDIAC OUTPUT, CEREBRAL OXYGENATION AND METABOLISM 69

3.4.5 THE EFFECT OF $\mathrm{FIO}_{2}$ ON CARDIAC OUTPUT, CEREBRAL OXYGENATION AND METABOLISM............. 71

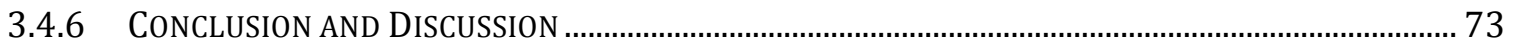

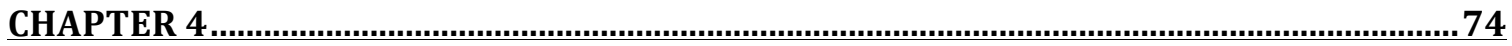

DISCUSSION, CONCLUSION AND FUTURE WORK …......................................................... 74

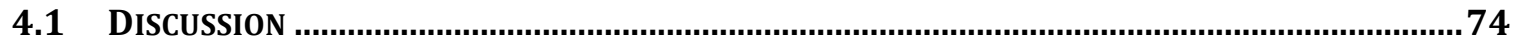

4.2 CONCLUSION

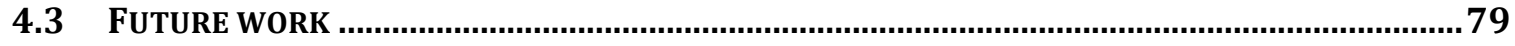

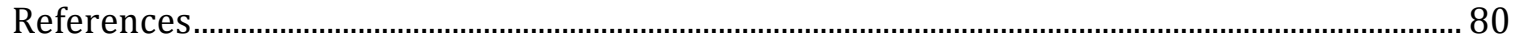




\section{List of tables:}

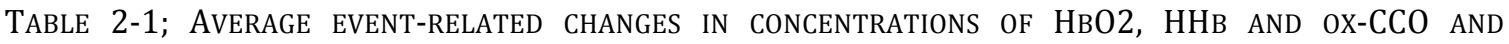
CALCULATED P-VALUE FROM PAIRED-SAMPLE T-TESTS (PERFORMED BETWEEN THE INITIAL MOMENT OF EACH EVENT AND THE MAXIMUM INDUCED CHANGES); ARROWS SHOW THE DIRECTION OF THE AVERAGE CHANGES THAT WERE STATISTICALLY SIGNIFICANT AND THE CHANGES THAT WERE NOT STATISTICALLY SIGNIFICANT ARE SHOWN BY $\approx$.

TABLE 2-2; AVERAGE CONCENTRATION CHANGES AND ONE-WAY ANOVA RESULTS OF ALL DISTRACTED CONDITIONS VS. AVERAGE CHANGES OF ALL NON-DISTRACTED CONDITIONS; POSITIVE MEANS REFLECT AN OVERALL INCREASE AND NEGATIVE MEANS REPRESENT AN OVERALl DECREASE. THE MEAN CHANGES IN RIGHT PFC ARE ALSO SHOWN IN THE BAR GRAPH. ( ${ }^{*}$ IN THE BAR GRAPH OX-CCO CHANGES HAS BEEN MULTIPLIED BY A FACTOR OF 107).

TABlE 2-3; CHANGES FROM THE BASELINE TO THE END OF DROP/RISE AFTER VF INDUCTION ON NIRS MEASURED SIGNALS AND MEAN AORTIC BLOOD PRESSURE. 58

TABLE 2-4; THE IMPACT OF CPR ON DIFFERENT CEREBRAL PARAMETERS MEASURED BY NIRS THROUGH THE SKULL (SEMI-INVASIVELY) AND DURA (INVASIVELY) AND MEAN AORTIC PRESSURE (CHANGES ARE CALCULATED AS THE DIFFERENCE RIGHT BEFORE AND MAXIMUM CHANGES DURING CPR)

TABLE 2-5; THE IMPACT OF VF ON DIFFERENT TISSUE PARAMETERS MEASURED BY NIRS AND HEMODYNAMICS (CHANGES ARE CALCULATED AS THE CHANGES 120 S AFTER INDUCTION OF THE VF MINUS BASELINE VALUES); P-VALUES ARE CALCULATED BETWEEN MUSCLE AND BRAIN (TWO SAMPLE T-TEST, TWO-TAILED, 8 DEGREE OF FREEDOM)

TABLE 2-6; THE IMPACT OF CPR ON DIFFERENT TISSUE PARAMETERS MEASURED BY NIRS AND HEMODYNAMICS (CHANGES ARE CALCULATED AS THE MAXIMUM CHANGES DURING CPR MINUS SIGNAL AMPLITUDE BEFORE CPR); P-VALUES ARE CALCUlated BETWEen MUSCLE AND BRAIN (TWO SAMPLE T-TEST, TWO-TAILED, 8 DEGREE OF FREEDOM)

TABLE 2-7; THE IMPACT OF DIFFERENT PACING RATE ON CEREBRAL HEMODYNAMICS AND METABOLISM; $\Delta \mathrm{P} / \Delta \mathrm{F} / \Delta \mathrm{C}$ REPRESENT THE MAXIMUM DROP IN BLOOD PRESSURE/FLOW OR CONCENTRATION FROM THE BASELINE VALUE DUE TO CHANGING PACING RATE AND $\triangle$ T REPRESENTS THE RECOVERY TIME FROM THE ONSET OF EACH PACING RATE TO THE INITIAL VALUE.

TABLE 2-8; THE EFFECT OF FIO 2 ALTERNATION ON HEMODYNAMICS AND CEREBRAL OXYGENATION AND METABOLISM. THE CHANGES ARE CALCULATED AS THE DIFFERENCE BETWEEN THE FIRST AND LAST MOMENT OF INHALED $\mathrm{O}_{2}$ LEVEL. 


\section{List of figures:}

FIGURE 1-1, SRS SYSTEM SCHEMATIC PRINCIPAL AND A SAMPLE SRS PROFILE MEASURED WITH A CONTACT PROBE [12]

FIGURE 1-2; THE SPECIFIC EXTINCTION COEFFICIENTS OF HBO2, HB AND OXIDIZED MINUS REDUCED CYTOCHROME OXIDASE;

FIGURE 1-3; ELECTRON TRANSPORT CHAIN MECHANISM WITHIN INNER MEMBRANE OF MITOCHONDRIA .....................10

FIgURE 1-4, SCHEMATIC OF LIGHT ABSORPTION IN A NON-SCATTERING MEDIUM THAT CAN BE DESCRIBED BY THE BEER-LAMBERT LAW

FIGURE1-5; SCHEMATICS OF THE EXTRAPOLATED BOUNDARY AT - $Z_{\mathrm{B}}$ AND IMAGINARY SOURCE AT Z $\mathrm{Z}_{0}$ CONDITIONS IN A HIGHLY SCATTERING MEDIUM TO MEET THE DIFFUSION APPROXIMATION REQUIREMENTS..................................17

FIGURE 1-6; HYPERSPECTRAL FUNCTIONAL NIRS DATA PROCESSING ALGORITHM .....................................................21

FIGURE 1-7; HYPERSPECTRAL NON-FUNCTIONAL NIRS DATA PROCESSING ALGORITHMS...............................................22

FIGURE 1-8, EXTINCTION SPECTRA OF MAIN CHROMOPHOES IN NIR REGION OF THE EM SPECTRUM ...........................26

FIGURE 2-1-A) DRIVING SIMULATOR AND FNIRS MEASUREMENT APPARATUS; B) EVENT-RELATED DRIVING SCENARIO (SECOND DRIVING SCENARIO INCLUDING DISTRACTION) ….................................................................................33

FIGURE 2-2; INDIVIDUAL EVENT-RELATED CHANGES DURING A) RIGHT TURN B) DISTRACTED RIGHT TURN.................39

FIGURE 2-3; AVERAGE TRACES REPRESENTING CONCENTRATION CHANGES DURING A) RIGHT TURN; B) DISTRACTED RIGHT TURN.

FIGURE 2-4; INDIVIDUAL EVENT RELATED CONCENTRATION CHANGES DURING A) LEFT TURN IN TRAFFIC; B) DISTRACTED LEFT TURN IN TRAFFIC

FiguRE 2-5; AVERAGE TRACES OF CONCENTRATION CHANGES DURING A) LEFT TURN IN TRAFFIC; B) DISTRACTED TURN IN TRAFFIC .

FIGURE 2-6; A) INDIVIDUAL AND B) AVERAGE CHANGES DURING DISTRACTED STRAIGHT DRIVING ...............................42

FIGURE 2-7; COMPARING THE EFFECT OF DISTRACTION DURING DIFFERENT DRIVING TASKS IN A) RIGHT PFC AND B) LEFT PFC OF A RANDOM SUBJECT C) INDUCED CHANGES DURING DIFFERENT NON-DISTRACTED DRIVING TASKS ON THE SAME SUBJECT .

FigURE 2-8; AVERAGE INDUCED CHANGES DURING NON-DISTRACTED CONDITIONS VERSUS DISTRACTED CONDITIONS; MEAN CHANGES HAVE BEEN CALCULATED WITH ANOVA BETWEEN ALL DISTRACTED CONDITIONS (INCLUDING LEFT TURN + DISTRACTION, STRAIGHT + DISTRACTION AND RIGHT TURN + DISTRACTION) AND NONDISTRACTED CONDITIONS (INCLUDING LEFT TURN, STRAIGHT AND RIGHT TURN); * OX-CCO VALUES HAVE BEEN MULTIPLIED BY 10 ....

FIGURE 3-1; [HBO2], [HНB], [CYT-OX] AND TSO2 CHANGES MEASURED BY NIRS BOTH INVASIVELY (DURA) AND SEMI-INVASIVELY (SKULL) AND MEAN AORTIC BLOOD PRESSURE DURING CARDIAC ARREST AND RESUSCITATION

.60

Figure 3-2; Cerebral AND MUSClE TISSUE heMOdYNAMiCS, [HHB], [HBO2], $\Delta$ [CYT-OX] AND TSO2 CHANGES DURING VENTRICULAR FIBRILLATION AND RESUSCITATION PROCESS INCLUDING CPR, EPINEPHRINE (EPI) AND DEFIBRILLATION (DIF); THE SHOWN DATA ARE FROM A RANDOM NON-SURVIVAL ANIMAL (JULY 24 2014) .....64

FIGURE 3-3; COMPARING A SURVIVAL CASE VS. A NON-SURVIVAL CASE; HEMODYNAMICS AND NIRS MEASURED CEREBRAL [HHB], $\left[\mathrm{HBO}_{2}\right], \Delta[\mathrm{CYT}-\mathrm{OX}]$ AND $\mathrm{TSO}_{2}$ CHANGES DURING VENTRICULAR FIBRILLATION AND RESUSCITATION.

FIGURE 3-4; COMPARING A SURVIVAL (PLOTS ON LEFT COLUMN) CASE VS. A NON-SURVIVAL (PLOTS ON THE RIGHT COLUMN) CASE; HEMODYNAMICS AND NIRS MEASURED CEREBRAL [HHB], [HBO $\mathrm{HB}_{2}, \Delta$ [CYT-OX] AND TSO 2 CHANGES DURING VENTRICULAR FIBRILLATION AND RESUSCITATION.

FIGURE 3-5; THE EFFECT OF DIFFERENT PACING RATES ON HEMODYNAMICS AND IIRS MEASURED CEREBRAL OXYGENATION AND METABOLISM LEVEL ……................................................................................................ 71

FIGURE 3-6; THE EFFECT OF FIO2 ALTERNATION (30\% AND 14\% 02 RESPECTIVELY) ON HEMODYNAMICS AND NIRS MEASURED CEREBRAL OXYGENATION AND METABOLISM LEVEL. 


\section{CHAPTER 1}

\section{Introduction}

\subsection{Near Infrared Spectroscopy (NIRS)}

NIRS is generally probing the concentration of different chromophores in biological tissue using near infrared region of the electromagnetic spectrum. NIRS can detect minor changes induced by activation or any other stimulation in the brain, muscle or other tissues. The NIR light is emitted directly on the tissue surface by a source optode and a detector optode placed at a distance from the source recieves the backscattered light. Based on the known extinction coefficient of different chromophores at different wavelengths and the amount of absorption of each wavelength, concentration of target substances is determined.

It was first reported in 1977 by FF Jobsis [1] that in the optical window of the near infrared, tissue oxygen saturation and metabolism could be measured noninvasively. They showed that NIR light could diffuse through the intact scalp and skull of an adult human to monitor oxygenated and deoxygenated hemoglobin concentrations in the brain [2].

M Ferrari [3] for the first time measured cerebral oxygenation using NIRS in 1985.

In 1988 M Cope and D Delpy [4] proposed modified Beer-Lambert law (MBLL) to quantify absorption changes in the cerebral tissue by adding a factor called "differential path length factor" (DPF) to correct for the increased travelling distance 
of the light in a highly scattering medium such as tissue, to the original BeerLambert law.

Randall Barbour [5], Briton Chance [6], Arno Villringer [7], Enrico Gratton, and others further developed NIRS techniques.

Near infrared spectroscopy (NIRS) employs the near-infrared region of the electromagnetic spectrum $(650-1100 \mathrm{~nm})$ for real-time measurement of living tissue hemodynamics and metabolism.

NIRS is a non-invasive and inexpensive imaging method for quantification of different tissue chromophores based on the regional absorption of the NIR light within biological tissue. NIRS takes advantage of the optical window in which biological tissues are mostly transparent to this wavelength range, except for a few chromophores. Water as the main component of the tissues has minor absorption within NIR range hence the NIR light is mainly absorbed by certain chromophores that represent hemodynamic responses and metabolism level of the tissue such as oxygenated hemoglobin, deoxygenated hemoglobin $\left(\mathrm{HbO}_{2}\right.$ and $\mathrm{HHb}$ respectively) and cytochrome c oxidase (cyt-ox).

\subsubsection{Multispectral and hyperspectral tissue spectroscopic techniques}

There are multispectral and hyperspectral tissue spectroscopic techniques. The multispectral systems include typically use two wavelengths of NIR light, such as $690 \mathrm{~nm}$ and $830 \mathrm{~nm}$. One wavelength is more sensitive to oxyhemoglobin while another is more sensitive to deoxyhemoglobin. Rare systems use upt to five different NIR wavelengths. The typical detectors in multispectral NIRS devices are either photomultiplier tubes or 
avalanch photodiodes. The hyperspectral techniques employs a quasicontinuum spectrum measured by a spectrometer.

\subsubsection{Continuous wave spectroscopy $(\mathrm{CW})$}

$\mathrm{CW}$ approach employs a NIR source with a constant frequency and amplitude. Changes in light intensity after travelling through tissue reflects the concentration of different substances that can be quantified based on the known extinction coefficients using MBL law or diffusion approximation theory. CW systems could be multi-wavelength (using two to four discrete wavelengths) or hyperspectral or broadband (using spectral band between $650-1100 \mathrm{~nm}$ ). Multispectral NIRS systems use a minimum of two wavelengths and are capable of monitoring $\mathrm{HbO} 2$ and $\mathrm{HHb}$ changes. Broadband or hyperspectral systems provide a much better spectral resolution compared to multi-wavelength systems. The CW method basically calculates relative concentration changes not absolute concentrations. Multispectral $\mathrm{CW}$ technique does not provide information about photon path length within tissue and absolute concentrations are proportional to an unknown path-length. In many studies that employ MBLL for multispectral CW NIRS absolute quantification of hemoglobin concentrations is performed using estimations of photon path length calculated with Monte Carlo simulation or average values calculated by other NIRs techniques such as frequency domain. Hyperspectral NIRS (hNIRS) can measure both absolute values [PMCID: PMC3493236] of and relative changes in the chromophore concentrations. It is the goal of this thesis to show the advantages of CW hNIRS to noninvasively measure changes in physiological parameters. 


\subsubsection{Frequency domain spectroscopy (FD)}

In frequency domain (FD) systems employ a laser diode, LED or modulated white light sources. The detectors are photomultiplier tubes (PMTs). The FD system is capable of measurement of absolute values of $\mathrm{HbO}_{2}$ and $\mathrm{HHb}$. The source and PMTs are coupled to fiber optics. Changes in the back-scattered signal's amplitude and phase provide information on changes in $\mathrm{HbO}_{2}$ and $\mathrm{HHb}$. The main advantage of FD systems is the measurement of the absolute values of absorption $\left(\mu_{\mathrm{a}}\right)$ and reduced scattering $\left(\mu_{s}^{\prime}\right)$ coefficients that quantify the absolute concentrations of $\mathrm{HbO}_{2}$ and $\mathrm{HHb}$. Because for FD technique modulated laser sources and sophisticated phasic measurements are required, they are more technically complex and expensive than CW systems.

\subsubsection{Time resolved spectroscopy (TR)}

In the TR technique a very short pulse (in order of pico/femtosecond) of NIR light is injected and after detection of the backscattered photon the photon path length is calculated using the flight time and speed of light in biological tissue. The TR method relies on the ability of resolving changes in light intensity with extremely high temporal resolution that require short pulse laser source (picosecond to femtosecond) and highspeed detector that makes it the most expensive and complex spectroscopic technique. Hemodynamic changes are determined using the attenuation, decay, and time profile of the detected pulse. 


\subsubsection{Spatially resolved spectroscopy (SRS)}

Spatially resolved spectroscopy (SRS) employs continuous wave NIR light source (usually several laser sources with different wavelengths) and has the advantage of being a low cost system. The SRS system performs multiple measurements at different sourcedetector distances and does not require information about photon path length. The main advantage of SRS is the real-time calculation of the tissue oxygenation index (TOI), which is the ratio of oxygenated to the total hemoglobin. Slope of light attenuation/reflectance versus detector distance from the incident light that is proportional to the penetration depth and number of interactions, is calculated to obtain sufficient information to separate the light absorption and scattering properties and eventually TOI is determined based on the diffusion approximation theory [11]. This technique is mostly used for cerebral tissue oxymetry providing TOI information.
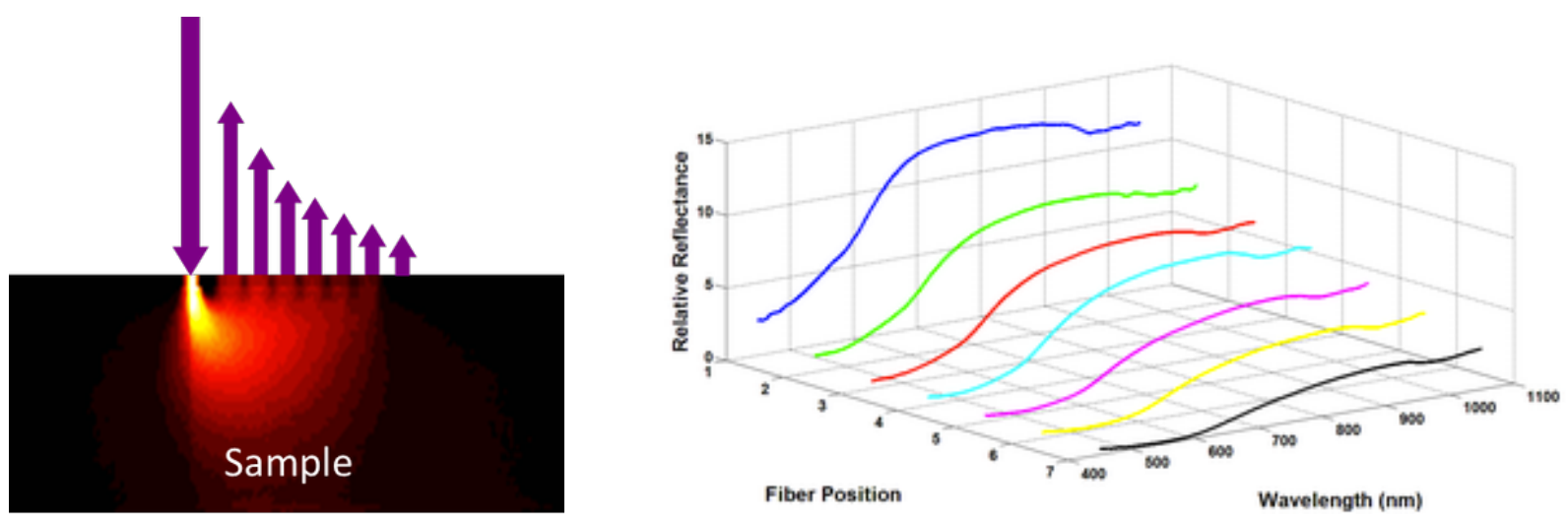

Figure 1-1, SRS system schematic principal and a sample SRS profile measured with a contact probe [12] 


\subsubsection{Target tissue chromophores in NIR region}

The main NIR absorber chromophores that reflect the level of tissue oxygen delivery and oxygen metabolism are oxyhemoglobin $\left(\mathrm{HbO}_{2}\right)$, deoxyhemoglobin $(\mathrm{HHb})$ and redox state of cytochrome oxidase (ox-cyt). Fig. 2 shows the specific extinction coefficients of main NIR absorber chromophores.

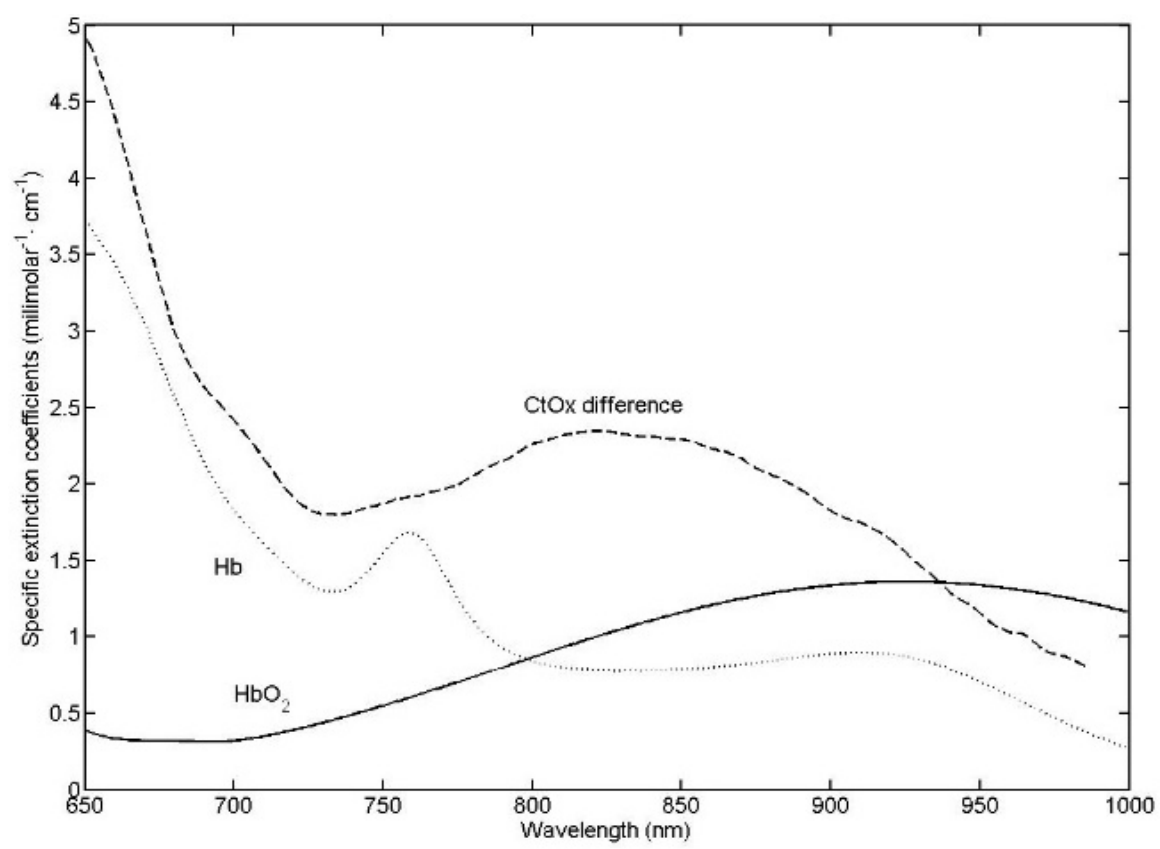

Figure 1-2; The specific extinction coefficients of $\mathrm{HbO2}, \mathrm{Hb}$ and oxidized minus reduced cytochrome oxidase;

\subsubsection{Oxygenated and deoxygenated hemoglobin}

Hemoglobin $(\mathrm{Hb})$ is a metal-based protein pigment in red blood cells. The main role of hemoglobin is oxygen transport. Each hemoglobin molecule contains four heme $(\mathrm{Fe})$ centers that each can bind to one $\mathrm{O}_{2}$ molecule. Hemoglobin molecule has a very high oxygen-binding capacity $(1.34 \mathrm{~mL} / \mathrm{gr})$ which makes the total blood oxygen capacity seventy-fold compared to dissolved oxygen in the blood without hemoglobin [13]. The 
mammalian hemoglobin molecule can carry up to four oxygen molecules [14]. Oxygen molecules bind to the $\mathrm{Fe}$ components of the $\mathrm{Hb}$ in the pulmonary capillaries adjacent to the alveoli of the lungs and then travel through the blood stream to reach peripheral tissues to be utilized as the terminal electron acceptor in the aerobic ATP synthesis process by which $95 \%$ of the required energy for the cells is provided.

Hemoglobin exists in two forms of tense and relaxed, determining the level of $\mathrm{Hb}$ oxygen affinity. Higher oxygen affinity in tense form results in lower oxygen unloading in the cells and vise versa; the main factors that dramatically reduce oxygen affinity hence increase $\mathrm{O}_{2}$ unloading for use in peripheral tissues are low $\mathrm{pH}$, high $\mathrm{CO}_{2}$ and high 2,3 BPG [15]. Carbon dioxide level changes the intracellular $\mathrm{pH}$ through formation of bicarbonate ion. 2,3 BPG is formed during anaerobic glycolysis in red blood cells that do not have mitochondria; concentration of 2-3 BPG increases in RBC when $\mathrm{O}_{2}$ concentration decreases in the RBCs so called hypoxemia condition. [16].

As shown in Fig.1-2 the extinction spectra of oxygenated hemoglobin $\left(\mathrm{HbO}_{2}\right)$ and deoxygenated hemoglobin $(\mathrm{Hb} / \mathrm{HHb})$ are different. $\mathrm{HbO}_{2}$ has an absorption peak around $940 \mathrm{~nm}$ and $\mathrm{HHb}$ 's absorption peak is around $650 \mathrm{~nm}$. Isobestic point is the wavelength at which $\mathrm{HbO}_{2}$ and $\mathrm{HHb}$ have equal molar extinction coefficients that is around $800 \mathrm{~nm}$ in biological tissue. Isobestic point is utilized to calculate total tissue hemoglobin concentration. In order to be sensitive to $\mathrm{HbO}_{2}$ and $\mathrm{HHb}$ concentrations individually, multi-wavelength NIRS systems employ two laser sources with wavelengths between 700 and $850 \mathrm{~nm}$ (usually 700 and $850 \mathrm{~nm}$ ) at which $\mathrm{HbO}_{2}$ and $\mathrm{HHb}$ extinction spectra are maximally separated and $\mathrm{H}_{2} \mathrm{O}$ extinction spectrum has minimal overlap with them [17]. In addition to measure total $\mathrm{Hb}$ concentration changes directly, the isobestic wavelength 
is also employed in multi-wavelength systems. In hyperspectral NIRS around 1000 wavelengths are utilized (between 650 and $1100 \mathrm{~nm}$ with spectral resolution of $0.5 \mathrm{~nm}$ ) to deconvolute low concentration chromophores such as [ox-Cyt] more effectively.

\subsubsection{Cytochrome c oxidase (ox-Cyt)}

One of the tissue chromophores that reflects intracellular oxygen metabolism is the redox state of the cytochrome $\mathrm{c}$ oxidase ( $\Delta[\mathrm{ox}-\mathrm{Cyt}])$; it has a strong and broad absorption peak around $800 \mathrm{~nm}$. The concentration of ox-Cyt is less than $10 \%$ of that of hemoglobin and [16]. Monitoring $\Delta[\mathrm{ox}-\mathrm{Cyt}]$ is very challenging compared to that of hemoglobin due to its low concentration and it has been reported by Matcher and colleagues [17] that calculating $\Delta[\mathrm{ox}$-Cyt] using a multispectral system with a limited number of wavelengths can give spurious measurements and therefore it is recommended to use hyperspectral NIRS system.

Cyt-ox is responsible for 95\% of the cell oxygen metabolism and energy (ATP) synthesis; in the following section different stages by which ATP molecules are synthesized will be explained and the significant role of the Cyt-ox will be discussed.

\subsection{Aerobic cellular respiration and energy synthesis}

Adenosine triphosphate (ATP) is the molecular unit of intracellular energy packages. ATPs have the main role in energy requiring metabolic reactions by which they are consumed. ATP molecules are generated from ADP through an energy consuming process. ATPs are produced through three different mechanisms.

The first process occurring in the cytoplasm is anaerobic glycolysis by which each glucose molecule is transformed into two pyruvate, 2 reducing agents (NADH) and two ATP molecules are formed (per glucose) that is about $5 \%$ of the total glucose energy 
potential. Anaerobic glycolysis is the dominant energy production process during hypoxia and works during short, intense exercise, providing energy for a short period (10 seconds to 2 minutes). ATP production by this approach is about 100 times faster than the other two mechanisms.

The second mechanism is called citric acid cycle or Krebs cycle occurring in the matrix of the mitochondria. In this process the generated pyruvate molecules from glycolysis enter the mitochondrial matrix and undergo complex chemical reactions by which eventually one ATP molecule (per pyruvate molecule) is produced; the most important products of Krebs cycle are reducing agents $\left(8 \mathrm{NADH}\right.$ and $\left.2 \mathrm{FADH}_{2}\right)$ that trigger the third and most important ATP synthesis mechanism.

The third and most important mechanism that generates almost $90 \%$ of the required ATP molecules for the cells is called phosphorylation or electron transport chain (ETC). ETC occurs in the inner membrane of the mitochondria and is composed of a series of compounds that transfer electrons from an electron donor or reducing agent to electron acceptor through redox reactions. Fig. 1-3 shows the ETC and ATP synthesis within mitochondrial membrane. Each reducing agent generated during glycolysis and Krebs cycle donates two electrons to the first component of the chain and these electrons are sequentially transferred between different compartments of the chain including: complex I (NADH dehydrogenase), Ubiquinone, cytochrome b-c $c_{1}$ complex, Cytochrome $\mathrm{c}$ and Cytochrome c oxidase respectively [18]. The terminal electron acceptors are oxygen molecules that receive transferred electrons and transform into water molecules along with hydrogen ions. During this phenomenon electron carrier molecules use the energy from electrons 
to actively pumps protons from the matrix into the intermembrane space, this series of hydrogen pumping steps creates a proton concentration gradient; protons can diffuse back across the membrane through ATP synthase enzyme (channel) located in the inner membrane space. The ATP synthase enzyme used the energy of the protons to bind a phosphate group to the ADP producing ATP. Each NADH yields 3 ATP and FADH2 yields 2 ATP; The ETC greatly increases the yield of ATP from the breakdown of glucose. Overall each ETC generates 32 ATP per glucose molecule breakdown.

Cytochrome c oxidase (CCO) is the final electron acceptor in electron transport chain; it has four metal centers and among those one copper center $\left(\mathrm{Cu}_{\mathrm{A}}\right)$ has a distinct absorption spectrum in NIR range (peak at $820 \mathrm{~nm}$ ). Total concentration of CCO is almost constant during a limited time but it changes between reduced and oxidized states; the spectrum that is used for deconvolution of the cytochrome oxidase changes as shown in Fig. 1-2, is the difference absorption spectrum of the oxidized and reduced enzyme [15].

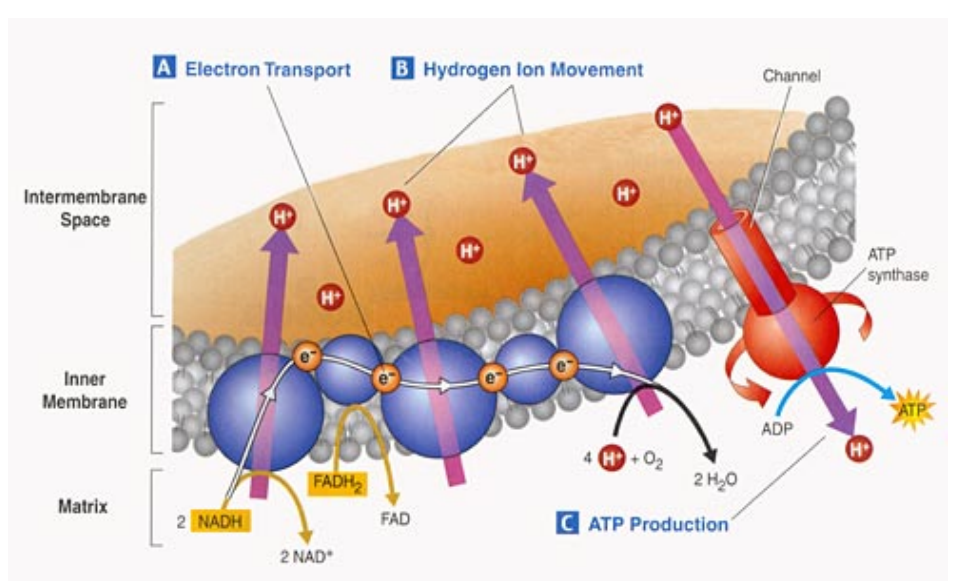

Figure 1-3; Electron transport chain mechanism within inner membrane of mitochondria 


\subsubsection{Absorption modeling using modified Beer-Lambert law (MBLL)}

The most commonly used model to quantify absorption changes from CW NIRS data is the modified Beer-Lambert law (MBLL) [19].

Fig. 1-4 shows a simple diagram of light travelling in a transparent (non-scattering) medium that can be described by Beer-Lambert Law.

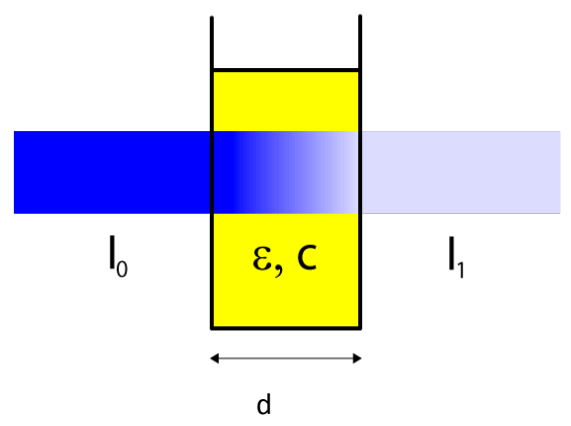

Figure 1-4, Schematic of light absorption in a non-scattering medium that can be described by the Beer-Lambert Law

Absorbance of the light travelling in a absorbing medium is described as following:

$\boldsymbol{A}_{\lambda}=\ln \left(\frac{\boldsymbol{I}_{\mathbf{0}}}{\boldsymbol{I}}\right) \quad[\boldsymbol{E q} .1-1]$

where $A_{\lambda}$ is absorbance of wavelength $\lambda, I_{0}$ is the light intensity transmitted by the medium and I is the light intensity received by the medium

The Beer's law [20] stated that absorbance is directly proportional to the concentration of substances in a medium in which the light is travelling. The general equation for Beer's law is:

$\boldsymbol{A}_{\lambda}=\boldsymbol{\varepsilon}_{\lambda} \times \boldsymbol{c} \times \boldsymbol{d} \quad[\boldsymbol{E q} .1-2]$

where $\boldsymbol{A}_{\lambda}$ is absorbance of wavelength $\lambda, \boldsymbol{\varepsilon}_{\lambda}$ is the molar extinction coefficient of wavelength $\lambda$, $c$ is the concentration and $d$ is the light's travelling distance (thickness of the sample)

Lamberts law [21] stated that absorbance is directly proportional to the distance that light is travelling described by the following equation: 
$A_{\lambda}=\mu_{a(\lambda)} \times d \quad[E q .1-3]$

where $\boldsymbol{A}_{\lambda}$ is the absorbance, $\mu_{a}$ is the absorption coefficient and $d$ is the light pathlength

When Equations 1-1 and 1-2 are combined the Beer-Lambert law is obtained which relates the absorbance of the light to the optical properties and concentration of different substances in a transparent medium.

$A_{\lambda}=\ln \left(\frac{I_{0}}{I}\right)=\varepsilon_{\lambda} \times c \times d \quad[E q .1-4]$ (Beer-Lambert Law)

where $A_{\lambda}$ is absorbance of wavelength $\lambda, I_{0}$ is the light intensity transmitted by the medium, $I$ is the light intensity received by the medium, $\boldsymbol{\varepsilon}_{\lambda}$ is the molar extinction coefficient of $\lambda, c$ is the concentration and $d$ is the light's travelling distance

Beer-Lambert equation works only for a non-scattering medium in which the travelling distance of the light is equal to the straight distance between source and detector however in a highly scattering medium such as biological tissue due to scattering the travelling distance of the light is significantly greater than the distance between source and detector. The Modified Beer-Lambert Law (MBLL), which is the basis of the CW NIRS, introduces a new factor called "differential path length factor (DPF) to correct for the increased path length of the light due to scattering. According to the MBLL light attenuation is affected by both scattering and absorption. MBLL is described by the following equation:

$A_{\lambda}=\ln \left(\frac{I_{0}}{I}\right)=\varepsilon_{\lambda} \times c \times d \times D P F+G \quad[E q .1-5]$ (Modified Beer-Lambert Law) where $A_{\lambda}$ is absorbance of wavelength $\lambda, I_{0}$ is the light intensity transmitted by the medium, I is the light intensity received by the medium, $\boldsymbol{\varepsilon}_{\lambda}$ is the molar extinction coefficient of $\lambda, c$ is the concentration, $d$ is the light's travelling distance, DPF is the differential pathlength factor and $G$ is the scattering loss factor which is generally unknown

In order to apply MBLL model for modeling the absorption, $\mathrm{d}$ or the source-detector distance must be greater than mean free pathlength of the photons within tissue. Scattering loss $(\mathrm{G})$ accounts for the photon loss due to scattering and is not known in CW 
NIRS using MBLL model hence absolute concentrations are not derivable and only changes in concentrations can be quantified.

Changes in concentration $(\Delta[\boldsymbol{c}])$ of different chromophores are calculated with respect to the changes in absorbance at different time point (usually different time points are compared to a unit reference point) as described by the following equation:

$A_{\lambda}-A_{(0) \lambda}=\ln \left(\frac{I_{0}}{I}\right)=\varepsilon_{\lambda} \times \Delta[c] \times d \times D P F \quad[E q .1-6]$

In the multi wavelength CW NIRS approach, according to Eq.1-6, for each employed wavelength at each time point one equation is obtained and eventually for every time point depending on the number of wavelengths two to five equations are obtained.

For example in the simplest case using two wavelengths (one for $\mathrm{HbO}_{2}$ and the other for $\mathrm{HHb}$ ) we have two equations for every time point:

$$
\begin{aligned}
\Delta \boldsymbol{A}_{\lambda \mathbf{1}} & =\left[\varepsilon_{\lambda 1}^{\mathrm{HbO}_{2}} \times \Delta[\boldsymbol{c}]_{\boldsymbol{H b o}_{2}}+\varepsilon_{\lambda 1}^{H H b} \times \Delta[\boldsymbol{c}]_{H H \boldsymbol{b}}\right] \times \boldsymbol{d} \times \boldsymbol{D P F}(\boldsymbol{\lambda}) \\
\Delta \boldsymbol{A}_{\lambda 2} & =\left[\varepsilon_{\lambda 2}^{H b O_{2}} \times \Delta[\boldsymbol{c}]_{\boldsymbol{H b o}_{2}}+\varepsilon_{\lambda 2}^{H H b} \times \Delta[\boldsymbol{c}]_{\boldsymbol{H} \boldsymbol{b}}\right] \times \boldsymbol{d} \times \boldsymbol{D P F}(\boldsymbol{\lambda})
\end{aligned}
$$

As mentioned previously, molar extinction coefficients $\left(\varepsilon_{\lambda}\right)$ are known for different chromophores at different wavelengths and $\Delta A$ is also measured with spectrometer hence for each time point we have two equations and two unknowns that can be calculated by simply using following matrices and solving for $\Delta[c]$ matrix:

$$
\left[\begin{array}{l}
\Delta A_{\lambda 1} \\
\Delta A_{\lambda 2}
\end{array}\right]=\left[\begin{array}{cc}
\varepsilon_{\lambda 1}^{H b O_{2}} L & \varepsilon_{\lambda 1}^{H H b} L \\
\varepsilon_{\lambda 2}^{H b O_{2}} L & \varepsilon_{\lambda 2}^{H H b} L
\end{array}\right] \times\left[\begin{array}{c}
\Delta[C]_{H b O_{2}} \\
\Delta[C]_{H H b}
\end{array}\right] \text { where: } L=d \times D P F(\lambda) \quad[\boldsymbol{E q} . \mathbf{1}-\mathbf{8}]
$$

Solving system of equation using matrices for multi wavelength systems is convenient and does not require time consuming mathematical algorithm however in hyperspectral NIRS signal processing algorithms instead of solving system of equations it is more 
convenient to estimate the concentration changes by multi-linear least-square fitting of the absorbance changes to the molar extinction coefficients:

$$
\varepsilon \cdot d \cdot D P F \stackrel{\text { multi-linear-fit }}{\longrightarrow}-\ln \left[\frac{I_{(t) \lambda}}{I_{(r e f) \lambda}}\right]
$$

The coefficient calculated by the data fitting must be divided by the $d \times D P F$ to find the concentration changes. DPF is a function of the wavelength and can be measured experimentally as described in [22].

The quality of the data fit at every time point could be assessed through comparing measured absorbance with the total calculated absorbance (summation of different chromophores). In this thesis different algorithms have been assessed to improve the quality of the data fitting.

Two assumptions are made to apply MBLL for CW-NIRS: firstly tissue absorption changes homogeneously, and secondly the scattering loss is constant. However in reality absorption often changes non-homogeneously, therefore MBLL underestimates the changes in concentrations and calculated values are influenced by the change in the concentration of other chromophores (cross-talk between chromophores) [23].

\subsubsection{Absorption modeling using diffusion approximation}

Photon transport in biological tissue can be modeled numerically with Monte Carlo simulations or analytically by the radiative transfer equation (RTE). In order to model optical properties of the tissue and eventually calculate concentration changes of different chromophores, we need to solve RTE, solving RTE is very complicated though to reduce the number of variables and find an analytical solution to the RTE, some approximations have been introduced and RTE has been reduced to diffusion approximation. 
RTE is a differential equation describing radiance $L(\vec{r}, \hat{s}, t)$. Basically RTE is derived from the law of conservation of energy. RTE states that a beam of light loses energy through divergence, scattering and absorption (extinction) and gains energy from light sources in the medium and scattering directed towards the beam. Optical properties of the tissue such as refractive index $n$, absorption coefficient $\mu_{a}$, scattering coefficient $\mu_{s}$ and scattering anisotropy $g$ are assumed to be time-independent but they are not assumed spatially homogeneous. Elastic scattering is assumed for all scattering events. RTE equation or so called Boltzmann equation is written as [24]:

$\frac{\partial L(\vec{r}, \hat{s}, t) / c}{\partial t}=-\hat{s} . \nabla L(\vec{r}, \hat{s}, t)-\mu_{t} L(\vec{r}, \hat{s}, t)+\int_{4 \pi} L(\vec{r}, \hat{s}, t) P\left(\hat{s}^{\prime}, \hat{s}\right) d \Omega^{\prime}+S(\vec{r}, \hat{s}, t) \quad[E q .1-9]$ Where:

- $c$ is the speed of light in the tissue determined by $n$,

- $\mu_{t}=\mu_{a}+\mu_{s}$

- $\quad P\left(\hat{s}^{\prime}, \hat{s}\right)$ is the phase function representing the probability of light propagation direction $\hat{s}^{\prime}$

- being scattered into solid angle $\Omega$ around $\widehat{s}$

- $S(\vec{r}, \hat{s}, t)$ describes the light source

RTE includes six independent variables to define radiance at every spatial and temporal point, this makes it very complicated to be solved; by making appropriate assumptions based on the properties of a highly scattering medium the number of variables could be reduced. The main two assumptions are: 1) Scattering is the dominant interaction compared to the absorption hence the radiance would be isotropic after many scattering events; 2) in high scattering medium such as biological tissue the time of the mean free path of the light is significantly shorter than the time for substantial photon fluence change therefore the changes in current density over one mean free path transportation is negligible; this property is called temporal broadening.

Diffusion approximation is only for high scattering medium in which the scattering coefficient is much larger than absorption coefficient; in addition the minimum thickness 
of the sample must be several times greater than photon mean free path. In order to describe the diffusion of the light by random walk theory random step size of $1 / \mu_{s}^{\prime}$ is defined and $\mu_{s}^{\prime}$ is called reduced scattering coefficient. Diffusion approximation depends on reduced scattering coefficient instead of scattering coefficient.

By taking into account the two mentioned assumptions, the RTE is reduced to diffusion equation (approximation):

$\frac{1}{c} \frac{\partial \Phi(\vec{r}, t)}{\partial t}+\mu_{a} \Phi(\vec{r}, t)-\nabla \cdot(D \nabla \Phi(\vec{r}, t))=S(\vec{r}, t) \quad[E q .1-10] \quad$ Diffusion Equation where:

$D=\frac{1}{3\left(\mu_{a}+\mu_{s^{\prime}}\right)}$ is the diffusion coefficient

$\mu_{s^{\prime}}=(1-g) \mu_{s}$ is the reduced scattering coefficient

$g=\cos \theta$ is the scattering anisotropy, $\theta$ is the mean scattering angle in the tissue; $g$ is around 0.9 in biological tissue

\subsubsection{Diffusion equation for a semi-infinite medium; Extrapolated boundary and image source assumptions}

In most of the medical applications of NIRS the source and detector are placed on the surface of the tissue; it is evident that an infinite medium scheme does not model this configurations correctly, therefore a homogeneous semi-infinite medium seems to be more appropriate for modeling absorption with the diffusion approximation [25]. In order to derive an analytical solution to the diffusion equation for different configurations of boundaries of tissue and light source, an appropriate boundary and source condition should be applied. In order to model the fluence the desired boundary is where the fluence is zero however at the physical boundary due to reflectance and backscattering the fluence rate is not zero therefore an extrapolated boundary is assumed at $-z_{b}$ $\left(z_{b}=2 D\right)$ beyond the physical boundary [26]. In addition the diffusion equation requires 
an isotropic source, the NIRS source is not isotropic at the physical surface and is indeed several pencil beams normally incident on a semi-infinite medium though; In order to meet this condition an imaginary source is assumed at a depth at which all photons would be scattered at $z_{0}\left(z_{0}=3 D \approx 1 / \mu_{s}^{\prime}\right)$ and the optical properties of the points located at the depths shallower than $z_{0}$ may not be measured using diffusion approximation [27]. Fig. 1-5 shows the diagram of the extrapolated boundary and initial scattering depth as the imaginary isotropic point source with respect to the physical boundary in a highly scattering medium.

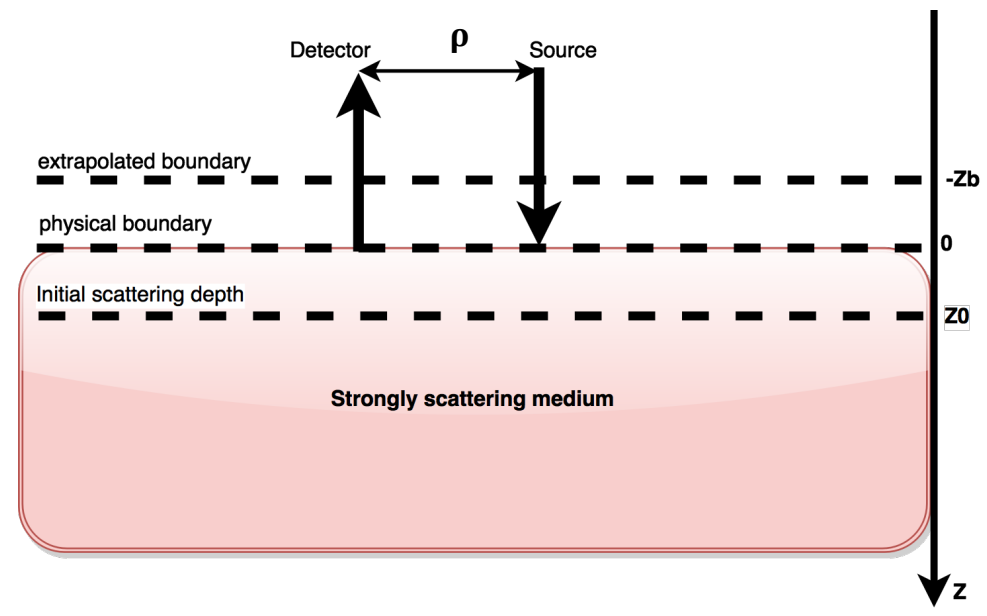

Figure1-5; Schematics of the extrapolated boundary at $-z_{b}$ and imaginary source at $z_{0}$ conditions in a highly scattering medium to meet the diffusion approximation requirements

\subsubsection{Analytical solution to the diffusion equation}

For a semi-infinite medium an analytical solution to the diffusion equation could be obtained considering boundary and image source assumptions. Given the imaginary isotropic point source at $Z_{0}$ (as shown in Fig. 1-5), an image source at reflection of the imaginary source about the plane of the extrapolated zero boundary, the solution to the 
diffusion approximation for a semi-infinite medium with a collimated source on the real boundary is [28] [25]:

$$
\psi=\frac{2}{(4 \pi)^{2}} \frac{S}{D} \frac{\exp \left[-\rho\left(\frac{\mu_{a}}{D}\right)^{1 / 2}\right]}{\rho^{3}}\left[1+\rho\left(\frac{\mu_{a}}{D}\right)^{1 / 2}\right]\left(z_{0}+z_{b}\right) \times\left[z_{b}+3 D\left[1-\frac{\left(z_{0}+z_{b}\right)^{2}+3 z_{b}^{2}}{2 \rho^{2}}\left\{3+\frac{\rho^{2} \frac{\mu_{a}}{D}}{1+\rho\left(\frac{\mu_{a}}{D}\right)^{1 / 2}}\right]\right]\right]
$$

[Eq.1 - 11] Analytical solution to the diffusion equation for a semi-infinite medium

Fantini et al. have derived this solution and confirmed it with the Monte Carlo simulation results [25].

Using this solution the fluence rate at every time point is calculated for each wavelength.

This model is based on $\mu_{a}$ and $\mu_{s^{\prime}}$. By definition we have:

$$
\begin{aligned}
& \mu_{a}=\left[\mathrm{HbO}_{2}\right] \varepsilon(\lambda)_{\mathrm{HbO} 2}+[\mathrm{HHb}] \varepsilon(\lambda)_{H H b}+[\mathrm{Cyt}-\mathrm{ox}] \varepsilon(\lambda)_{C \mathrm{CO}} \quad[\text { Eq. 1 - 12] } \\
& \mu_{s}{ }^{\prime}(\lambda)=\mu_{s}{ }^{\prime}{ }_{800}\left(\frac{\lambda}{800}\right)^{-\alpha}[\boldsymbol{E q} \mathbf{1}-\mathbf{1 3}]
\end{aligned}
$$

$\alpha$ and $\mu_{s 800}^{\prime}$ have been measured experimentally for different types of tissues in several studies by V. Toronov et al. [29] and P. Zee [30]. $\mu_{s}^{\prime} 800$ is the reduced scattering coefficient at isobestic point of biological tissue. Reduced scattering coefficient is independent of the chromophore's concentrations and is calculated once for every wavelength and it is assumed that the it's changes are negligible during the experiment.

Using equation 1-11, the fluence is calculated at every time point for all wavelengths. In the next step measured absorbance is quantified using equation 1-1, finally concentration changes are calculated at different time points. In multi wavelength NIRS same as what was explained in MBLL approach a system of equations is solved to calculate concentration changes; in hyperspectral approach for the reasons that were explained 
previously, concentration changes are calculated by performing a non-linear least square fitting between the absorbance changes and fluence changes:

$$
\ln \frac{\psi_{\lambda}\left(\mu_{a}+\Delta \mu_{a}, \mu_{s}^{\prime}\right)}{\psi_{\lambda}\left(\mu_{a}, \mu_{s}^{\prime}\right)} \stackrel{\text { non-linear-fit }}{\longrightarrow} \ln \left[\frac{I(\lambda)}{I_{0}(\lambda)}\right]
$$

The least-square curve fit gives us the $\Delta \mu_{a}$; from equation 1-12 we have:

$$
\Delta \mu_{a}=\sum \Delta[C] \varepsilon(\lambda) \quad[E \boldsymbol{q} .1-13]
$$

Concentration changes are calculated for different chromophores at different time points just by deviding $\Delta \mu_{a}$ by the molar extinction coefficient of each chromophore for every wavelength.

One of the advantages of employing the diffusion equation for modeling the absorbance is that this model is based on reduced scattering coefficient which defines the mean free pathlength of the light and independent of the travellig distance of the light as opposed to the MBLL that needs DPF for calculation and DPF can not be measured directly with CW NIRS data. In addition due to the cross-talk effect in MBLL and underestimation systematic error of that as reported by L kocsis et al. [23] and also as my research results has shown, the diffusion approximation works much more effectively than the MBLL specially for low concentration chromophores such as Cyt-ox.

\subsection{Hyperspectral NIRS signal processing algorithms}

Data fitting is the most common approach for deconvolution of different chromophores from hyperspectral NIRS data. Quality of the data fitting is very important mostly for deconvolution of the Cyt-ox redox state which has relatively lower concentration than oxy and deoxy hemoglobin and could be easily contaminated by the physiological or 
instrumental noise or the cross-talk with hemoglobin hence in order to increase the quality of the fitting several algorithms has been developed.

In this thesis I have designed two hyperspectral NIRS studies, one functional and one non-functional; in the first set of experiments I performed an event related functional broadband NIRS and different algorithms were assessed for deconvolution of the chromophores. In this group the brain activity of sixteen participants have been monitored during a driving test; an event-related scenario was designed that included different driving tasks and distraction during driving. Fig. 1-6 shows different examined algorithms for out functional NIRS data. In addition to comparing MBLL with diffusion approximation techniques, application of the "independent component analysis" (ICA) in denoising the signal for extraction of Cyt-ox changes have been assessed. ICA principals are explained in the next section. In functional NIRS studies we usually expect very small changes in Cyt-ox redox state that could be easily contaminated with the physiological noise, motion artifacts and cross-talk with $\mathrm{HbO}_{2}$ and $\mathrm{HHb}$ signal. The effectiveness of the isolation of different chromophores has been assessed through statistical analysis (ANOVA and t-test) of the group results and the conclusion has been made based on the best obtained statistical significance by different processing approaches. According to the statistical analysis results the diffusion approximation along with ICA resulted in the best isolation of chromophores that was very consistent among all participants. 


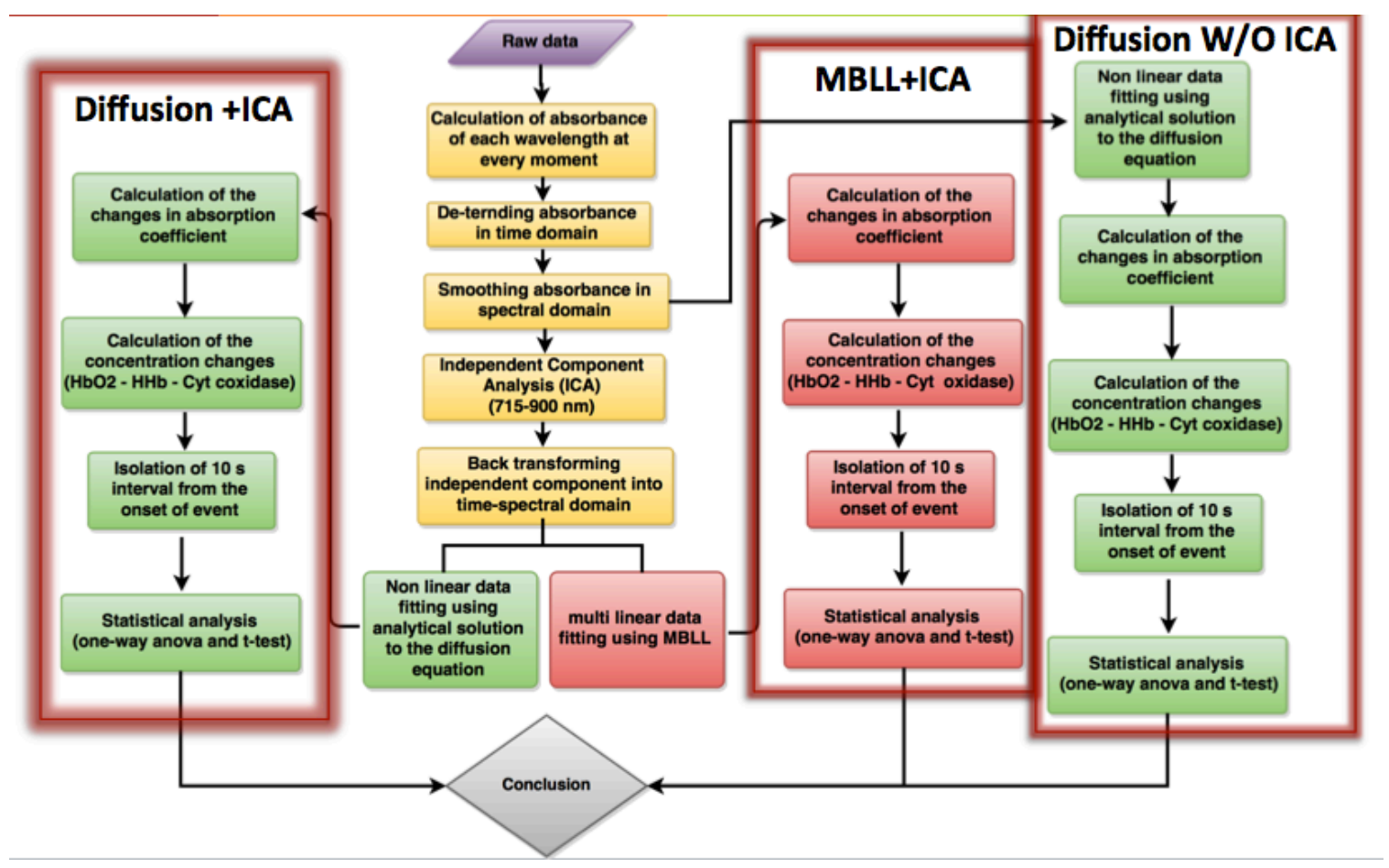

Figure 1-6; hyperspectral functional NIRS data processing algorithm

In the second set of experiments (non-functional studies) we monitored brain activity and metabolism level during cardiac arrest and resuscitation. Eighteen pigs were examined and NIRS signal along with hemodynamics (blood pressure and blood flow) were measured at different tissues (muscle and brain). During the cardiac arrest and resuscitation large changes are induced on all chromophores thus the probability of contamination of the signals with other factors is minimal. Figure 1-7 shows the signal processing algorithms that were assesses for deconvolution of different chromophores. We have assessed both MBLL and diffusion approximation techniques. A novel approach for stepwise deconvolution of different chromophores has been introduced that resulted in the best isolation of chromophores. 


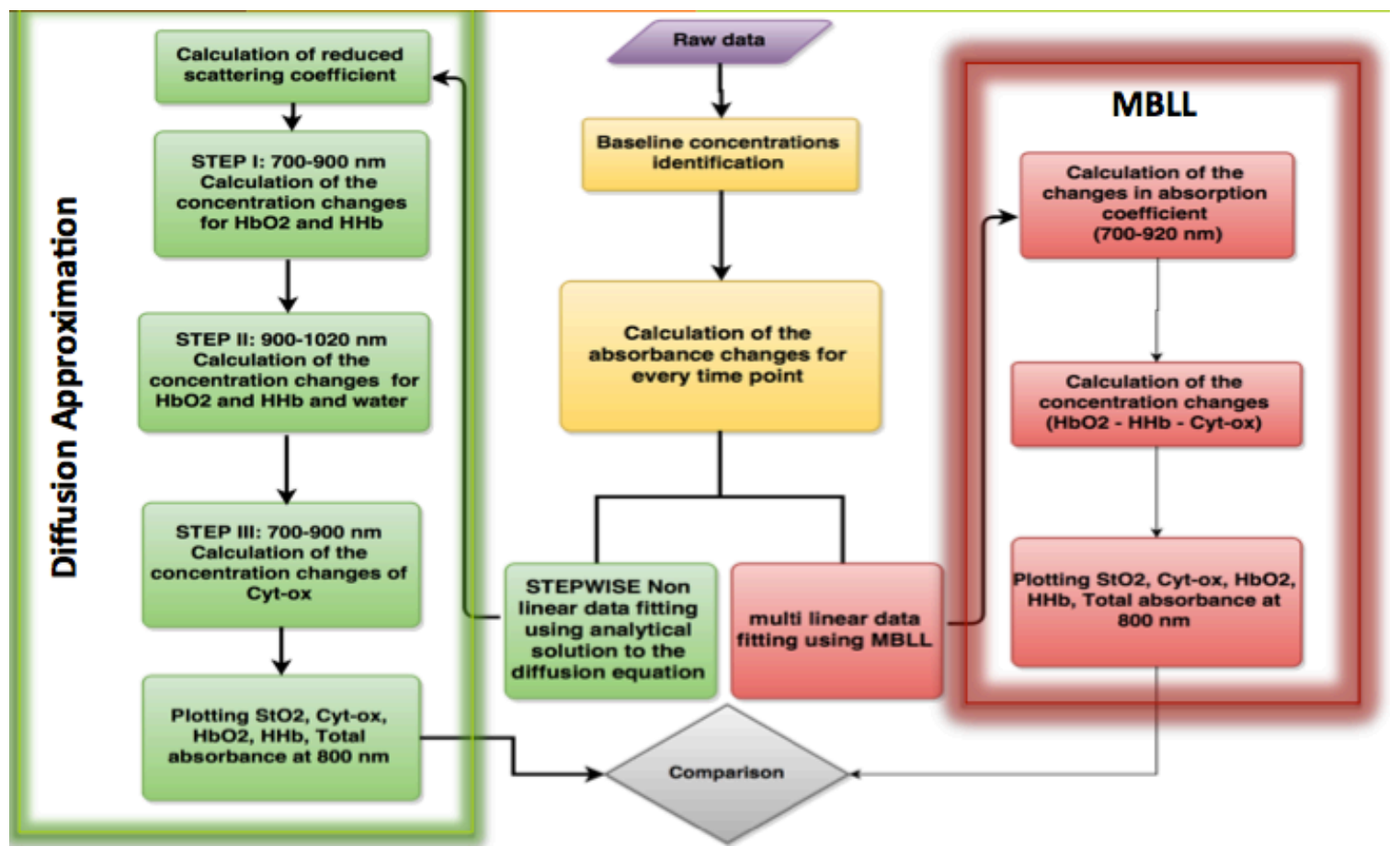

Figure 1-7; hyperspectral non-functional NIRS data processing algorithms

\subsubsection{Independent Component Analysis (ICA) for hNIRS}

Resolving the functional signal from contaminations from motion artifacts, physiological noise and cross-talk of different chromophores is one of the main challenges in functional NIRS. Toronov et al [31] introduced ICA application for calculation of $\mathrm{HbO}_{2}$ and $\mathrm{HHb}$. In this thesis effectiveness of ICA in deconvolution of Cyt-ox changes has been investigated.

Independent component analysis (ICA) is a statistical tool that separates a multivariate signal to its subcomponents. ICA is one of the methods for blind source separation. [32] [33]. Blind source deconvolution is separation of a set of source signals from mixed signals without any information bout the source or mixing signals. Basically blind separation tries to narrow the number of solutions in a way that desired solutions remain intact. One approach to blindly separate sources is to seek for solutions that are minimally 
correlated or maximally independent based on statistical analysis; this is called independent component analysis or ICA.

Suppose we are measuring brain NIRS signal. The recorded signal is mainly generated simultaneously by $\mathrm{HbO}_{2}, \mathrm{HHb}$, Cyt-ox, water and other chromophores, however instrumental noise, motion artifacts, physiological noise are contaminating the original signal; this is called cocktail-part problem [32]. Let's assume the NIRS measured signal corresponding to $\mathrm{HbO}_{2}$ and $\mathrm{HHb}$ is denoted by $x_{1}(t)$ and $x_{2}(t)$, with $x_{1}$ and $x_{2}$ are the signal the amplitudes, and t represents the the time point. Each of these recorded signals is a weighted sum of the signals generated by the two chromophores, which we denote by $s_{1}(t)$ and $s_{1}(t)$. We could express this as a system of equations:

$x_{1}(t)=a_{11} s_{1}+a_{12} s_{2} \quad[$ Eq. $1-15]$

$x_{2}(t)=a_{21} s_{1}+a_{22} s_{2}$

To solve the above system equations we need to estimate $a_{i j}$. To estimate the $a_{i j}$ one approach (regardless of any information about the signal sources) is to take advantage of some information on the statistical properties of the signal sources $s_{i}(t)$. Surprisingly it is sufficient to assume that the signal sources: $s_{1}(t), s_{2}(t), \ldots, s_{n}(t)$ at each time point are statistically independent [32] [33]. The independency assumption does need not be 100\% true in practice. ICA, can be used to estimate the $a_{i j}$ based on the their independence assumption, which allows us to separate the original source signals $s_{1}(t), s_{2}(t), \ldots, s_{n}(t)$ from their measured mixtures $x_{1}(t), x_{2}(t), \ldots, x_{n}(t)$.

Two variables are called statistically independent if information on one variable does not give any information on other variable, and vice versa $[33]$. Indeed In probability theory, 
when two events are independent it means that the occurrence of one event has no effect on the probability that the other occurs.

Independence of two variables can be defined by their joint and conditional probability density function (PDF) meaning that two variables $\mathrm{x}$ and $\mathrm{y}$ are independent if and only if their joint PDF is equal to the product of their individual PDF:

$P_{y}(y / x)=\frac{P_{x, y}(x, y)}{P_{x}(x)}=P_{y}(y) \Leftrightarrow P_{x, y}(x, y)=P_{x}(X) P_{y}(Y) \quad[$ Eq. 1 - 14 $]$

The two variables are said to be uncorrelated when their covariance is zero; when two variables are independent, they are definitely uncorrelated but uncorrelated variables are not necessarily independent [34].

ICA application requires two conditions: 1) the source signals must be independent of each other; 2) the values corresponding to the source signals should have non-Gaussian distribution [32]. Gaussian sources are eliminated by ICA because their joint PDF shows completely symmetric density and does not follow the relationship shown by Eq. 1-14.

B. Ans, J. Herault and C. Jutten introduced ICA framework in 1985 [35] but it was most clearly stated by Pierre Comon in 1994 [36]. There are many algorithms in the literatures that implement ICA. One of the most popular algorithms that have been implemented in this thesis as well, is FastICA introduced by T. Bell and T. Sejnowski in 1995 [37].

Some data preprocessing steps are useful before doing ICA to simplify and reduce the complexity of the problem. Preprocessing steps include: centering, whitening and other steps depending on the application itself such as reducing the dimensions of the data. Centering steps subtracts the mean of the data from all data points thus the data will be centered on zero. Whitening the signal removes the linear dependencies and normalized projection variance [38]. Reducing the dimensions of the signal in cases such as 
hyperspectral NIRS that employs more than 1000 wavelengths and each wavelength is a signal source, is very useful and could simplify the ICA process.

\subsubsection{Stepwise algorithm for deconvolution of tissue chromophores}

In order to better resolve Cyt-ox changes different techniques have been applied. C. Cooper et al. in 1999 [39] showed that Cyt-ox signal is critically dependent on the wavelength range. H. Heekeren et al. in 1999 [40] applied an algorithm that performed the least-square fitting procedure to different spectral ranges and they found the best range that gives the most statistically reliable results. In their approach all chromophores were resolved at each examined range.

We have introduced a novel hNIRS signal processing algorithm shown in Fig. 1-7, for hyperspectral NIRS data fitting employing a stepwise fitting procedure that deconvolve different chromophores in different steps within different spectral bands.

The main goal of this algorithm is to improve deconvolving Cyt-ox signal from all physiological or instrumental noise and cross talk with high concentraton chromophores. In this algorithm $\mathrm{HbO}_{2}$ and $\mathrm{HHb}$ were initially isolated within spectral range of 700-900 $\mathrm{nm}$. Although in NIR region the contribution of the water to the NIRS signal is minor, to better isolate Cyt-ox signal, water concentration changes was calculated within spectral band of 900-1020 nm, in this step the calculated values of $\mathrm{HbO}_{2}$ and $\mathrm{HHb}$ in the first step were fed into the second fitting process. In the final step all the calculated concentrations in the first two steps were fed into the fitting process in the range of 700-800 nm.

As shown in Fig. 1-8, before 700nm melanin is the dominant NIR absorber and after 900 $\mathrm{nm}$ water dominates $\mathrm{HbO}_{2}$ hence $700-900 \mathrm{~nm}$ is the best spectral band for $\mathrm{HbO}_{2}$ and $\mathrm{HHb}$. 
The spectral band at which the Cyt-ox (oxidized minus reduced) has its maximum extinction coefficient and is minimally correlated with $\mathrm{HbO}_{2}$ and $\mathrm{HHb}$ is $780-900 \mathrm{~nm}$. In our algorithm we used the first derivative of the extinction coefficient of the Cyt-ox redox changes therefore $700-800 \mathrm{~nm}$ at which maximum changes are observed in the first order derivative, was selected for the fitting process. The usefullness of applying the derivatives of the extinction coefficient's spectra was introduced by Toronov et al in 2012 [41].

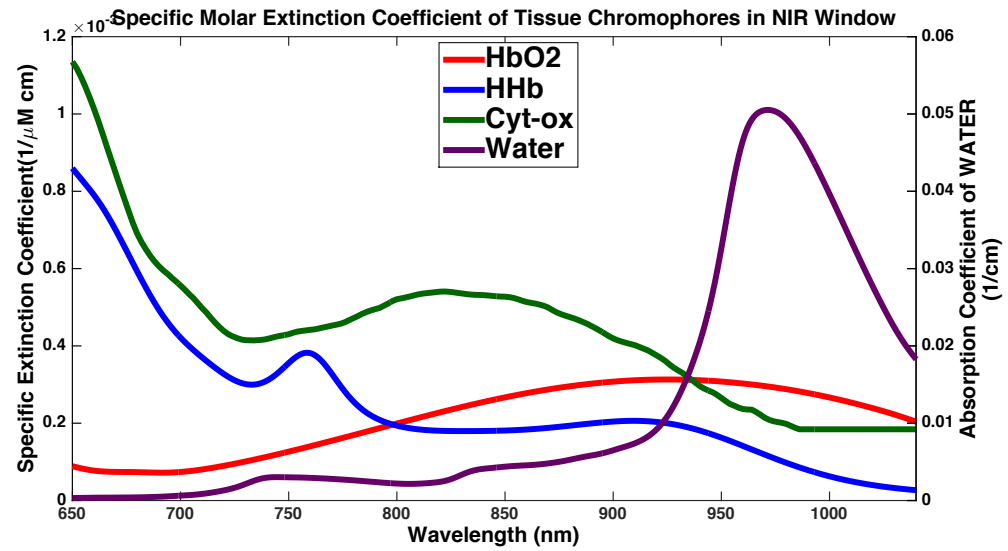

Figure 1-8, Extinction spectra of main chromophoes in NIR region of the EM spectrum

\subsection{Physiological interpretation of NIRS results}

NIRS enables us to measure different tissue chromophores non-invasively. $\mathrm{HbO}_{2}$ and $\mathrm{HHb}$ are the dominant NIR absorbers within applied spectral range. Suppose a NIRS measurement on the brain; $\mathrm{HbO}_{2}$ enhancement represents increasing regional oxygen delivery to the cerebral tissue. Neural activation is accompanied by increased regional cerebral blood flow (rCBF); this is though to be coupled with enhancement of cerebral blood volume through vasodilation of already perfused vessels [42] or by partial volumetric expansion of the vessels so called recruitment [43]. 
Summation of the NIRS measured changes in $\mathrm{HbO}_{2}$ and $\mathrm{HHb}$ reflects the changes in blood volume in the illuminated area [44].

It is widely accepted that the $\mathrm{rCBF}$ gain exceeds the increases in the regional cerebral oxygen consumption rate [45], this results in a decrease in $\mathrm{HHb}$ in venous blood while $\mathrm{HbO}_{2}$ supply has increased. Therefore increases in $\mathrm{HbO}_{2}$ with a decrease in $\mathrm{HHb}$ are believed to indicate regional brain activation in NIRS measurement. In NIRS measurements $\mathrm{HbO}_{2}$ seems to be the most sensitive indicator of changes in $\mathrm{rCBF}$ [44]. As it was explained in the previous sections Cyt-ox is responsible for $95 \%$ of intracellular oxygen metabolism. The measured difference between oxidized and reduced enzyme could reflect the level of oxygen metabolism and ATP synthesis. Changes in oxygen delivery represented by $\mathrm{HbO}_{2}$ may not be always accompanied by enhancement of metabolism and energy synthesis especially when cells are diseased or dead; therefore measurement of Cyt-ox changes could be very important index as it reflects intracellular oxygen metabolism level and provides higher brain specificity than hemoglobin [46]. In healthy brain it is expected to observe enhanced $\mathrm{HbO}_{2}$ and Cyt-ox in activated regions of the tissue and vise versa. 


\title{
Chapter 2 \\ Journal manuscript 1
}

\section{Event-related Changes of the Prefrontal Cortex Activity During Driving Measured by Hyperspectral fNIRS Reyhaneh Nosrati*, Kristin Vesely, Tom A. Schweizer, Vlad Toronov}

\author{
Author Affiliations \\ Reyhaneh Nosrati \\ Department of Biomedical Physics, Ryerson University, 350 Victoria Street, Toronto, ON, Canada, M5B 2K3, \\ Reyhaneh.nosrati@ryerson.ca \\ Kristin Vesely \\ Keenan Research Centre of the Li Ka Shing Knowledge Institute of St. Michael's Hospital, 30 Bond Street, \\ Toronto, ON, Canada, M5B 1W8, kristin.vesely@mail.utoronto.ca
}

Tom A. Schweizer

Keenan Research Centre of the Li Ka Shing Knowledge Institute of St. Michael's Hospital, 30 Bond Street, Toronto, 0N, Canada, M5B 1W8, SchweizerT@smh.ca

Department of Surgery, Faculty of Medicine (Neurosurgery), University of Toronto, Toronto, ON, Canada Institute of Biomaterials and Biomedical Engineering, University of Toronto, Toronto, ON, Canada

\section{Vlad Toronov}

Department of Biomedical Physics, Ryerson University, 350 Victoria Street, Toronto, ON, Canada, M5B 2K3, toronov@ryerson.ca

Keywords:Hyperspectral functional near infrared spectroscopy (h-fNIRS), Functional magnetic resonance imaging (fMRI), Driving simulation, Prefrontal cortex (PFC), Distracted driving condition, Cognitive tasks 


\subsection{Abstract}

Driving requires visual attention and involves mostly posterior brain regions. A recent fMRI study during simulated driving has shown that complex driving task such as right turn and left turn in traffic activate the posterior cortex and deactivate bilateral prefrontal cortices, the prefrontal cortices become significantly activated due to distractions during driving such as answering simple questions irrelevant to driving (Schweizer TA, 2013). Since MRI scanning includes specific noise, supine position, and other unusual conditions, these and other important issues of brain activity related to driving need to be ultimately investigated during actual driving. In order to check if such a realistic investigation is possible we measured the concentration changes of concentrations of oxygenated $\left(\Delta \mathrm{HbO}_{2}\right)$ and deoxygenated $(\Delta \mathrm{HHb})$ hemoglobin and of the oxidized state of cytochrome $\mathrm{c}$ oxidase $(\triangle \mathrm{CCO})$, on the right and left prefrontal cortices (PFC) simultaneously during simulated driving on sixteen healthy right-handed participants (aged between $22-32$ ). We used our in house hyperspectral functional near infrared spectroscopy system based on a portable spectrometer with cooled CCD detector and a driving simulator with a fully functional steering wheel and foot pedals. Each participant performed different driving tasks and participants were distracted during some driving conditions by asking general knowledge true/false questions. Our findings suggest that complex driving task (non-distracted) deactivate PFC while distractions during driving significantly activate PFC, which is in agreement with the previous fMRI results (Schweizer TA, 2013). Also we found the behavior of $\Delta$ ox-CCO to be very consistent with that of $\Delta \mathrm{HbO}_{2}$ and $\Delta \mathrm{HHb}$. Overall we conclude that fNIRS can be used to study brain activity during actual driving. 


\subsection{Introduction}

Driving is an activity that involves various functions of the central nervous system (CNS) such as visual attention, planning, decision-making and motor control; each of these functions activate specific regions of the CNS. For instance visual attention activates occipital lobe (posterior brain network), motor control activates the parietal lobe of the brain and also the spinal cord and more complex driving tasks that require thinking, planning or decision making mostly activate the frontal lobes. Malfunction of any of these regions may increase the risk of vehicle crashes. Activation of brain regions could be assessed by measuring the concentration changes of some chromophores such as oxygenated hemoglobin $\left(\mathrm{HbO}_{2}\right)$, deoxygenated hemoglobin $(\mathrm{HHb})$ and an intracellular metabolism marker (mitochondrial membrane enzyme) cytochrome c oxidase (CCO) (Banaji, 2006).

Near infrared spectroscopy (NIRS) is a portable non-invasive method for real-time measurement of brain activity in real-life situations without noise and movement limitations or interfering with biological systems (Gérard Derosière, 2013). NIRS monitors the concentration changes of tissue chromophores based on changes in the attenuation of near-infrared spectra. $\mathrm{HbO}_{2}, \mathrm{HHb}$ and $\mathrm{CCO}$ are significant NIR absorbers (Jobsis, 1977). Oxygenated and deoxybgenated hemoglobin reflect intravascular oxygenation. $\mathrm{CCO}$ is the terminal electron acceptor in mitochondrial respiratory chain (Richter, 2003) and contains four redox active metal centers; the copper $\mathrm{A}\left(\mathrm{Cu}_{\mathrm{A}}\right)$ center has a distinct redox-sensitive absorbance band in NIR region of the electromagnetic spectrum; it is responsible for $95 \%$ of cellular oxygen metabolism and ATP synthesis (Jobsis, 1977). Total concentration of CCO in the short term is almost constant and it 
interconverts between reduced and oxidized states. The concentration changes of oxidized $\mathrm{CCO}(\triangle \mathrm{CCO})$ that can be monitored by NIRS, represent changes in the $\mathrm{CCO}$ redox state and reflects the balance between cerebral oxygen delivery and utilization (Smith, 2011; Christina Kolyva, 2014). The brain cells have the highest mitochondrial density among all other body cells thus the concentration of $\mathrm{CCO}$ is significantly higher and easier to measure within brain tissue.

Hyper-spectral NIRS provides both high temporal resolution (in order of millisecond) and also best possible spectral resolution that improves the quality of isolation of different chromophores having overlapping absorption spectra. In addition, the hyperspectral fNIRS allows for highly efficient noise reduction using independent component analysis (Schelkanova, 2012)

In this study we aimed to investigate how the healthy adult brain responds to complex driving tasks using dual channel hyperspectral fNIRS. It has been shown that in complex motor and cognitive tasks the prefrontal cortex (PFC) is highly involved (Earl K. Miller, 2001) in addition this hairless area of the scalp minimizes the scattering and attenuation effect of the hairs. In our measurements optodes were placed on the right and left PFC to measure $\Delta \mathrm{HbO}_{2}, \Delta \mathrm{HHb}$ and $\Delta \mathrm{ox}-\mathrm{CCO}$ during driving and cranial activity of the PFC in complex driving tasks was assessed.

Recently a similar study has been done with the same driving simulation system and scenarios monitoring brain activity using fMRI (Schweizer TA, 2013). In this study Schweizer and colleagues reported that normally complex driving task that require higher visual attention activated occipital lobe and deactivated frontal lobe, however distraction during driving shifted the brain activation from posterior to prefrontal cortex. 
Another study during actual driving has been done using fNIRS assessing the brain activity during acceleration and deceleration in day and night (Yoshino K O. N., 2013). They have shown that the frontal eye field (FEF) of the prefrontal cortex is highly activated while the speed is changing. However due to safety issues, those experiments were performed on an unopened expressway and the only stimulations were acceleration and deceleration.

\subsection{Materials and Methods}

\subsubsection{Subjects}

Sixteen healthy right-handed adult subjects, aged between 20 and 32 (Mean $=24.19$, SD $=3.76)$ participated in this study (5 males and 11 females). All subjects had normal or corrected to normal vision and provided written informed consent prior to beginning of the study. All subjects had driver's license with average driving experience of 6.38 years $(\mathrm{SD}=4.26)$. The human subject research protocol REB 2008-003 was approved by the Ryerson University Ethics Board.

\subsubsection{Driving simulation system}

The driving simulation set up contained a fully functional steering wheel, brake and accelerator pedals, an LCD monitor and speakers (Figure 2-1-a). STISIM drive software (version 2.08.08, System Technology Inc., Hawthorne, CA) was used to introduce driving tasks or distraction at proper moments and also record driving parameters such as speed, direction, acceleration and timing. In addition to the road and traffic signs participants could see the rear-view mirror, speedometer, RPM gauge and turn signals. On the left and right side of the steering wheel there were two buttons and participants were asked to 
press the right one for TRUE and the left one for FALSE to answer general knowledge questions while driving. Participants sat on a chair in front of the monitor and steering wheel and were asked to drive safely, follow the traffic rules and GPS commands. All participants underwent a 10 minutes training session prior to the NIRS to get familiar with the system.
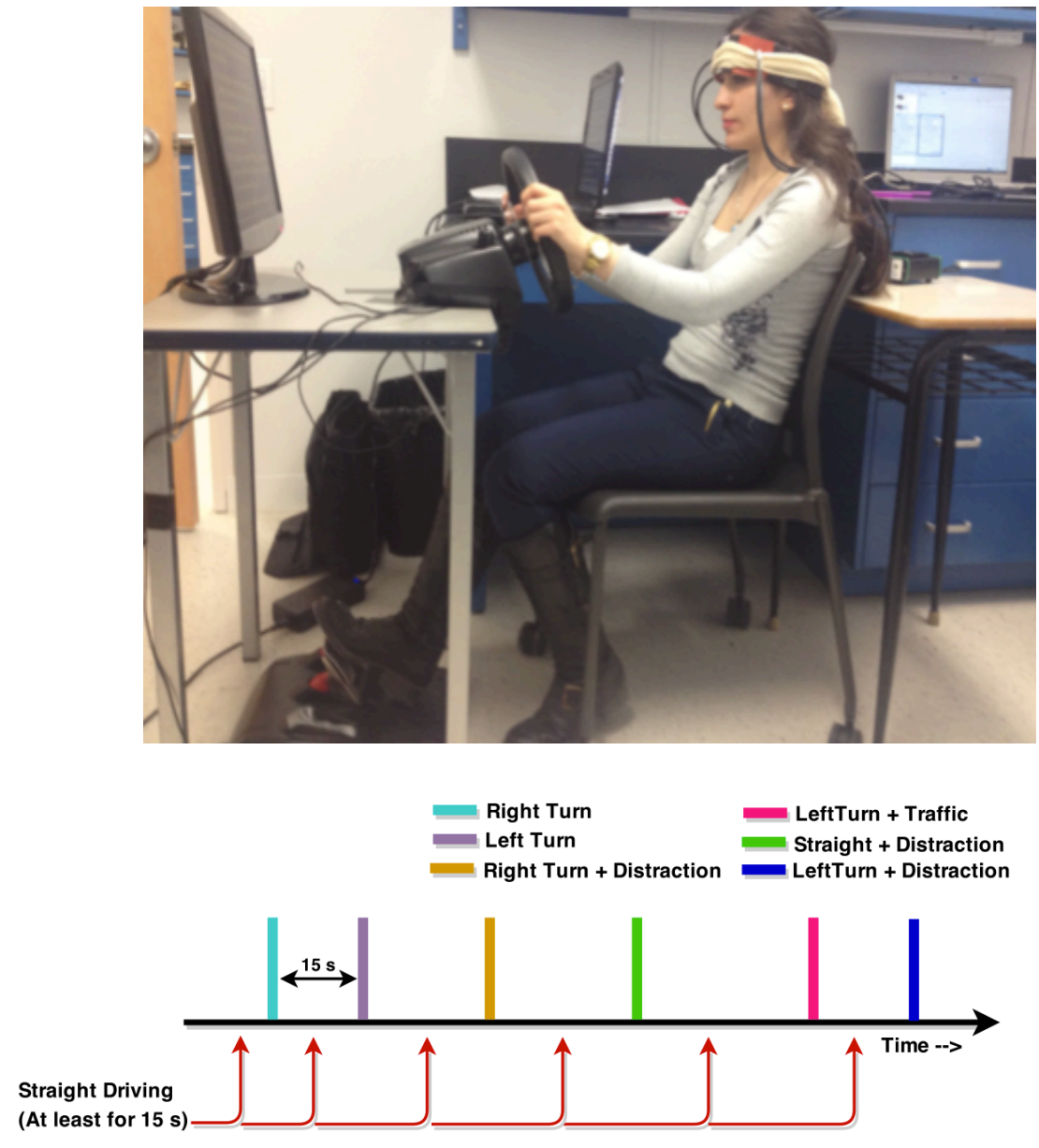

Figure 2-1-a) Driving simulator and fNIRS measurement apparatus; b) Event-related driving scenario (second driving scenario including distraction) 


\subsubsection{Driving scenarios}

With each subject fNIRS was performed during two driving scenarios: one without distractions and another with distractions (figure 2-1-b). Each of two blocks took approximately 10 minutes (depending on the speed of driving). Both driving scenarios included several routine driving tasks such as right turns and left turns and the turns combined with the presence of pedestrians or traffic at intersections. There were about at least 10s of straight driving between Aall driving tasks were separated by at least 10s of straight driving. Each task was introduced by a recorded voice (similar to GPS commands) 5 seconds prior to the task (e.g. "Turn left at the traffic light"). In the second scenario participants were distracted during right turn and straight driving left turn by general knowledge true/false questions (e.g. "A horse is greater than a puppy"), which they answered by pressing $\mathrm{T}$ or F buttons placed on the steering wheel. Driving tasks that were isolated and analyzed contained: straight driving, distracted straight driving, accident avoidance, left turn in traffic, distracted left turn in traffic, right turn and right turn with distraction.

\subsection{4 fNIRS system and procedures}

A single halogen lamp source along with two QE65000 Ocean Optics spectrometers were used to obtain measurements from right and left prefrontal cortex (PFC) with $1 \mathrm{~Hz}$ sampling frequency. One meter long optical fiber bundles with $3 \mathrm{~mm}$ core diameter were use to transmit the light from the broadband source to the brain and also to transmit the detected light to the broadband spectrometers. The positions of the optodes were determined using 10/20 EEG system (using an EEG standard cap). F7 and F8 locations were marked on each participant and optodes were placed on those locations with $30 \mathrm{~mm}$ 
source-detector distance. The equipment irradiated and detected broadband wavelength range of $6500-1100 \mathrm{~nm}$ on right and left PFC simultaneously. fNIRS optodes were perpendicularly placed on marked locations on the forehead using a $2 \mathrm{~cm}$ thick headband and an elastic bandage was used to fix their positions. fNIRS devices were securely placed on a table behind the subject to minimize the interference between driver's movements and field of view with the fibers.

\subsubsection{Data analysis}

\subsubsection{1 fNIRS algorithm}

Data analysis was performed using MATLAB (Mathworks, MA, version R2013b). The signal preprocessing included steps as described in (Schelkanova, 2012): de-trending and de-noising of the absorbance changes using the Matlab independent component analysis algorithm FastICA (Gävert, 2005) within the wavelength range of $715-900 \mathrm{~nm}$. The number of independent components was reduced to five. These remaining five independent components were back-transformed to the original time-spectral domain using the inverse of the mixing matrix obtained using FastICA (Schelkanova, 2012). All these steps were taken to remove the baseline and to de-noise the absorbance changes. In the next step the measured absorbance changes was non-linearly fitted to the modeled flux changes calculated by the analytical solution to the diffusion equation for the a semiinfinite medium. The non-linear fit was performed using the Matlab function 'Isqcurvefit', where the parameters of the fit were $\Delta\left[\mathrm{HbO}_{2}\right], \Delta[\mathrm{HHb}]$ and $\Delta[\mathrm{ox}-\mathrm{CCO}]$. After the non-linear fit the changes in absorption coefficient is estimated and eventually changes in concentration of different chromophores is calculated.

$\mu_{a}=\left[\mathrm{HbO}_{2}\right] \varepsilon(\lambda)_{\mathrm{HbO} 2}+[\mathrm{HHb}] \varepsilon(\lambda)_{\mathrm{HHb}}+[\mathrm{ox}-\mathrm{CCO}] \varepsilon(\lambda)_{C \mathrm{CO}}$ 
$\ln \frac{\psi_{\lambda}\left(\mu_{a}+\Delta \mu_{a}, \mu_{s}^{\prime}, t\right)}{\psi_{\lambda}\left(\mu_{a}, \mu_{s}^{\prime}, t_{0}\right)} \stackrel{\text { non-linear-fit }}{\longrightarrow} \ln \left[\frac{\operatorname{Raw} \operatorname{Data}(\lambda, t)}{\text { Reference Data }\left(\lambda, t_{0}\right)}\right]$

The baseline values of the absorption coefficient were computed assuming the baseline values $\left[\mathrm{HbO}_{2}\right]=60 \mu \mathrm{M},[\mathrm{HHb}]=20 \mu \mathrm{M}$ and the water fraction of $85 \%$ (Yeganeh Hadi Zabihi, 2012). As we only consider the concentration changes tThe reduced scattering coefficient was assumed to be constant and it was calculated as function of the wavelength was modeled using using the power law shown by the following equation. The detailed method of calculation is described in (Yeganeh Hadi Zabihi, 2012).

$\mu_{s}^{\prime}(\lambda)=\mu_{s 800}^{\prime}\left(\frac{\lambda}{800}\right)^{-a}$

$$
\begin{gathered}
\mu_{s 800}^{\prime} \approx 0.5-1[1 / \mathrm{cm}] \\
a \approx 0.2-5
\end{gathered}
$$

After calculation of the $\mathrm{HbO} 2, \mathrm{HHb}$ and $\mathrm{CCO}$ concentration changes in the time domain, in the last step for every driving task $10 \mathrm{~s}$ interval starting from the onset of the event was isolated for further analysis.

\subsubsection{Statistical analysis}

Five driving tasks (events) were considered for analysis: distracted straight driving, accident avoidance, left turn in traffic, distracted left turn in traffic, right turn and distracted right turn with distraction. For each participant and for each event the changes 
in the chromophore concentrations: $\Delta\left[\mathrm{HbO}_{2}\right], \Delta[\mathrm{HHb}]$ and $\Delta[\mathrm{ox}-\mathrm{CCO}]$ were calculated and isolated within a 10 second time interval right after the onset of each the event.

For each isolated time interval corresponding to the events, the initial value (at the very first moment of the event) was subtracted from the corresponding data trace and the event-related changes from the onset of each event were subject to the group statistical analysis. In addition event-related changes in PFC have been compared between distracted vs. non-distracted conditions to assess how distraction, as a secondary cognitive task, affects activity of the PFC.

Statistical analysis including means, standard deviations, standard errors, pairedsample tT-tests and one-way ANOVA were performed in MATLAB (R2013b version 8.2, Mathworks, USA).

After the isolation of the 10-second time intervals for each of the five event types, all results were averaged over 16 subjects and the standard deviation and standard error were calculated and are shown in the figures as error bars. In order to evaluate statistical significance of the event-related changes, the paired-sample t-test was performed between the very first moment of the stimulation and the moment of the maximum changes in the group-averaged traces (45-810 seconds after the onset of the event). Statistical significance was assumed at $\mathrm{p}<0.05$.

In order to compare the event-related changes between distracted vs. non-distracted driving one-way ANOVA was conducted. The data being compared were put in two groups of "distracted" and "non-distracted" then one-way ANOVA was performed between groups; The "non-distracted" group contained right turn and left turn in traffic. Distracted conditions contained: Distracted right turn and distracted left turn. mean 
Averaged changes in $\Delta\left[\mathrm{HbO}_{2}\right], \Delta[\mathrm{HHb}]$ and $\Delta[\mathrm{ox}-\mathrm{CCO}]$ for each group and $\mathrm{p}$-values for the difference between corresponding means were obtained.

To assess the impact of distraction during different driving task, three distracted conditions including distracted right turn, distracted left turn and distracted straight driving were compared using t-test and maximum induced changes.

\subsection{Results}

\subsection{1 "Right turn" vs. "Right turn + distraction"}

Figure 2-2-a shows the changes during right turn for some random participants. Figure 23-a shows the average traces of $\Delta\left[\mathrm{HbO}_{2}\right], \Delta[\mathrm{HHb}]$ and $\Delta[\mathrm{ox}-\mathrm{CCO}]$ concentration changes of $\mathrm{HbO}_{2}, \mathrm{HHb}$ and ox-CCO under non-distracted and distractedduring right turn in right PFC. During the non-distracted condition $\mathrm{HbO}_{2}$ concentration decreased $(\mathrm{p}<0.05)$ and $\mathrm{HHb}$ increased significantly in both sides $(\mathrm{p}<0.05)$; concentration of ox-CCO decreased in both sides but the changes were significant $(\mathrm{p}<0.05)$ only in the right side.

\subsection{2 "Right turn + distraction"}

Figure 2-2-b shows the changes during distracted right turn for some random participants. Figure 2-3-b shows the average traces of $\Delta\left[\mathrm{HbO}_{2}\right], \Delta[\mathrm{HHb}]$ and $\Delta[\mathrm{ox}-\mathrm{CCO}]$ during distracted right turn in right PFC. In both right and left PFC under distracted condition $\mathrm{HbO}_{2}$ concentration increased ( $\left.\mathrm{p}<0.05\right)$, $\mathrm{HHb}$ decreased but it was statistically significant only in the right PFC $(\mathrm{p}<0.05)$ and also ox-CCO increased however the changes were statistically significant only in the left PFC. 

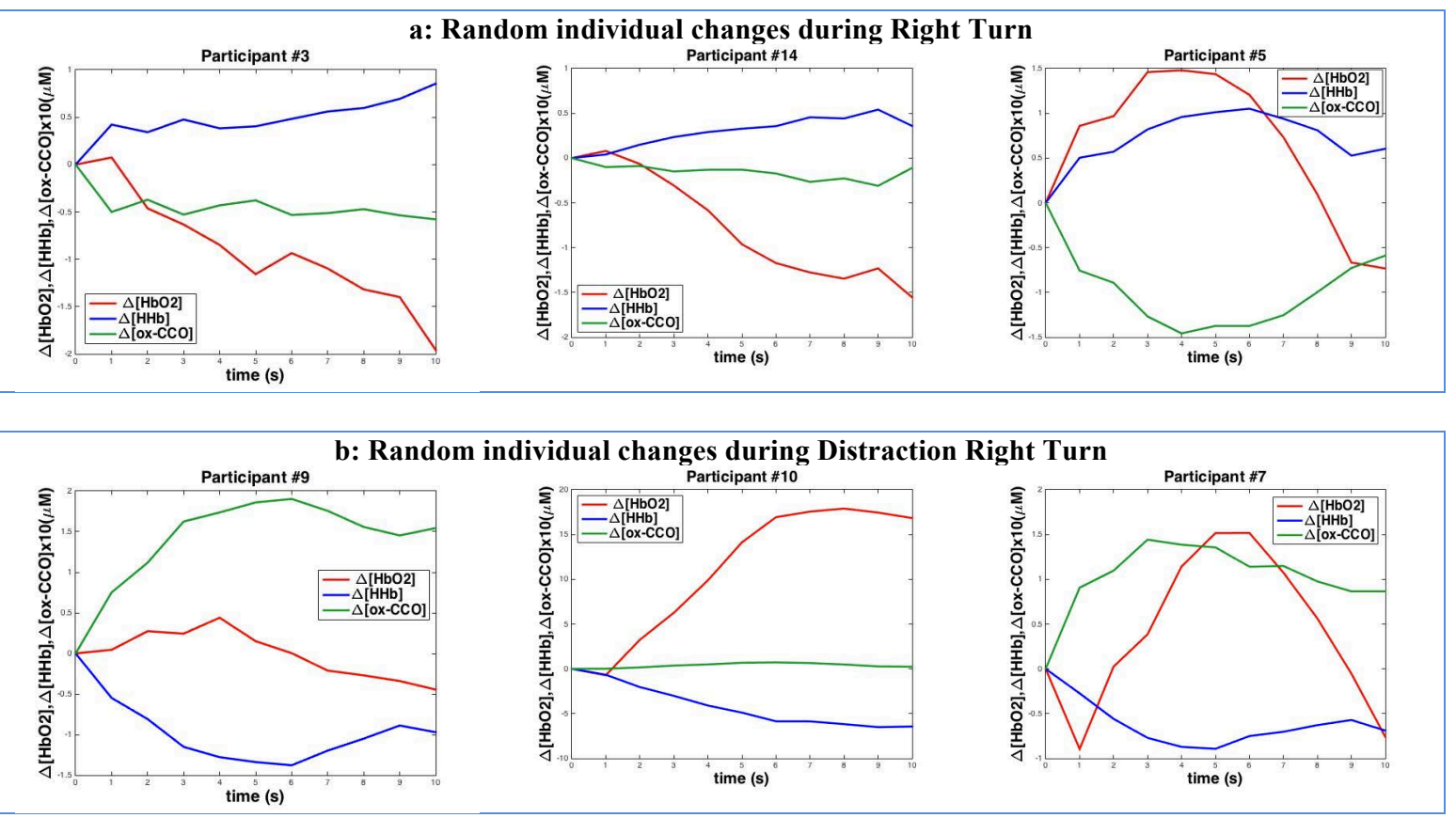

Figure 2-2; Individual Event-related changes during a) right turn b) distracted right turn
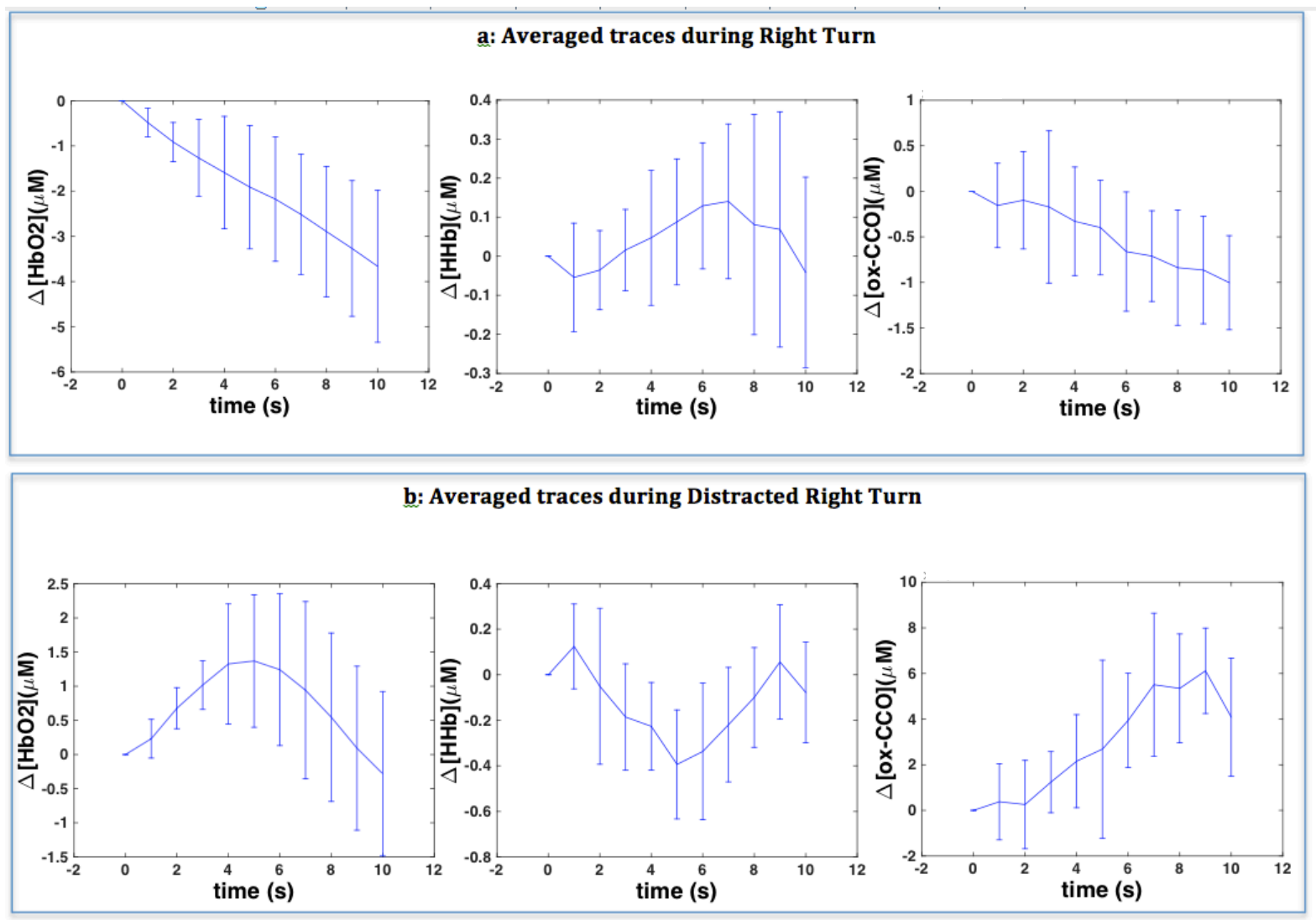

Figure 2-3; Average traces representing concentration changes during a) right turn; b) distracted right turn 


\subsection{3 "Left turn + traffic"}

Figure 2-4-a shows an individual example of the effect of the left turn in traffic on PFC. Random individual changes represent a significant decrease in $\mathrm{HbO} 2$ level which is usually accompanied by a decrease in ox-CCO and an increase in $\mathrm{HHb}$. Figure 2-5-a shows the averaged traces during left turn in traffic (averaged over 16 participants). Left turn in traffic resulted in a significant increaseincrease in $\mathrm{HbO} 2$ in right $\mathrm{PFC}(\mathrm{p}<0.05)$. The rest of the changes in average traces were not statistically significant in either sides.

\subsection{4 "Left turn + traffic + distraction"}

Figure 2-4-b shows an individual example changes during distracted left turn in traffic. Individual trends show a significant increase in $\mathrm{HbO}_{2}$ level accompanied by a decrease in $\mathrm{HHb}$ and an increase in ox-CCO in both sides. Figure 2-5-b shows the averaged traces during distracted left turn in traffic. According to the average traces distracted left turn in traffic resulted in a significant increase in $\mathrm{HbO}_{2}$ along with a decrease in $\mathrm{HHB}$ and an increase in ox-CCO in both right and left PFC $(\mathrm{p}<0.05)$. 

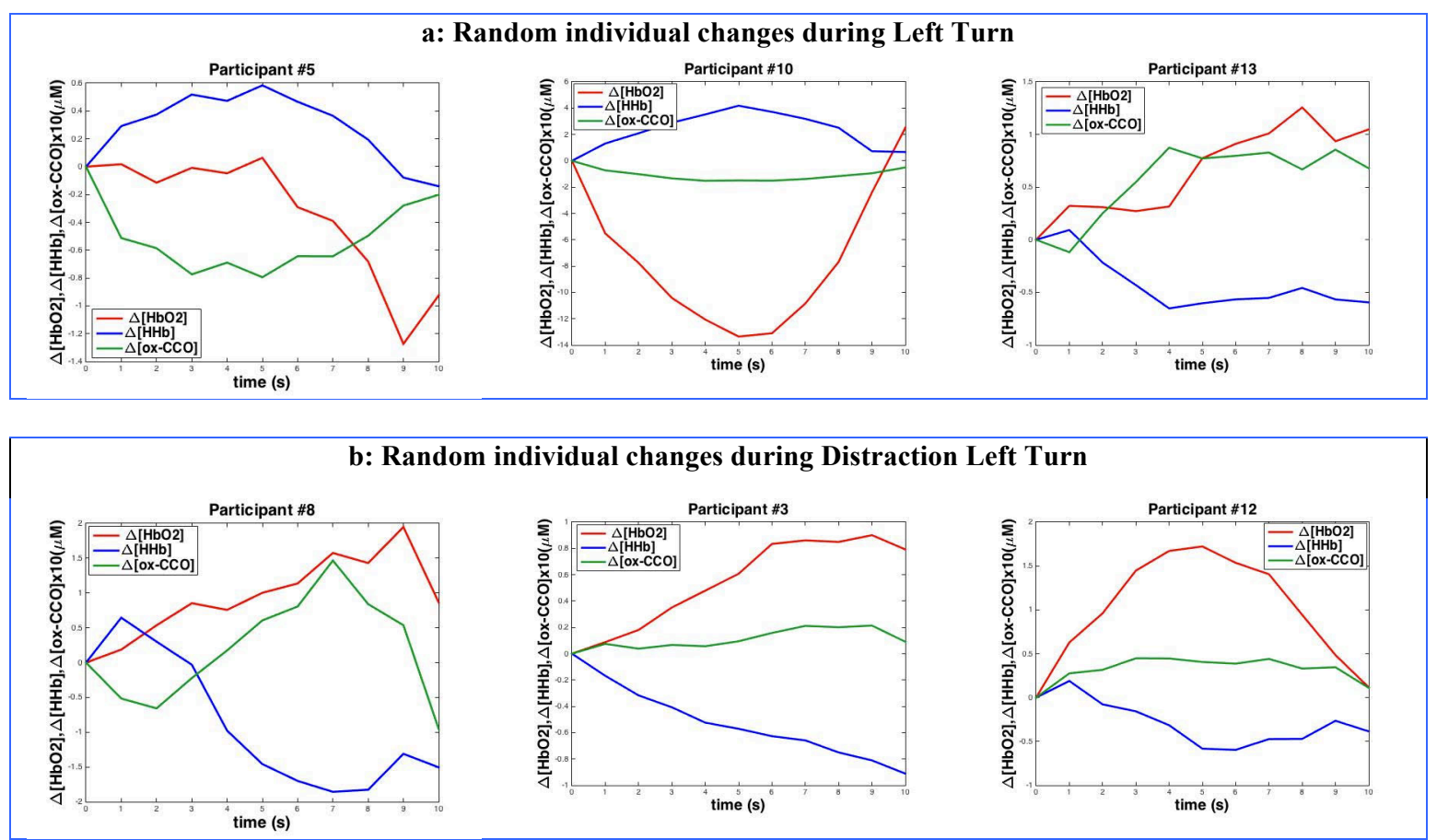

Figure 2-4; Individual event related concentration changes during a) left turn in traffic; b) distracted left turn in traffic
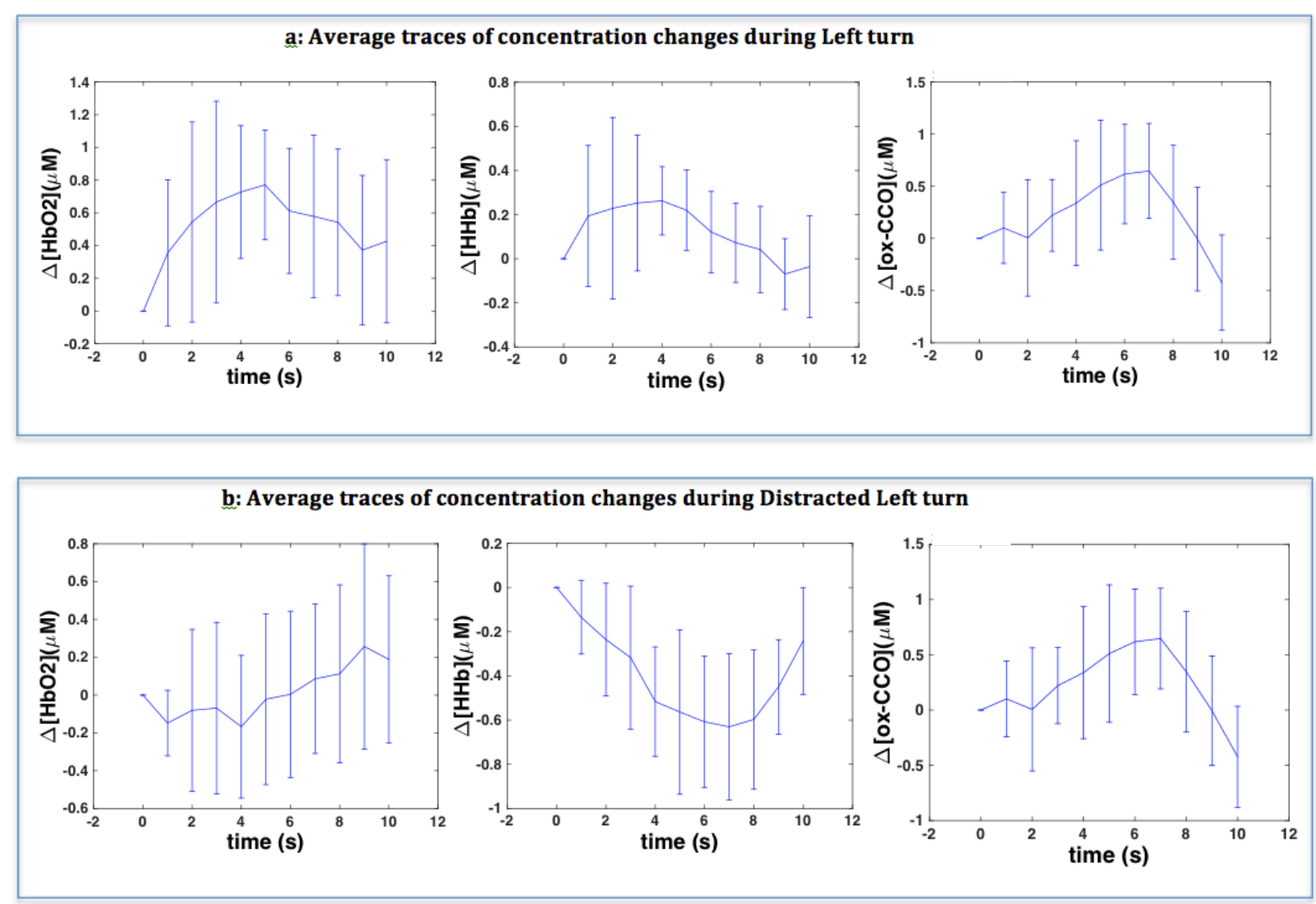

Figure 2-5; Average traces of concentration changes During a) left turn in traffic; b) distracted turn in traffic 


\subsection{5 "Straight + distraction"}

As Figure 2-6-b shows average changes due to distraction during straight driving. It shows that this event resulted in an increase in $\mathrm{HbO}_{2}$ level accompanied with by decreasing $\mathrm{HHb}$ in both right and left PFC $(\mathrm{p}<0.05)$, however changes in ox-CCO were not statistically significant in either side. Some random individual results during distracted straight driving are shown in figure 2-6-a. Similar trends as the average traces are observed in individual changes.
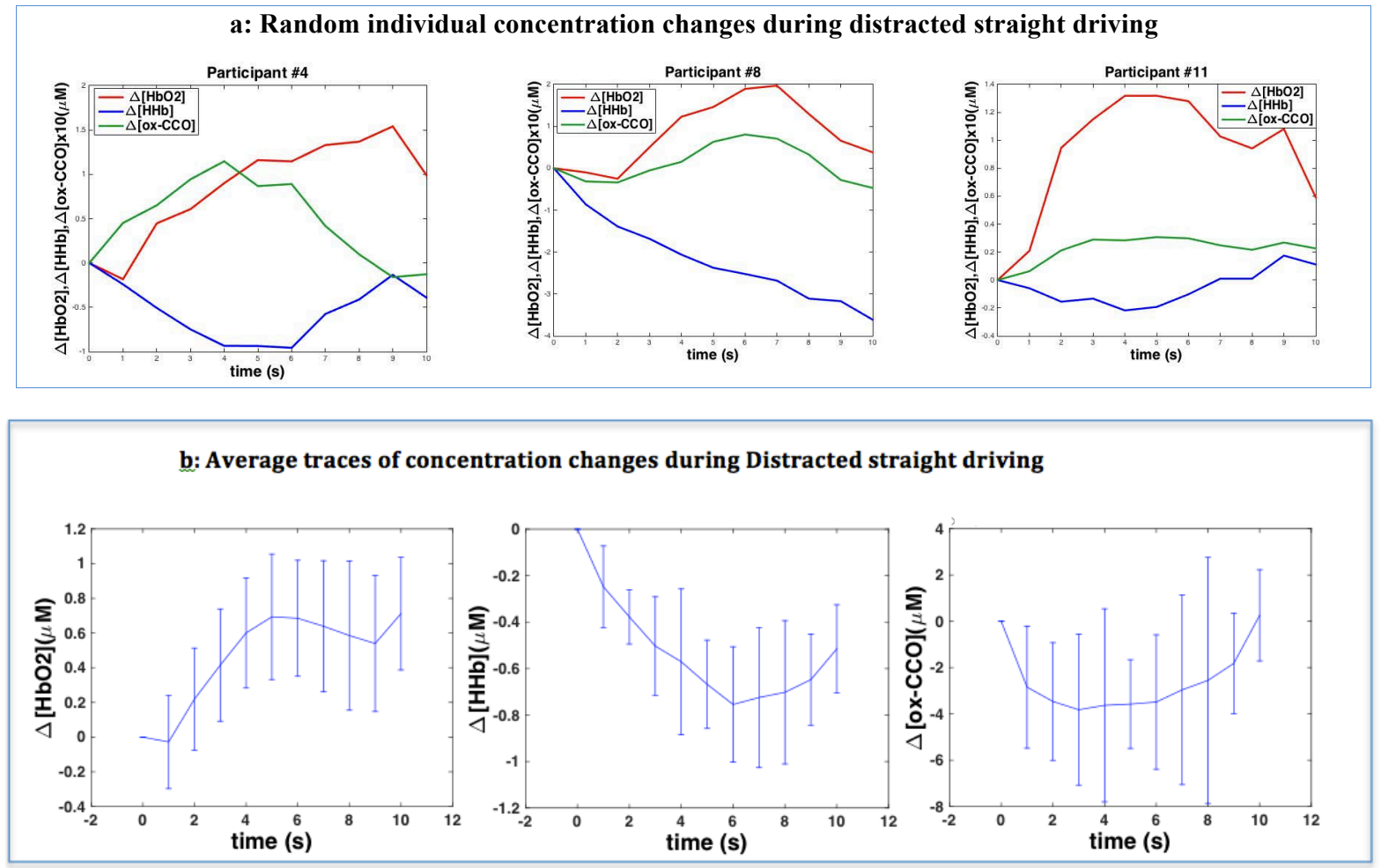

Figure 2-6; a) Individual and b) average changes during distracted straight driving

\subsubsection{Comparing different distracted conditions}

In order to investigate distraction during which driving task has the highest effect in altering brain activation pattern, the changes during "Straight + distraction", "Left turn + 
traffic + distraction" and "Right turn + distraction" are shown in figure 2-7. In right PFC distracted left turn in traffic results in larger changes in $\mathrm{HHb}$ and ox-CCO compared to other distracted conditions. Changes in ox-CCO are more consistent in right PFC.
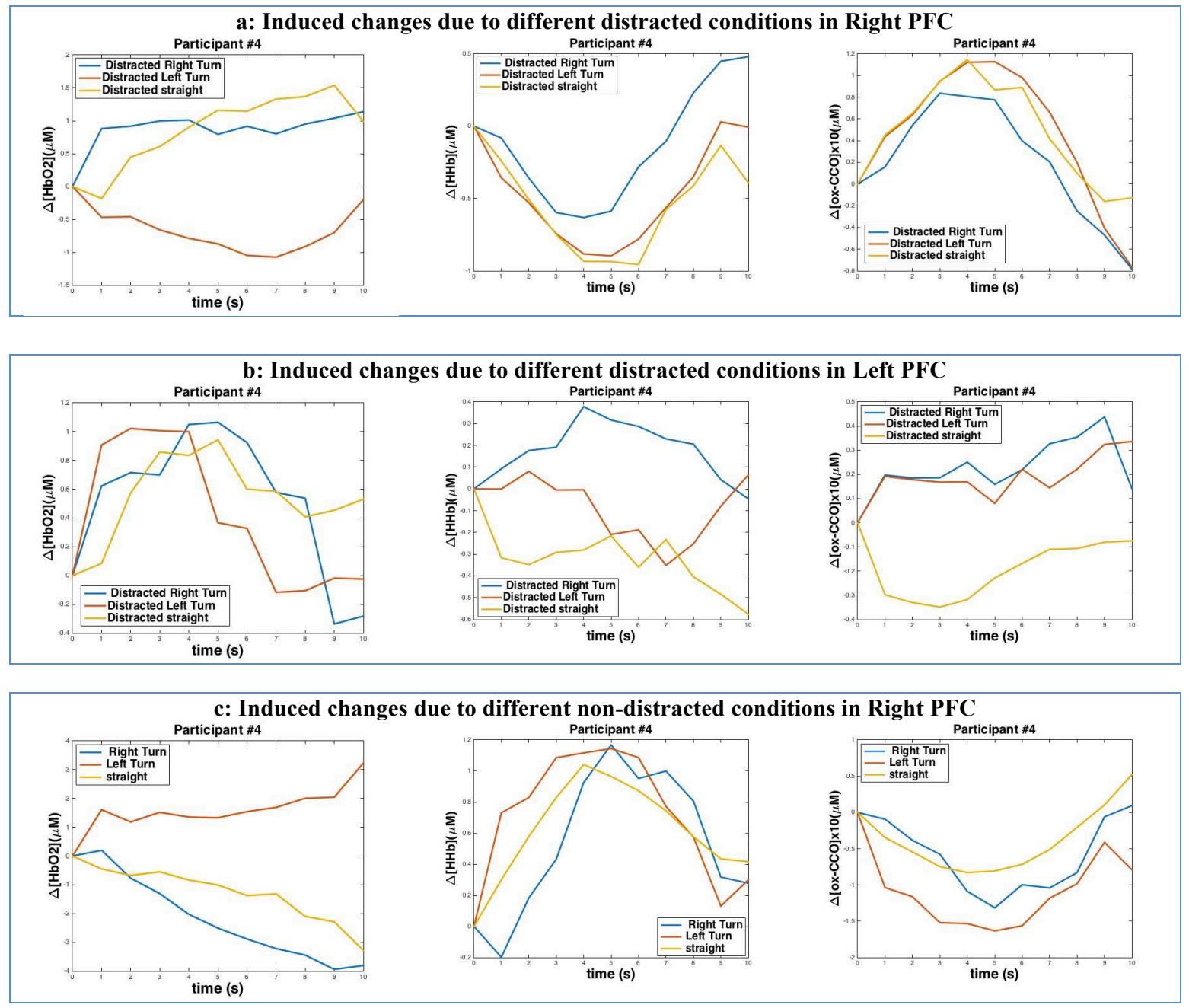

Figure 2-7; Comparing the effect of distraction during different driving tasks in a) Right PFC and b) left PFC of a random subject c) induced changes during different non-distracted driving tasks on the same subject

\subsection{7 "Accident Avoidance"}

In left PFC the average $\mathrm{HbO}_{2}$ concentration decreased but not statistically significant; oxCCO decreased significantly $(\mathrm{p}<0.05)$ and $\mathrm{HHb}$ increased $(\mathrm{p}<0.05)$ and $\mathrm{HHb}$ increased in the left PFC. 
In the right $\mathrm{PFC}_{\mathrm{HbO}}$ concentration decreased significantly $(\mathrm{p}<0.05)$ though the changes in $\mathrm{HHb}$ and ox-CCO were not statistically significant $(\mathrm{p}=0.1)$.

\subsubsection{Results in summary}

All results are summarized in Table 2-1 Right and left PFC were both significantly activated $(\mathrm{p}<0.05)$ during distracted right turn and distracted left turn in traffic similar to our fMRI findings. All activations induced by distractions were identified by increase in $\mathrm{HbO}_{2}$ and were mostly accompanied by decreasing $\mathrm{HHb}$ and increasing ox-CCO. Between all the events "left turn in traffic" resulted in least consistent changes between participants.

Table 2-1; Average event-related changes in concentrations of $\mathrm{HbO2}, \mathrm{HHb}$ and ox-CCO and calculated p-value from paired-sample t-tests (performed between the initial moment of each event and the maximum induced changes); arrows show the direction of the average changes that were statistically significant and the changes that were not statistically significant are shown by .

\begin{tabular}{|c|c|c|c|c|c|c|}
\hline \multirow{2}{*}{ Event } & \multicolumn{2}{|c|}{ HbO2 } & \multicolumn{2}{|c|}{$\mathbf{H H b}$} & \multicolumn{2}{|c|}{ ox-CCO } \\
\hline & Left PFC & Right PFC & Left PFC & Right PFC & Left PFC & Right PFC \\
\hline & $y$ & $y$ & 2 & 2 & $\approx$ & $y$ \\
\hline \multicolumn{7}{|l|}{ Right turn } \\
\hline & $\mathrm{p}$-value $=0.02$ & $\mathrm{p}$-value $=0.04$ & $\mathrm{p}$-value $=0.05$ & $\mathrm{p}$-value $=0.03$ & $\mathrm{p}$-value $=0.3$ & $\mathrm{p}$-value $=0.04$ \\
\hline \multirow{2}{*}{$\begin{array}{c}\text { Right turn + } \\
\text { distraction }\end{array}$} & 2 & 7 & $\approx$ & $y$ & 7 & $\approx$ \\
\hline & $\begin{array}{c}\text { p-value }= \\
0.008\end{array}$ & $\mathrm{p}$-value $=0.01$ & $\mathrm{p}$-value $=0.1$ & $\mathrm{p}$-value $=0.008$ & $\begin{array}{c}\text { p-value }= \\
0.004\end{array}$ & $\mathrm{p}$-value $=0.3$ \\
\hline \multirow{2}{*}{$\begin{array}{c}\text { Left turn }+ \\
\text { traffic }\end{array}$} & $\approx$ & 7 & $\approx$ & $\approx$ & $\approx$ & $\approx$ \\
\hline & $\mathrm{p}$-value $=0.2$ & $\mathrm{p}$-value $=0.04$ & $\mathrm{p}$-value $=0.1$ & $\mathrm{p}$-value $=0.1$ & $\mathrm{p}$-value $=0.1$ & $\mathrm{p}$-value $=0.4$ \\
\hline \multirow{2}{*}{$\begin{array}{c}\text { Left turn }+ \\
\text { traffic }+ \\
\text { distraction }\end{array}$} & 7 & $\approx$ & & & 7 & 2 \\
\hline & $\mathrm{p}$-value $=0.01$ & $\mathrm{p}$-value $=0.4$ & $\mathrm{p}$-value $=0.05$ & $\mathrm{p}$-value $=0.04$ & $\mathrm{p}$-value $=0.01$ & $\mathrm{p}$-value $=0.04$ \\
\hline \multirow{2}{*}{$\begin{array}{l}\text { Straight + } \\
\text { distraction }\end{array}$} & & 2 & & & $\approx$ & $\approx$ \\
\hline & $\mathrm{p}$-value $=0.01$ & $\mathrm{p}$-value $=0.02$ & p-value $=0.002$ & $\mathrm{p}$-value $=0.04$ & $\mathrm{p}$-value $=0.3$ & $\mathrm{p}$-value $=0.3$ \\
\hline
\end{tabular}


A statistical comparison between event-related changes due to distracted vs. nondistracted conditions along with one-way ANOVA results are shown in table 2-2 and figure 2-8. This analysis was performed to generally assess the effect of distraction during driving. "Non-distracted" group contained: "straight", "right turn" and "left turn in traffic"; distracted group contained: "straight + distraction", "right turn + distraction"”, "left turn in traffic + distraction",.

Average changes in $\Delta\left[\mathrm{HbO}_{2}\right], \Delta[\mathrm{HHb}]$ and $\Delta[\mathrm{ox}-\mathrm{CCO}]$ shown in table 2-2 and figure 2-8, in particular positive $\Delta[\mathrm{HHb}]$ and negative $\Delta[\mathrm{ox}-\mathrm{CCO}]$, indicate that left and right turns without distractions deactivate both the left and right PFC. Oppositely, distractions during driving tasks caused activation of both right and left PFC in terms of all three chomophores. Furthermore, ANOVA p-values demonstrated that distracted and nondistracted conditions resulted in statistically significant opposite changes in $\Delta \mathrm{HbO}_{2}$ and $\Delta \mathrm{HHb}(\mathrm{p}<0.05)$ in both right and left PFCs. Furthermore, the directions of $\triangle \mathrm{CCO}$ in right PFC were opposite between distracted and non-distracted conditions $(\mathrm{p}<0.05)$. However, for the left PFC the reference in $\triangle \mathrm{CCO}$ between distracted and non-distracted groups was not statistically significant $(\mathrm{p}=0.7)$.

Based on the results show that distractions during the left turn in traffic, a more complex driving task compared to right turn, resulted in greater activation in the right PFC. 
Table 2-2; Average concentration changes and one-way ANOVA results of all distracted conditions vs. average changes of all non-distracted conditions; positive means reflect an overall increase and negative means represent an overall decrease. The mean changes in right $\mathrm{PFC}$ are also shown in the bar graph. (*in the bar graph ox-CCO changes has been multiplied by a factor of 107)

\begin{tabular}{|c|c|c|c|c|c|c|}
\hline & \multicolumn{2}{|c|}{$\mathrm{HbO}_{2}$} & \multicolumn{2}{|c|}{$\mathrm{HHb}$} & \multicolumn{2}{|c|}{$\mathrm{ox}-\mathrm{CCO}$} \\
\hline \multirow{5}{*}{ Right PFC } & \multicolumn{6}{|c|}{ Concentration changes Mean (micro Molar) } \\
\hline & $\begin{array}{c}\text { Non- } \\
\text { Distracted }\end{array}$ & Distracted & $\begin{array}{c}\text { Non- } \\
\text { Distracted }\end{array}$ & Distracted & $\begin{array}{c}\text { Non- } \\
\text { Distracted }\end{array}$ & Distracted \\
\hline & -0.0644 & 0.6938 & 0.2557 & -0.3985 & $-8.09 \mathrm{E}-08$ & $3.91 \mathrm{E}-08$ \\
\hline & \multicolumn{6}{|c|}{ p-value for distracted ys non-distracted } \\
\hline & \multicolumn{2}{|c|}{0.039} & \multicolumn{2}{|c|}{0.0088} & \multicolumn{2}{|c|}{0.01} \\
\hline \multirow{5}{*}{ Left PFC } & \multicolumn{6}{|c|}{ Concentration changes Mean (micro Molar) } \\
\hline & $\begin{array}{c}\text { Non- } \\
\text { Distracted }\end{array}$ & Distracted & $\begin{array}{c}\text { Non- } \\
\text { Distracted }\end{array}$ & Distracted & $\begin{array}{c}\text { Non- } \\
\text { Distracted }\end{array}$ & Distracted \\
\hline & -2.1402 & 0.2301 & 0.1772 & -0.2899 & $-1.58 \mathrm{E}-08$ & $-3.06 \mathrm{E}-09$ \\
\hline & \multicolumn{6}{|c|}{ p-value for distracted ys non-distracted } \\
\hline & \multicolumn{2}{|c|}{0.04} & \multicolumn{2}{|c|}{0.045} & \multicolumn{2}{|c|}{0.7} \\
\hline
\end{tabular}

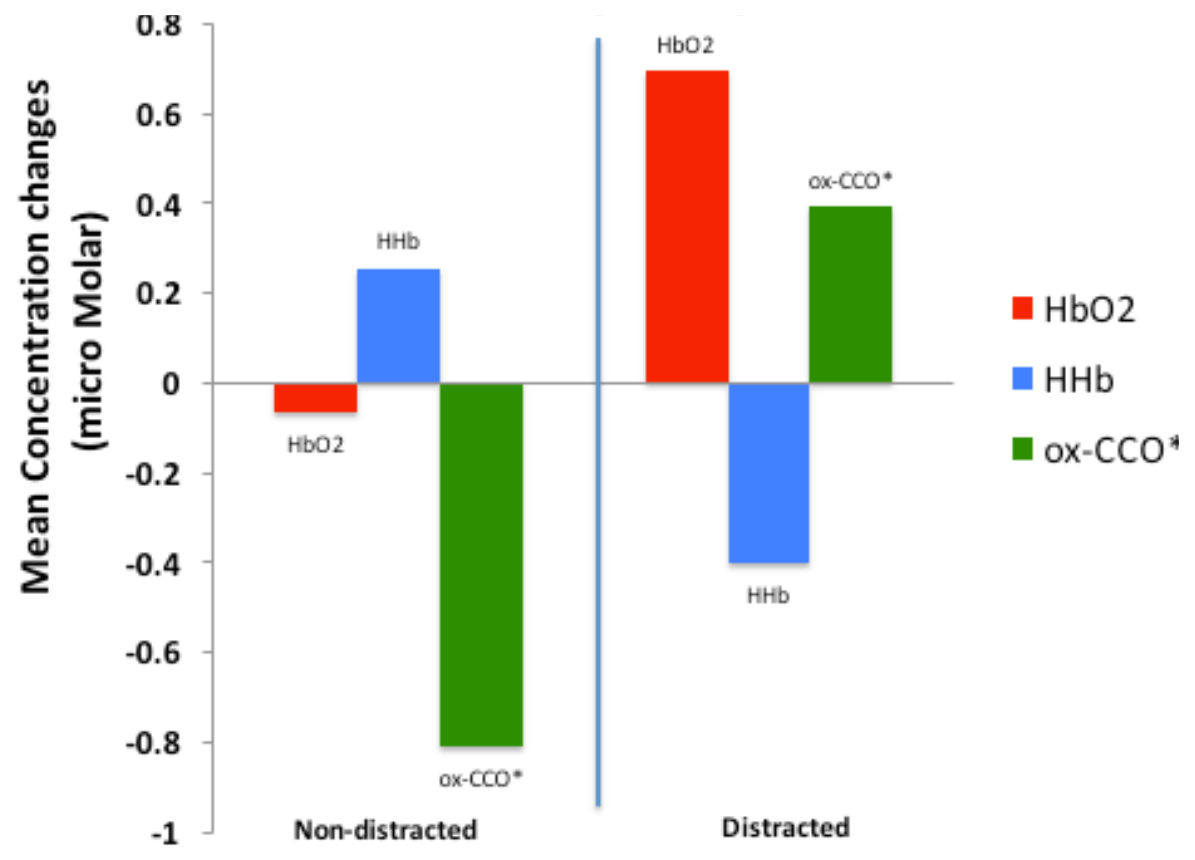

Figure 2-8; Average induced changes during non-distracted conditions versus distracted conditions; mean changes have been calculated with ANOVA between all distracted conditions (including Left turn + distraction, Straight + distraction and Right turn + distraction) and non-distracted conditions (including Left turn, Straight and Right turn); * ox-CCO values have been multiplied by 10 


\subsection{Discussion}

This study was designed to measure event-related activations changes in right and left prefrontal cortices during different driving conditions. Five different driving events were investigated: "straight + distraction", "right turn", "right turn + distraction", "left turn in traffic", and "accident avoidance left turn in traffic + distraction" and "straight + distraction".

An increase in $\mathrm{HbO}_{2}$ concomitant with a decrease in $\mathrm{HHb}$ during a cognitive task reflects oxygen supply enhancement due to increased blood flow in response to the brain activation that was accompanied by an increase in $\mathrm{Ox}-\mathrm{CCO}$ representing tissue oxygen consumption increase (Kolyva,2012).

In all distracted conditions PFC activation (increased oxygen delivery) was observed along with an increase in ox-CCO. PFC was activated due to distractions during driving and resulted in an increase in both oxygen consumption and oxygenated hemoglobin supply. Although in this study we did not measure the occipital lobe that is responsible for the visual attention, the previous fMRI study has shown that distractions activate the PFC accompanied by loss of enhanced activation in the occipital lobe required for higher visual attention in complex driving tasks (Schweizer TA, 2013). Indeed distraction sacrifices occipital brain that is responsible for visual attention and increases the risk of accident (Hauke R Heekeren, 1999).

Making "right turn" significantly deactivated both right and left PFC; ox-CCO decreased and $\mathrm{HHb}$ increased following the deactivation $(\mathrm{p}<0.05)$. The changes during "left turn + traffic" were not statistically significant, however, the average shows that left PFC was deactivated while right PFC was activated. 
Previously published fMRI data collected during similar driving tasks (Schweizer TA, 2013) has shown activation in the right and left PFC during all distracted driving conditions, which agrees with our current findings using NIRS. No PFC activation was observed in fMRI results during right or left turn that also agrees with our findings. Based on increased $\mathrm{HbO}_{2}$ the right PFC activated significantly when participants tried to avoid an accident while the left PFC deactivated according to a significant increase in $\mathrm{HHb}$ in the left $\mathrm{PFC}$; it suggests that in conditions requiring very fast and unexpected planning and reflexive tasks such as higher visual attention and motor function response (pushing foot pedals or turning the steering wheel) right PFC is highly activated [14].

Unfortunately, the data variance in this study was rather high so that only group results were statistically significant. The reasons for that were the event-related nature of relatively subtle cerebral changes, the biasing influence of extra-cerebral hemodynamics on NIRS data, and small number of fNIRS channels. Increasing of the number of channels to measure other regions of the brain simultaneously (specially posterior lobe which is responsible for visual attention) with research on advanced fNIRS signal processing (to enhance SNR and better isolate different chromophores) in future may result in feasibility of detecting event-related responses in individual subjects.

Overall, the ANOVA statistical analysis of the behavior of all three chromophores shows that distractions activate both left and right PFC, which is in strong agreement with fMRI results (Schweizer TA, 2013).

\subsection{Conclusions}

In this study we used hyperspectral fNIRS to investigate the event-related activity in right and left prefrontal cortices during various driving conditions. Based on our previous 
fMRI study we expected most significant activations during distractions. All cases of activation exhibited the typical activation pattern of increased $\mathrm{HbO} 2$ with simultaneously decreased $\mathrm{HHb}$ (with the exception of left turn in traffic in the right PFC), which provides an additional confirmation of the activation. Furthermore, expected responses of $\Delta[\mathrm{oxCCO}]$ to functional activations were observed in most cases of distractions. Namely, $\Delta[\mathrm{oxCCO}]$ increased concurrently with the increase in $\mathrm{HbO} 2$ except for "straight + distraction" condition in the left PFC. Such a consistent behavior of $\Delta[\mathrm{oxCCO}]$ indicates that hyperspectral fNIRS can directly assess not only the vascular activity but also the event-related changes in cerebral oxygen metabolism.

Our results dovetail nicely with our previous fMRI findings using similar driving scenarios (Schweizer TA, 2013). However, in order to better understand the relationship between activation of different cortical regions, in the future we need to perform NIRS measurements on different positions simultaneously. Overall our findings prove that fNIRS is a useful technique to measure brain activity during actual driving; fNIRS can also be developed to identify which cognitive or non-cognitive tasks during driving (such as eating, drinking, speaking and texting) may result in distracted driving conditions (activation of PFC that may sacrifice visual attention). 


\section{Chapter 3 \\ Journal manuscript 2}

\section{Simultaneous measurement of cerebral and muscle tissue parameters during cardiac arrest and cardiopulmonary resuscitation}

Reyhaneh Nosrati ${ }^{a^{*}}$, Steve Lin, Xudong. Hu ${ }^{\mathrm{b}}$, Siwook Kim ${ }^{\mathrm{a}}$, Paul Dorian ${ }^{\mathrm{b}, \mathrm{c}, \mathrm{d}}$ and Vladislav Toronov ${ }^{\mathrm{a}}$

${ }^{a}$ Departments of Physics, Ryerson University, 350 Victoria St, Toronto, Ontario, Canada, M5B 2K3 reyhaneh.nosrati@ryerson.ca; toronov@ryerson.ca

${ }^{\mathrm{b}}$ Keenan Research Centre of the Li Ka Shing Knowledge Institute, St. Michael's Hospital,30 Bond Street,Toronto, Ontario, Canada, M5B 1W8;

${ }^{\mathrm{c}}$ Department of Medicine and Cardiology, University of Toronto, St. Michael's Hospital,30 Bond Street, Toronto, Ontario, Canada, M5B 1W8;

${ }^{\mathrm{d}}$ School of Engineering Technology and Applied Science, Centennial College, 941 Progress Ave, Toronto, Ontario, Canada M1G 3T8

\subsection{Abstract}

In this series of animal experiments on resuscitation after cardiac arrest we had a unique opportunity to measure hyperspectral near-infrared spectroscopy (hNIRS) parameters directly on the brain dura, or on the brain through the intact pig skull, and simultaneously the muscle hNIRS parameters. Simultaneously the arterial blood pressure and carotid and femoral blood flow were recorded in real time using invasive sensors. We used a novel hyperspectral signal-processing algorithm to extract time-dependent concentrations of water, hemoglobin, and redox state of cytochrome c oxidase during cardiac arrest and resuscitation. In addition in order to assess the validity of the non-invasive brain 
measurements the obtained results from the open brain was compared to the results acquired through the skull. The comparison of hNIRS data acquired on brain surface and through the adult pig skull shows that in both cases the hemoglobin and the redox state cytochrome $\mathrm{c}$ oxidase changed in similar ways in similar situations and in agreement with blood pressure and flow changes. The comparison of simultaneously measured brain and muscle changes showed expected differences. Overall the results show feasibility of transcranial hNIRS measurements cerebral parameters including the redox state of cytochrome oxidase in human cardiac arrest patients.

\section{Keywords:}

Cardiac arrest, Ventricular Fibrillation (VF), Oxy-hemoglobin (HbO2), Deoxyhemoglobin ( $\mathrm{HHb})$, Cytochrome c oxidase (CCO), Hyperspectral, Near Infrared Spectroscopy (NIRS), Cardiopulmonary resuscitation (CPR)

\subsection{Introduction}

Cardiac arrest is a sudden stop of effective blood circulation. Ventricular fibrillation (VF) is the worst form of cardiac arrest that is due to ineffective contractions of the muscles of the lower chambers of the heart (ventricles) that results in very rapid, uncoordinated and ineffective series of contractions. In such condition ventricles are quivering (fibrillating) rather that effective contraction thus cannot pump the blood to the peripheral tissue resulting in ischemic condition. The brain is the most sensitive organ to under-perfusion during VF. Cardio Pulmonary Resuscitation (CPR) and defibrillation (electrical shock) are the first treatments that have to be done to recover the patients from VF. Brain damage or death during cardiac arrest are the main concerns that are mainly due to two factors: decreasing oxygen delivery (oxygen supply) and reduction of cellular oxygen 
metabolism (oxygen demand) [47]. Monitoring brain oxygenation and metabolism changes during cardiac arrest and assessment of the effects of CPR, defibrillation and medication on recovery of the brain is very useful and can identify the most effective resuscitation protocol that results in highest survival rate and minimal brain hypoxia.

Hyperspectral Near Infrared Spectroscopy (hNIRS) is a unique technique that can monitor living tissue metabolism and oxygenation level with high temporal resolution.

Tissue oxygen supply and demand could be monitored by real time measurement of intravascular oxygenated hemoglobin $(\Delta[\mathrm{HbO} 2])$ that reflects changes in oxygen delivery and intracellular oxidized-reduced cytochrome $\mathrm{c}$ oxidase $(\Delta[\mathrm{Cyt}-\mathrm{ox}])$ representing mitochondrial oxygen metabolism $[48,49,50]$. Previous studies have shown that ox-CCO response provides higher brain specificity than oxyhemoglobin [51]. Increasing cerebral oxygen delivery is not always accompanied by an increase in ox-CCO and vice versa [52]. As ox-CCO concentration changes directly reflects intracellular oxygen synthesis rather than intravascular oxygen supply, measuring ox-CCO gives us more useful information about cerebral metabolic status and oxygen utilization [52].

Cyt-ox is the terminal electron acceptor in the electron transport chain located in the inner membrane of the mitochondria.. Mitochondrial cytochrome $\mathrm{c}$ has four redox active metal centers, two haem and two coppers $(\mathrm{CuA}$ and $\mathrm{CuB})$. $\mathrm{CuA}$ center has a strong absorbance in NIR spectrum [53]. In the last step of the electron transport chain Cyt-ox accepts an electron from cytochrome $\mathrm{c}$ and transfers it to an oxygen molecule and along with hydrogen ion; oxygen is converted into water molecule. During this process flow of electrons in electron transport chain results in accumulation of $\mathrm{H}+$ ions in the intramembranous space of the mitochondria (the space between inner and outer 
membrane) and eventually when they are diffused back into the mitochondrial matrix, their energy is utilized by ATP synthase to produce ATP molecules. Electron transport chain is responsible for $95 \%$ of the oxygen metabolism and ATP synthesis $[\mathbf{5 4 , 5 5}]$

The total concentration of the $\mathrm{CCO}$ is almost constant over a short period of time therefore its changes is not detectable by NIRS however CuA center accepts and denotes an electron very fast and changes between reduced and oxidized state rapidly [56,57]; the redox changes (oxidized minus reduced) of $\mathrm{CuA}(82 \%$ oxidized) has a distinct absorption band centered around $830 \mathrm{~nm}[\mathbf{5 8 , 5 9 , 6 0}]$.

Extinction coefficient of the oxidized state of $\mathrm{CCO}$ is relatively larger than those of in $\mathrm{HHb}$ and $\mathrm{HbO} 2$ however the fact that the concentration of $\mathrm{CCO}$ is almost $10 \%$ of oxygenated and deoxygenated hemoglobin makes detection of it very challenging.

In the present study we have used a novel signal-processing algorithm to extract concentration changes of $\mathrm{HbO} 2, \mathrm{HHb}$ and ox-CCO during cardiac arrest while different resuscitation protocols were performed. We simultaneously monitored brain and leg muscle by hNIRS and compared the chromophores' concentration changes in the muscle and the brain.

Hyperspectral NIRS offers significant advantages over the multispectral NIRS. The technique appears to be more sensitive to cerebral hemodynamic changes (by monitoring oxygenated and deoxygenated hemoglobin) than the multispectral NIRS and even capable to directly assess neuronal oxygen metabolism by measuring redox state of the cytochrome c oxidase. 


\subsection{Materials and Methods}

NIR signal was collected simultaneously from the brain and the biceps femoris muscle of the leg. In addition brain signal was measured invasively while optodes were placed on the dura mater (after removing the skin and skull at the region of measurement) and also non-invasively by placing the optodes over the skull. We used an in-house continuous wave broadband spectroscopy system. Brain signal acquisition was performed using a broadband spectrometer with spectral range of 650-1100 nm (QE65000 Ocean Optics, Dunedin, FL) consisting of a holographic grating and a cooled charge-coupled device (CCD); muscle data was collected by a lower resolution spectrometer (USB4000 Ocean Optics, Dunedin, FL) with 3648-element CCD array detector. A stabilized fan-cooled tungsten halogen lamp (Avantes Inc., Broomfield, $\mathrm{CO}$ ) was used as a broadband source. 1-m long optical fiber bundles with $5 \mathrm{~mm}$ core diameter were used to transmit the source light to the tissue and also detect the transmitted light through the tissue and transfer it to the spectrometer.

Source detector distance for the brain was $30 \mathrm{~mm}$ and for the muscle was $20 \mathrm{~mm}$. To keep the optodes in place during CPR and defibrillation, punched $7 \mathrm{~mm}$ thick rubber pads with two holes (with 20 and $30 \mathrm{~mm}$ spacing for the muscle and brain optodes respectively) were used. In addition the cloth tape around the fibers was sutured to the surrounding skin layer. The sampling frequency was $4 \mathrm{~Hz}$ (integration time of $250 \mathrm{~ms}$ ) for all detectors.

The changes in chromophores' concentration were extracted from the acquired data using a novel multi layer stepwise algorithm. Our data processing algorithm was basically based on the fitting of the absorbance changes by modeling absorbance using the 
analytical solution to the diffusion equation for the semi-infinite medium with an extrapolated boundary condition. In our algorithm after determining the baseline values for each chromophore, reduced scattering coefficient is calculated for every wavelength by the power law as described in more detail in [61]. The concentration changes of different chromophores were calculated by non-linear fitting of the flux changes to the absorbance using the Matlab function 'Isqcurvefit', where the parameters of the fit were $\Delta[\mathrm{HbO} 2], \Delta[\mathrm{HHb}], \Delta[\mathrm{ox}-\mathrm{CCO}]$ and $\Delta[\mathrm{H} 2 \mathrm{O}]$. Data fitting was performed is 3 different steps with different spectral bands. In the first step data fitting was performed over the spectral band of $700 \mathrm{~nm}$ to $800 \mathrm{~nm}$ and $\Delta[\mathrm{HbO} 2], \Delta[\mathrm{HHb}]$ were estimated. The second wave band was $900 \mathrm{~nm}$ to $990 \mathrm{~nm}$ to determine $\Delta[\mathrm{H} 2 \mathrm{O}]$ concentration. Finally in the last step spectral range of $700 \mathrm{~nm}$ to $900 \mathrm{~nm}$ was used to estimate $\Delta$ [ox-CCO]. This stepwise approach significantly improved the results of the study.

All protocols were approved by the Animal Care Committee of St. Michael's Hospital (Toronto, ON, Canada) and conformed to the guide for the care and use of laboratory animals, US National Institutes of Health (NIH Publication $\mathrm{N}^{\circ}$. 85-23, revised 1996). The ARRIVE guidelines were used as the guidelines for carrying out experiments and presenting the information.

Pigs (Yorkshire, either sex, 25-35 kg) were fasted overnight and sedated with ketamine $(20 \mathrm{mg} / \mathrm{kg}$ ), xylazine $(2 \mathrm{mg} / \mathrm{kg}$ ) and atropine (1ml/25 kg) (all IM) and anesthesia was induced with 5\% isoflurane. Once anesthetized, pigs were endotracheally intubated and maintained by continuous administration of isoflurane ( $1 \%-3 \%$ mixed with $100 \% \mathrm{O} 2)$.

Pigs were connected to a ventilator (Ohio ventilator R.A.E. Technologies, Inc. Ontario) at a rate: 21 breaths $/ \mathrm{min}$, tidal volume: $10-15 \mathrm{ml} / \mathrm{kg}$, minute ventilation: $5 \mathrm{~L} / \mathrm{min}$, and $\mathrm{I}: \mathrm{E}$ 
ratio of 1:2. End-tidal $\mathrm{CO} 2$ was continuously measured using a CO2MO Plus monitoring system (Philips Healthcare, Andover, MA) and was kept at $35-45 \mathrm{mmHg}$. Depth of anesthesia was checked periodically (every 15 minutes) by monitoring heart rate, end tidal $\mathrm{CO} 2$, and evaluating muscle tone, jaw reflex and movements. No procedures were performed until pigs were at a surgical plane of anesthesia. Ringers lactate infusion at a rate of $2-4 \mathrm{ml} / \mathrm{kg} / \mathrm{hr}$ was administered as maintenance fluid. A baseline arterial blood sample $(1 \mathrm{~mL})$ was taken for blood gas analysis.

Other parameters that were being monitored during the experiment contained: heart rate (HR), electroencephalograph, carotid and femoral artery blood flow and blood pressure and arterial oxygen saturation.

The left femoral artery and vein were catheterized for placement of two solid-state high fidelity, micro manometer-tipped catheters (Mikro-Tip ${ }^{\mathrm{TM}}$ Transducer, Millar Instruments Inc., Houston, TX) for measuring arterial blood pressure; One was placed in the abdominal aorta and the other in femoral artery.

The right carotid artery and right femoral artery were exposed for placement of perivascular flow probes to monitor blood flow and blood pressure (TS420 Perivascular Flowmeter Module, Transonic T403, Transonic Systems Inc., Ithaca, NY). Three limb leads were placed for ECG recording. An electrode catheter (EP Technologies Inc., Sunnyvale, CA) was positioned at the apex of the right ventricle via the right femoral vein to allow for intracardiac ECG recording and the induction of ventricular fibrillation (VF). The electrode was guided to the right ventricle through inferior vena cava and right atrium; it induced the cardiac arrest by applying 10 Volts and disturbing the cardiac normal electrical activity. 
Two self-adhesive defibrillation pads were placed on the left and right chest wall laterally for ventricular defibrillation and connected to an external biphasic defibrillator (PhysioControlLifepak 12, Medtronic Inc., Redmond, WA).

Cardiac arrest was defined by identification of VF on ECG and absent pulsatile aortic systolic pressure. The pig remained in cardiac arrest for 6 minutes followed by 8 to 14 minutes of CPR. Chest compressions were performed by a LUCAS device (98-102 compressions/min, Jolife AB, Lund, Sweden). Ventilation consisted of 6 breaths/min delivered with an Ambu bag (Ambu Inc., Glen Burnie, MD). After the CPR, a defibrillation shock was applied using 150J then CPR was resumed for another 10 to 30 seconds before defibrillation was attempted again with a $200 \mathrm{~J}$ shock, this repeated for a 300J shock. If VF persisted, 30 second intervals of CPR were performed between 360J shocks $(\mathrm{x} 4)$. The experiment was terminated after 4 to 6 unsuccessful shock. Arterial blood samples for blood gas analysis were drawn after 2 minutes of CPR, immediately following the final shock, and 5, 15, 30 and 60 minutes after a successful shock $(1 \mathrm{~mL}$ each). Survived pigs were sacrificed by re-induction of VF after the recovery period had elapsed, or after 5 minutes of a systole or pulseless electrical activity. Epinephrine boluses were infused along with CPR and defibrillation.

Tissue oxygenation and metabolism level were monitored simultaneously on the muscle, exposed brain (on the dura mater) and over the skull.

Different resuscitation protocols by varying by untreated VF duration, CPR duration, number of defibrillations and number of epinephrine infusions were assessed. Brain auto regulation system was investigated by comparing the muscle and the brain tissue oxygenation level. Tissue oxygen saturation was calculated as 
$[\mathrm{HbO} 2 /(\mathrm{HbO} 2+\mathrm{HHb})]^{*} 100$ and the correlations between the arterial blood pressure (measured directly during the experiment) blood oxygenation and ox-CCO were investigated.

In addition in order to assess the validity of the non-invasive brain measurements the obtained results from the open brain was compared to the results acquired through the skull.

\subsection{Results}

\subsubsection{Comparison between invasive vs. semi-invasive cerebral NIRS}

Table 2-3 and 2-4 show the measured changes following VF and CPR in two random animals for both invasive (probing the brain over the dura mater) and semi-invasive (probing the brain over the skull) cerebral NIRS measurements.

Paired-sample t-test (two-tailed, two degree of freedom) was used to investigate the statistically significant difference between dura and skull measurements due to VF and CPR; by conventional criteria, the difference is considered to be not statistically significant $(\mathrm{P}>0.85)$.

Table 3-1; Changes from the baseline to the end of drop/rise after VF induction on NIRS measured signals and mean aortic blood pressure

\begin{tabular}{|c|c|c|c|c|c|c|c|c|c|}
\hline \multirow[t]{2}{*}{ Animal } & \multicolumn{2}{|c|}{$\begin{array}{c}\Delta\left[\mathrm{HbO}_{2}\right] \\
(\mu \mathrm{M})\end{array}$} & \multicolumn{2}{|c|}{$\begin{array}{c}\Delta[\mathbf{H H b}] \\
(\mu \mathrm{M})\end{array}$} & \multicolumn{2}{|c|}{$\begin{array}{c}\Delta[\text { Cyt-ox }] \\
(\mu \mathrm{M})\end{array}$} & \multicolumn{2}{|c|}{$\begin{array}{c}\Delta \mathrm{tSO}_{2} \\
(\%)\end{array}$} & \multirow[t]{2}{*}{$\begin{array}{l}\Delta(\mathrm{mAoP}) \\
(\mathrm{mm} \mathrm{Hg})\end{array}$} \\
\hline & Dura & Skull & Dura & Skull & Dura & Skull & Dura & Skull & \\
\hline 1 & -4.49 & -5 & 7.36 & 9. & -1.7 & -2 & -11.5 & -12.4 & \\
\hline 2 & -11.73 & -12.4 & 12.63 & 8.62 & -3.1 & -3.07 & -16 & -14.1 & -57.36 \\
\hline
\end{tabular}


Table 3-2; The impact of CPR on different cerebral parameters measured by NIRS through the skull (semi-invasively) and dura (invasively) and mean aortic pressure (changes are calculated as the difference right before and maximum changes during CPR)

\begin{tabular}{|c|c|c|c|c|c|c|c|c|c|}
\hline \multirow[t]{2}{*}{ Animal } & \multicolumn{2}{|c|}{$\begin{array}{c}\Delta\left[\mathrm{HbO}_{2}\right] \\
(\mu \mathrm{M})\end{array}$} & \multicolumn{2}{|c|}{$\begin{array}{c}\Delta[\mathbf{H H b}] \\
(\mu \mathrm{M})\end{array}$} & \multicolumn{2}{|c|}{$\begin{array}{c}\Delta[\text { Cyt-ox }] \\
(\mu M)\end{array}$} & \multicolumn{2}{|c|}{$\begin{array}{c}\Delta \mathrm{tSO}_{2} \\
(\%)\end{array}$} & \multirow[t]{2}{*}{$\begin{array}{l}\Delta(\mathrm{mAoP}) \\
(\mathrm{mm} \mathrm{Hg})\end{array}$} \\
\hline & Dura & Skull & Dura & Skull & Dura & Skull & Dura & Skull & \\
\hline 1 & 15.69 & 7.94 & -7.81 & -3.4 & 1.65 & 1.41 & 14.25 & 6.63 & 24.35 \\
\hline 2 & 2.31 & 6.53 & -1.63 & -0.59 & 0.99 & 0.46 & 2.72 & 4.01 & 18.51 \\
\hline
\end{tabular}

Figure 3-1 shows sample NIRS results of simultaneous invasive and semi-invasive cerebral measurements during VF followed by CPR and defibrillation. The general trend of changes (time course of the signals) was identical for all chromophores including $[\mathrm{HbO} 2],[\mathrm{HHb}]$ and redox [Cyt-ox]; the main difference is in the amplitude of changes.

After VF measured [HbO2] and [HHb] through dura is very close to that of measured through skull $(\mathrm{P}>0.8)$. The impact of $\mathrm{CPR}$ on $\Delta[\mathrm{HbO} 2]$ and $\Delta[\mathrm{HHb}]$ was greater when measured invasively than that of measured through skull, however the difference was not statistically significant $(\mathrm{P}>0.85)$

The changes in redox [Cyt-ox] measured through the skull has a greater decline when VF is induced but when CPR begins the recovery of the redox [Cyt-ox] to the normal level are greater when measured through the dura mater. Following the VF the mean $\Delta[$ Cyt-ox] measured through the skull and dura are $-3.01 \mu \mathrm{M} / \mathrm{L}$ and $-2.6 \mu \mathrm{M} / \mathrm{L}$ respectively. 

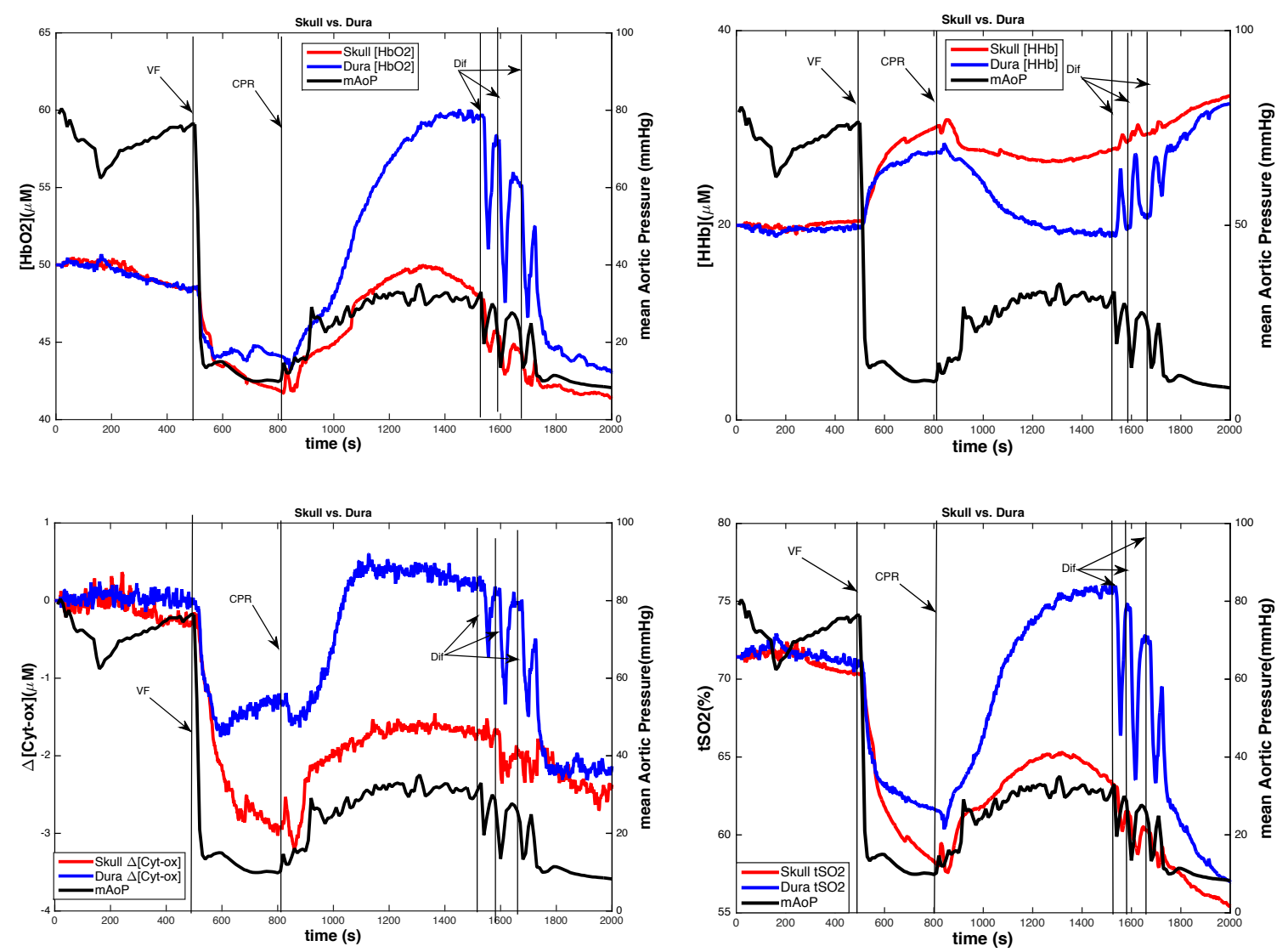

Figure 3-1; [HbO2], [HHb], [Cyt-ox] and tSO2 changes measured by NIRS both invasively (Dura) and semi-invasively (Skull) and mean aortic blood pressure during cardiac arrest and resuscitation

\subsubsection{Brain and muscle oxygen delivery and metabolism during VF and CPR}

A comparison between obtained results in simultaneous brain and biceps femoris muscle measurements are presented in figure 3-2.

Table 2-5 and table 2-6 summarize the induced changes on hemodynamics and different chromophores due to VF and CPR in the muscle and brain of five random animals. All changes were statistically significant $(\mathrm{P}<0.05)$ based on the paired-sample t-test.

The level of significance for the difference between muscle and brain was assessed using tow-sample t-test. Start of the cardiac arrest (VF) induces similar pattern of changes in 
both muscle and brain hemodynamics however as shown in table 2-5, in term of amplitude of the changes there is a statistically significant difference between muscle and brain in $\Delta[\mathrm{HHb}], \Delta[\mathrm{Cyt}-\mathrm{ox}]$ and blood flow (femoral artery vs. carotid) $(\mathrm{p}<0.005)$; [HbO2] drop following VF is not considered statistically different between brain and muscle.

After start of the CPR as shown in table 2-6, the difference between muscle and brain is considered to be statistically significant in $\Delta[\mathrm{HHb}], \Delta[\mathrm{Cyt}-\mathrm{ox}]$ and blood flow (femoral artery vs. carotid $)(\mathrm{p}<0.05)$.

Table 3-3; The impact of VF on different tissue parameters measured by NIRS and hemodynamics (changes are calculated as the changes $120 \mathrm{~s}$ after induction of the VF minus baseline values); Pvalues are calculated between muscle and brain (two sample t-test, two-tailed, 8 degree of freedom)

\begin{tabular}{|c|c|c|c|c|c|c|c|c|c|c|c|}
\hline \multirow[t]{2}{*}{$\begin{array}{c}\text { Case } \\
\text { no. }\end{array}$} & \multicolumn{2}{|c|}{$\begin{array}{c}\Delta\left[\mathrm{HbO}_{2}\right] \\
(\mu \mathrm{M})\end{array}$} & \multicolumn{2}{|c|}{$\begin{array}{c}\Delta[\mathbf{H H b}] \\
(\mu \mathrm{M})\end{array}$} & \multicolumn{2}{|c|}{$\begin{array}{c}\Delta[\text { Cyt-ox }] \\
\quad(\mu M)\end{array}$} & \multicolumn{2}{|c|}{$\begin{array}{c}\Delta \mathrm{tSO}_{2} \\
(\%)\end{array}$} & \multicolumn{2}{|c|}{$\begin{array}{c}\mathrm{BF} \\
\mathrm{ml} / \mathrm{min}\end{array}$} & $\underset{(\mathrm{mmHg}}{\mathrm{BP}}$ \\
\hline & Muscle & Brain & Muscle & Brain & Muscle & Brain & Muscle & Brain & $\mathrm{mFeF}$ & $\mathrm{mCBF}$ & $\mathrm{mAoP}$ \\
\hline 1 & -9.59 & -8.51 & 3.19 & 8.46 & -1.2 & -3.23 & -12.54 & -12.7 & -40.04 & -282.8 & -53.11 \\
\hline 2 & -6.48 & -7.79 & 1.19 & 10.7 & -0.77 & -2.09 & -7.72 & -14.06 & -44.91 & -202.4 & -50.78 \\
\hline 3 & -11.42 & -4.86 & 4.32 & 5.21 & -1.54 & -1.68 & -15.63 & -7.64 & -48.22 & -321.2 & -52.63 \\
\hline 4 & -3.17 & -18.03 & -1.49 & 9.83 & -1.49 & -2.41 & -1.4 & -18.53 & -63.22 & -329 & -69.27 \\
\hline 5 & -6.26 & -4.06 & 3.93 & 5.59 & -1.58 & -2.28 & -10.76 & -6.47 & -52.93 & -209 & -56.78 \\
\hline $\begin{array}{l}\text { Mean } \\
\pm \text { SD }\end{array}$ & $\begin{array}{c}-7.38 \\
2.86\end{array}$ & $\begin{array}{c}-8.65 \\
4.98\end{array}$ & $\begin{array}{l}2.22 \\
2.14\end{array}$ & $\begin{array}{l}7.95 \\
2.21\end{array}$ & $\begin{array}{c}-1.32 \\
0.3\end{array}$ & $\begin{array}{c}-2.34 \\
0.5\end{array}$ & $\begin{array}{c}-9.61 \\
4.8\end{array}$ & $\begin{array}{l}-11.88 \\
4.4\end{array}$ & $\begin{array}{c}-49.86 \\
7.89\end{array}$ & $\begin{array}{c}-268.8 \\
53.94\end{array}$ & $\begin{array}{c}-56.51 \\
6.67\end{array}$ \\
\hline $\mathrm{P}$ & \multicolumn{2}{|c|}{0.63} & \multicolumn{2}{|c|}{0.003} & \multicolumn{2}{|c|}{0.004} & \multicolumn{2}{|c|}{0.45} & \multicolumn{2}{|c|}{0.0001} & - \\
\hline
\end{tabular}

The main difference between muscle and brain is that after the beginning of the resuscitation (CPR or EPI) in the muscle [HbO2] and [HHb] have similar behavior and decreased or increased together; in the brain though [HbO2] and [HHb] changed in opposite directions. 
Table 3-4; The impact of CPR on different tissue parameters measured by NIRS and hemodynamics (changes are calculated as the maximum changes during CPR minus signal amplitude before CPR); $P$-values are calculated between muscle and brain (two sample t-test, two-tailed, 8 degree of freedom)

\begin{tabular}{|c|c|c|c|c|c|c|c|c|c|c|c|}
\hline \multirow[t]{2}{*}{$\begin{array}{c}\text { Case } \\
\text { no. }\end{array}$} & \multicolumn{2}{|c|}{$\begin{array}{c}\Delta\left[\mathrm{HbO}_{2}\right] \\
(\mu \mathrm{M})\end{array}$} & \multicolumn{2}{|c|}{$\begin{array}{c}\Delta[\mathbf{H H b}] \\
(\mu \mathrm{M})\end{array}$} & \multicolumn{2}{|c|}{$\begin{array}{c}\Delta[\text { Cyt-ox }] \\
\quad(\mu M)\end{array}$} & \multicolumn{2}{|c|}{$\begin{array}{c}\Delta \mathrm{tSO}_{2} \\
(\%)\end{array}$} & \multicolumn{2}{|c|}{$\begin{array}{c}\text { BF } \\
\mathrm{ml} / \mathrm{min}\end{array}$} & \multirow{2}{*}{$\begin{array}{c}\text { BP } \\
(\mathbf{m m H g} \\
) \\
\mathrm{mAoP}\end{array}$} \\
\hline & Muscle & Brain & Muscle & Brain & Muscle & Brain & Muscle & Brain & $\mathrm{mFeF}$ & $\mathrm{mCBF}$ & \\
\hline 1 & 2.77 & 2.11 & 1.98 & -0.92 & -0.59 & 1.17 & 2.04 & 1.09 & 41.14 & 70.5 & 17.56 \\
\hline 2 & 3.07 & 5.01 & 1.32 & -2.22 & -0.15 & 0.99 & 1.8 & 5.05 & 21.93 & 44.06 & 28.29 \\
\hline 3 & 1.51 & 2.84 & 0.83 & 1.64 & -0.02 & 0.04 & 0.81 & 0.75 & 0.89 & 79.25 & 11.79 \\
\hline 4 & -0.28 & 1.97 & -0.31 & -1.41 & 0.22 & -0.03 & 0.21 & 1.73 & -0.27 & 24.87 & 20.03 \\
\hline 5 & 3.32 & 2.94 & 6.08 & -1.26 & -1.08 & 0.21 & -2.29 & 1.51 & 17.9 & 41.48 & 24.88 \\
\hline Mean & 2.08 & 2.97 & 1.98 & -0.8 & -0.32 & 0.47 & 0.51 & 2.02 & 16.32 & 20.51 & 52.03 \\
\hline$\pm \mathrm{SD}$ & 1.33 & 1.09 & 2.18 & 1.3 & 0.46 & 0.5 & 1.55 & 1.54 & 15.25 & 5.74 & 19.97 \\
\hline $\mathrm{P}$ & \multicolumn{2}{|c|}{0.24} & \multicolumn{2}{|c|}{0.04} & \multicolumn{2}{|c|}{0.03} & \multicolumn{2}{|c|}{0.2} & \multicolumn{2}{|c|}{0.02} & - \\
\hline
\end{tabular}

In all animals oxidized [Cyt-ox] drops significantly following VF in both muscle and the brain however as it is expected due to greater mitochondrial density in cerebral cells hence higher concentration of cytochrome oxidase in the brain, the average $\Delta[$ Cyt-ox $]$ following VF in the brain is greater than muscle $(\mathrm{p}<0.005)$.

Although in the muscle [HbO2] and [HHb] change in the same direction following $\mathrm{CPR}$, in some animals due to greater changes in [HbO2], tissue oxygen saturation ( $\mathrm{tSO} 2)$ follows [HbO2] and oxidized [Cyt-ox] follows [HbO2] as well.

Start of the CPR results in an increase in the $\mathrm{tSO} 2$ and oxidized [Cyt-ox] in the brain.

The effect of CPR on enhancement of oxidized [Cyt-ox] is much more significant in the brain than muscle $(\mathrm{p}<0.05)$. 
While CPR is still in process each epinephrine (EPI) bolus administration enhances the aortic blood pressure (AoP), carotid blood flow (CBF) and femoral artery blood flow (FeF) approximately $10 \mathrm{~s}$ following the injection.

When EPI bolus was administered during CPR both $[\mathrm{HbO} 2]$ and $[\mathrm{HHb}]$ in the muscle increased $(\mathrm{p}<0.05)$. In the cerebral tissue EPI increases [HbO2] and decreases in $\mathrm{HHb}$. The increased oxygenation due to EPI was observed in the muscle prior to that in the brain reflecting a faster response to EPI in the muscle compared to brain.

In non-survival animals in the muscle EPI causes a transient sharp reduction (average 0.2 $\mu \mathrm{M})$ in oxidized [Cyt-ox] (15-20 s) concurrent with the induced rapid enhancement in [HbO2] however no significant changes was observed in the brain due to EPI; in survival animals [Cyt-ox] is completely mimicking the $\mathrm{tSO} 2$ changes. The $\mathrm{HbO} 2$ and $\mathrm{HHb}$ changes in the muscle tissue to the EPI boluses are ahead of tissue by few seconds. 

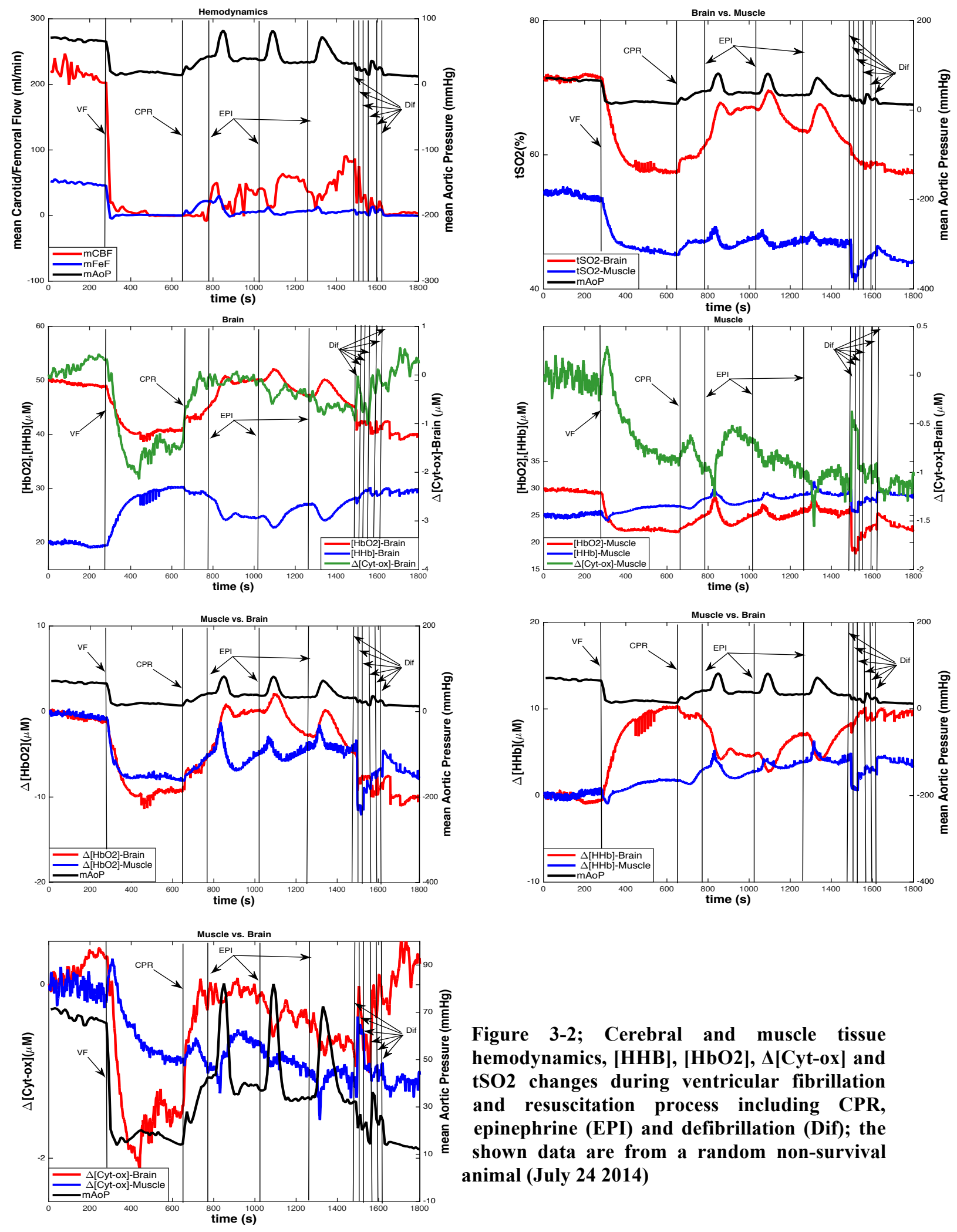

Figure 3-2; Cerebral and muscle tissue hemodynamics, [HHB], [HbO2], $\Delta[\mathrm{Cyt}-\mathrm{Ox}]$ and tSO2 changes during ventricular fibrillation and resuscitation process including CPR, epinephrine (EPI) and defibrillation (Dif); the shown data are from a random non-survival animal (July 24 2014) 


\subsubsection{Brain oxygenation and metabolism in survival vs. non-survival animals}

Figure 3-3 and figure 3-4 show sample results of two random pairs of survival and nonsurvival animals with similar resuscitation protocols to compare hemodynamics, oxygen delivery and metabolism level during cardiac arrest and resuscitation in a survival case vs. a non-survived case.

The first pair of animals (Fig.3-3) remained untreated under cardiac arrest for approximately 5 min followed by CPR and defibrillation. In both survival and nonsurvival animals $[\mathrm{HbO} 2]$ declines and $[\mathrm{HHb}]$ rises significantly simultaneously along with a huge drop in AoP. Also in both animals CPR was very effective in recovering brain oxygen delivery and $\mathrm{tSO}$, which was accompanied by significant drop in [HHb] level. In the survival animal oxidized [Cyt-ox] was not fully recovered yet with CPR although due to the early defibrillation it is fully recovered along with tSO2 .In nonsurvival animal though longer CPR recovered both oxidized [Cyt-ox] and tSO2 level however when CPR was stopped and defibrillations were applied both oxidized [Cyt-ox] and $\mathrm{tSO} 2$ declined after each shock and eventually the animal died.

In the second sample pair of animals (Fig.3-4) both remained in VF for 6 min and resuscitation protocols contained CPR, defibrillation and EPI. In both survival and nonsurvival animals as it was expected induction of VF resulted in a significant drop in AoP, $\mathrm{CBF}, \mathrm{FeF}, \mathrm{tSO} 2,[\mathrm{HbO} 2]$ and oxidized [Cyt-ox]. CPR was effective in both animals resulting in enhancement of $\mathrm{AoP}, \mathrm{CBF}$ and $[\mathrm{HbO} 2]$ and reduction of [HHb]. EPI infusion during $\mathrm{CPR}$ in both animals caused a sharp increase in AoP and [HbO2] accompanied by growing oxidized [Cyt-ox] and tSO2 in survived animal. 
In non-survival animals in the muscle EPI causes a transient sharp reduction (average 0.2 $\mu \mathrm{M})$ in oxidized [Cyt-ox] (15-20 s) concurrent with the induced rapid enhancement in [HbO2] however no significant changes was observed in the brain due to EPI; in survival animals [Cyt-ox] is perfectly mimicking the tSO2 changes due o EPI.

The main difference between a survival and non-survival animal is the behavior of the [Cyt-ox] redox state. In the survival animal [Cyt-ox] changes is well correlated with tSO2 and it is not fully recovered even after successful defibrillation, however in non-survival animal the [Cyt-ox] redox level is fully recovered but not affected by tSO2. It may be due to malfunction of the citric acid cycle or glycolysis process by which the reducing agents are generated to trigger the electron transport chain in the inner mitochondrial membrane; this means that no electron is transported to the cytochrome $\mathrm{c}$ hence cyt-ox will remain oxidized and reduced enzyme is minimized. 

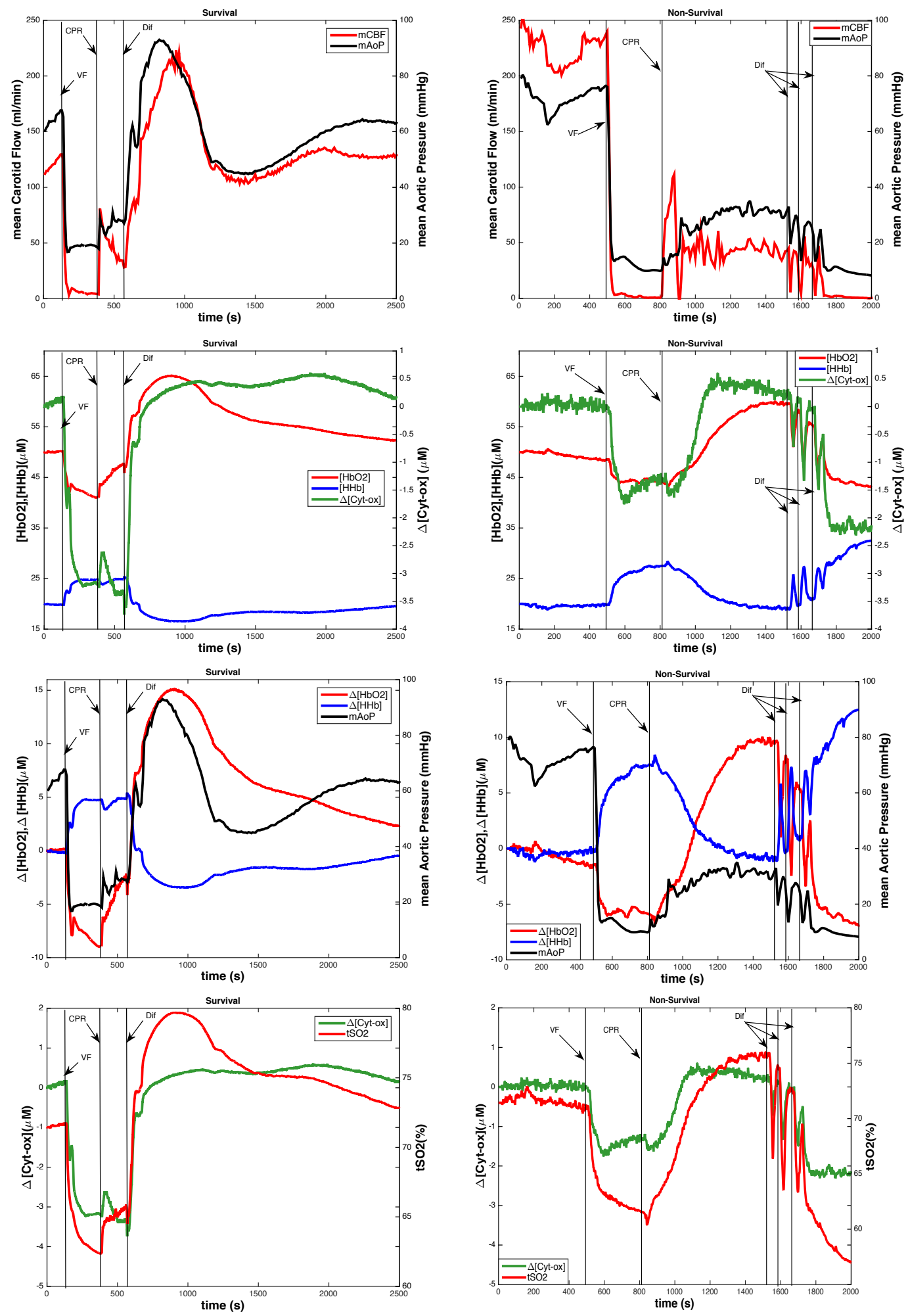

Figure 3-3; Comparing a survival case vs. a non-survival case; hemodynamics and NIRS measured cerebral $[\mathrm{HHB}],\left[\mathrm{HbO}_{2}\right], \Delta[\mathrm{Cyt}-\mathrm{ox}]$ and $\mathrm{tSO}_{2}$ changes during ventricular fibrillation and resuscitation 

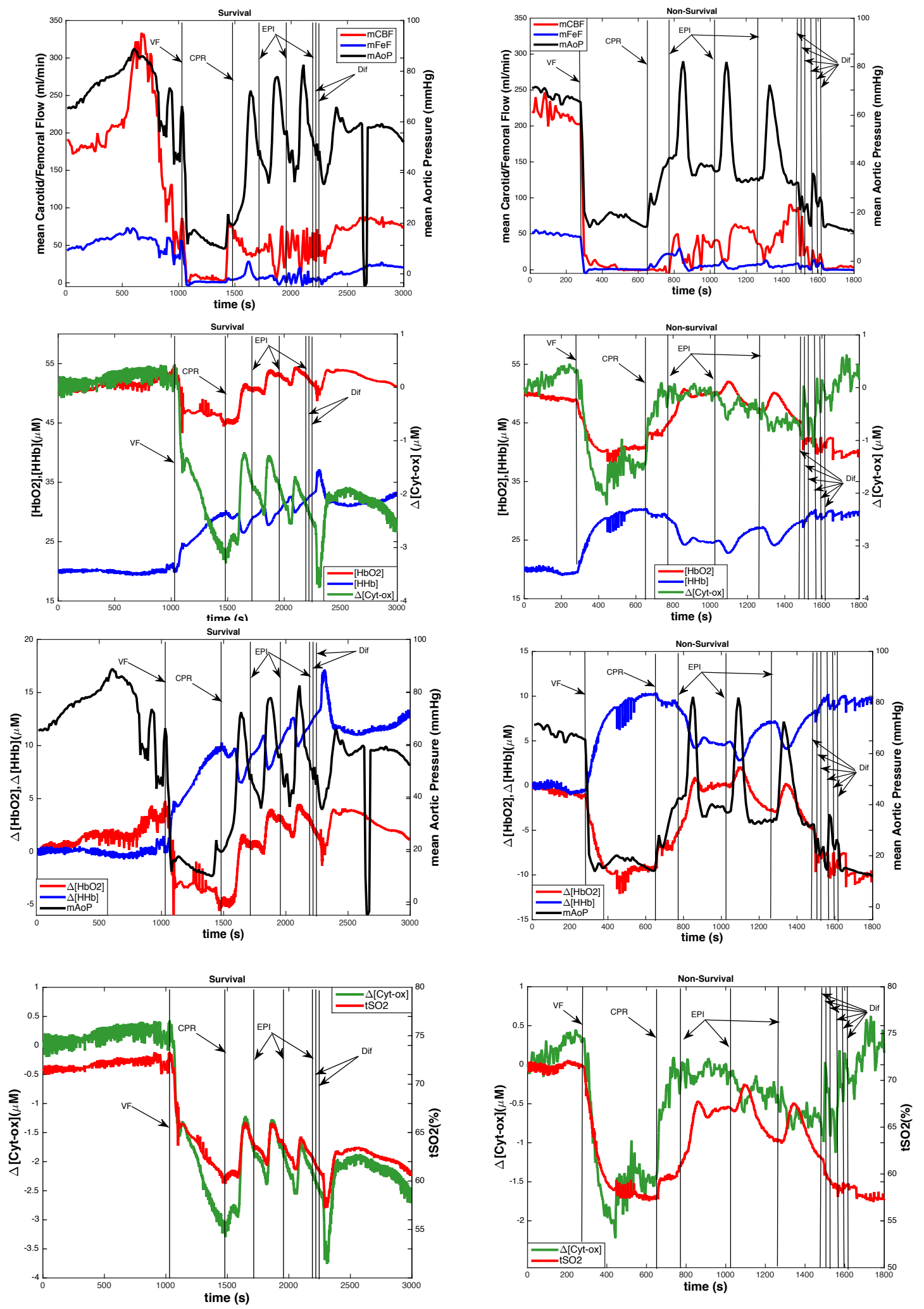

Figure 3-4; Comparing a survival (plots on left column) case vs. a non-survival (plots on the right column) case; hemodynamics and NIRS measured cerebral $[\mathrm{HHB}],\left[\mathrm{HbO}_{2}\right], \Delta[\mathrm{Cyt}-\mathrm{ox}]$ and $\mathrm{tSO}_{2}$ changes during ventricular fibrillation and resuscitation 


\subsubsection{The effect of rapid pacing on cardiac output, cerebral oxygenation and metabolism}

Figure 3-5 shows the results of three different pacing rates $(150,200$ and $250 \mathrm{bpm})$ on hemodynamic parameters including AoP, CBF, FeF and NIRS measured cerebral oxygen delivery and metabolism. Table 2-7 summarizes the induced changes due to alternation of pacing rate on cerebral hemodynamics and metabolism.

As expected heart rate in inversely proportional to the cardiac output. AoP and consequently $\mathrm{CBF}$ and FeF decline dramatically immediately after increasing the pacing rate temporarily. The higher the pacing rate the greater fall in hemodynamics is observed. $\left[\mathrm{HbO}_{2}\right]$ and oxidized [Cyt-ox] decline with AoP and [HHb] rises following rapid pacing. A sharp decline in hemodynamics is observed following onset of the rapid pacing, baseline levels are almost recovered after stopping the rapid pacing. The recovery time as are shown in table $2-7$, differ in different parameters. The fastest recovery belongs to cerebral $\mathrm{HbO}_{2}$ level and the latest recovery is for aortic pressure (AoP) and femoral artery blood flow (FeF). In NIRS measured parameters first $\mathrm{HbO}_{2}$ returns to normal level and then $\mathrm{HHb}$ with average 10s delay is recovered and finally oxidase cyt-ox is regained after average $2 \mathrm{~s}$.

Similar to hemodynamics brain oxygen delivery and metabolism is inversely proportional to pacing rate however the decline in hemodynamics is steeper than oxygenation and metabolism level but the recovery to the normal level is faster in cerebral oxygenation and metabolism level than of that in cardiac output. 
According to the paired-sample t-test (two-tailed, 11 degree of freedom) different pacing rates induce statistically significant different changes in both cerebral and muscle hemodynamics $(\mathrm{p}<0.005)$.

Carotid blood flow is regained approximately 10s ahead of femoral flow and aortic pressure reflecting cerebral autoregulation that could be due to vasodilation hence reducing the resistance of brain feeding vasculature including carotid.

Table 3-5; The impact of different pacing rate on cerebral hemodynamics and metabolism; $\Delta \mathrm{P} / \Delta \mathrm{F} / \Delta \mathrm{C}$ represent the maximum drop in blood pressure/flow or concentration from the baseline value due to changing pacing rate and $\Delta t$ represents the recovery time from the onset of each pacing rate to the initial value

\begin{tabular}{|c|c|c|c|c|c|c|c|c|c|c|c|c|}
\hline \multirow{2}{*}{$\begin{array}{c}\text { Pacing } \\
\text { Rate } \\
\text { (bpm) }\end{array}$} & \multicolumn{2}{|c|}{ AoP } & \multicolumn{2}{|c|}{$\mathrm{CBF}$} & \multicolumn{2}{|c|}{$\mathrm{FeF}$} & \multicolumn{2}{|c|}{$\mathbf{H b O}_{2}$} & \multicolumn{2}{|c|}{ HHb } & \multicolumn{2}{|c|}{ Cyt-ox } \\
\hline & $\mathrm{mmHg}$ & Sec. & $\mathrm{ml} / \mathrm{min}$ & Sec. & $\mu \mathrm{M}$ & $\mathrm{Sec}$ & $\mu \mathrm{M}$ & Sec. & $\mu \mathrm{M}$ & Sec. & $\mu \mathrm{M}$ & Sec. \\
\hline & $\Delta \mathbf{P}$ & $\Delta t$ & $\Delta \mathbf{F}$ & $\Delta t$ & $\Delta \mathbf{F}$ & $\Delta \mathbf{t}$ & $\Delta \mathrm{C}$ & $\Delta t$ & $\Delta \mathrm{C}$ & $\Delta t$ & $\Delta \mathrm{C}$ & $\Delta t$ \\
\hline 150 & -24.6 & 220 & -102.6 & 210 & -49.16 & 220 & -0.24 & 181 & 3.96 & 189 & -0.71 & 190 \\
\hline 200 & -36.26 & 140 & -152.5 & 130 & -64.12 & 140 & -8.76 & 99.2 & 7.18 & 110 & -2.06 & 112 \\
\hline 250 & -40.1 & 160 & -166.4 & 150 & -68.1 & 160 & -11.9 & 124 & 8.69 & 137 & -2.44 & 141 \\
\hline
\end{tabular}



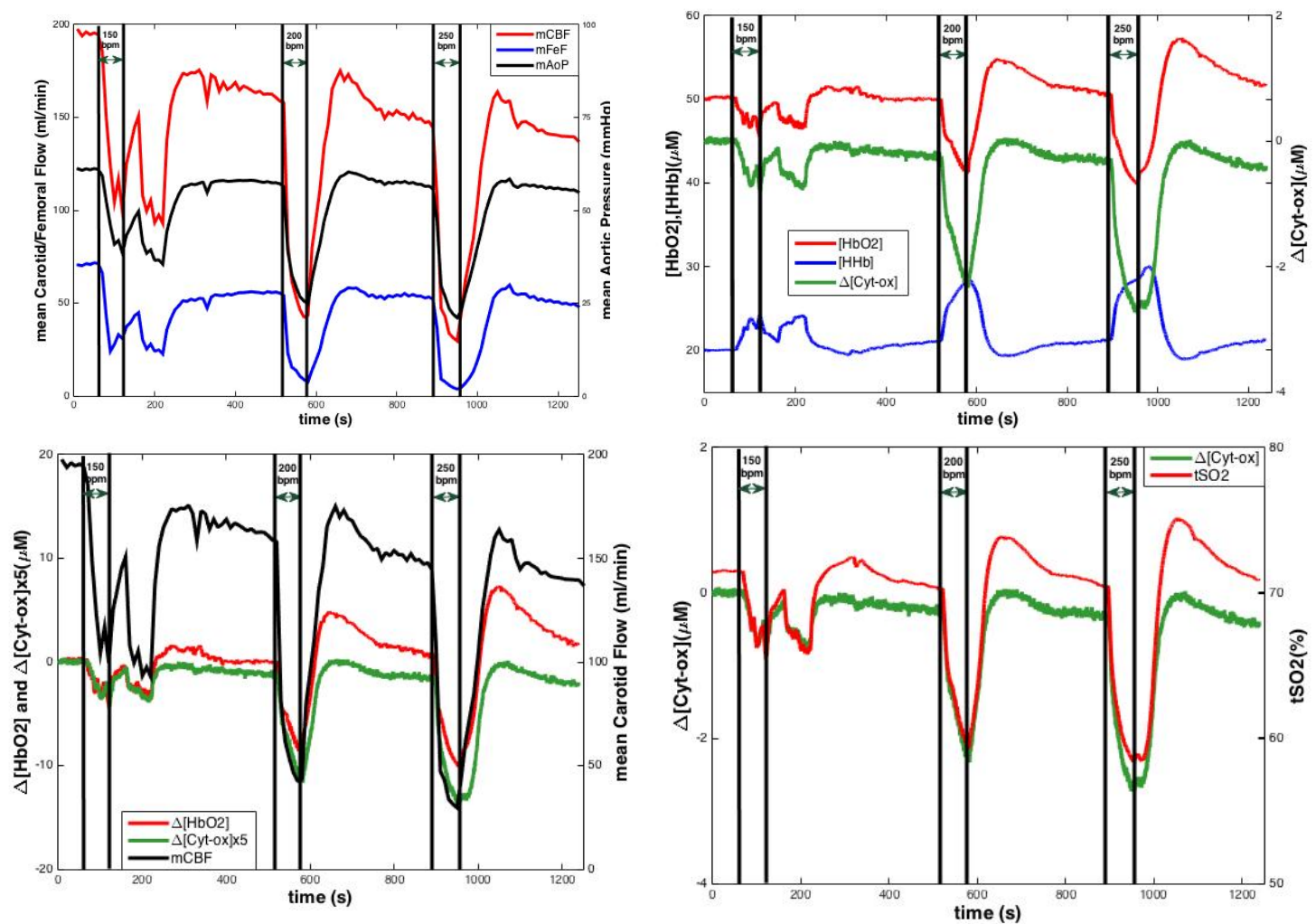

Figure 3-5; The effect of different pacing rates on hemodynamics and IIRS measured cerebral oxygenation and metabolism level

\subsubsection{The effect of $\mathrm{FiO}_{2}$ on cardiac output, cerebral oxygenation and metabolism}

Figure 3-6 shows the results of applying different inhaled oxygen fraction including $30 \%$ and $14 \%$ respectively. Increasing inhaled O2 level from normal atmospheric level of $21 \%$ to $30 \%$ slightly reduces both [HbO2] and [HHb] due to vasoconstriction, inducing minor changes in cerebral oxygen saturation though $(\approx 1 \%)$; In addition it enhanced cerebral oxygen metabolism reflected by growing oxidized [Cyt-ox] level by $0.68 \mu \mathrm{M}$; this is due to increased oxygen level in the blood both in the RBCs and plasma.

Reducing inhaled oxygen concentration to $14 \%$ induced a transient enhancements in [HbO2], oxygen saturation tSO2 and oxidized [Cyt-ox] concentration while reducing 
$[\mathrm{HHb}]$ however eventually low oxygen saturation resulted in a significant drop in both oxygenation and metabolism indices $(\mathrm{p}<0.005)$ in the brain. It seems that poor oxygenation in the brain triggers a regulatory system that tries to maintain cerebral oxygenation by vasodilation that resulted in those temporary rises however it eventually fails.

FiO2 alternations resulted in similar and continuous changes in hemodynamic. Both increasing and decreasing inhaled oxygen saturation to $30 \%$ and $14 \%$ improved $\mathrm{mAoP}$, $\mathrm{CBF}$ and FeF. Table 2-8 represents all induced changes by $\mathrm{FiO} 2$ changes.
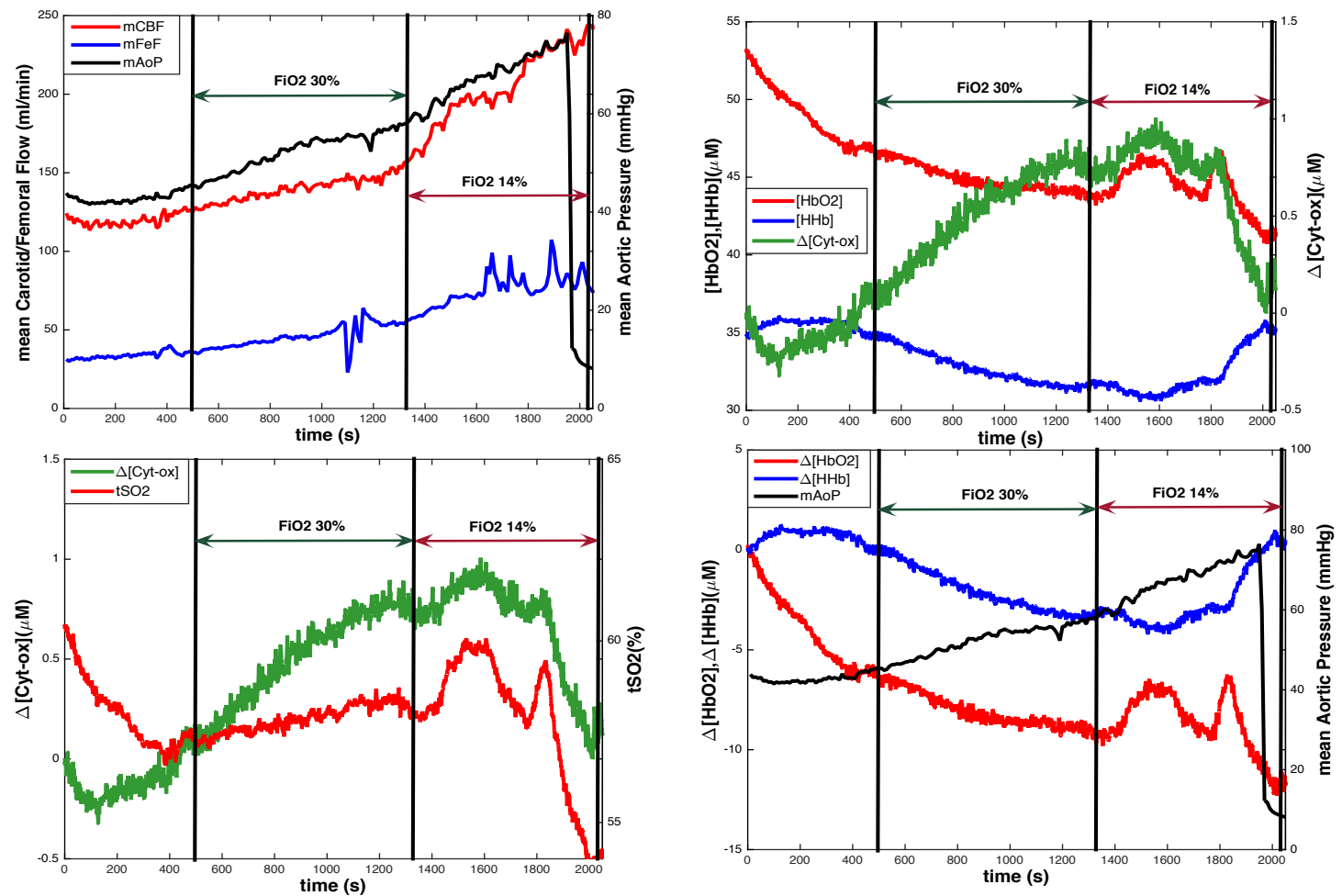

Figure 3-6; The effect of $\mathrm{FiO2}$ alternation (30\% and $14 \% \mathrm{O} 2$ respectively) on hemodynamics and NIRS measured cerebral oxygenation and metabolism level 
Table 3-6; The effect of $\mathrm{FiO}_{2}$ alternation on hemodynamics and cerebral oxygenation and metabolism. The changes are calculated as the difference between the first and last moment of inhaled $\mathrm{O}_{2}$ level.

\begin{tabular}{|c|c|c|c|c|c|c|c|}
\hline $\begin{array}{c}\mathbf{O}_{2} \\
\%\end{array}$ & $\begin{array}{c}\Delta\left[\mathrm{HbO}_{2}\right] \\
(\mu \mathrm{M})\end{array}$ & $\begin{array}{c}\Delta[\mathbf{H H b}] \\
(\mu \mathrm{M})\end{array}$ & $\begin{array}{c}\Delta[\text { Cyt-ox }] \\
(\mu M)\end{array}$ & $\begin{array}{c}\Delta \mathrm{tSO}_{2} \\
\%\end{array}$ & $\begin{array}{c}\Delta(\mathrm{mAoP}) \\
(\mathrm{mmHg})\end{array}$ & $\begin{array}{c}\Delta(\mathrm{mCBF}) \\
(\mathrm{ml} / \mathrm{min})\end{array}$ & $\begin{array}{l}\Delta(\mathrm{mFeF}) \\
(\mathrm{ml} / \mathrm{min})\end{array}$ \\
\hline 30 & -3.2 & -2.93 & 0.68 & 1.05 & 12.59 & 30.3 & 18.51 \\
\hline 14 & -2.43 & 3.62 & -0.63 & -4.01 & 16.35 & 80.7 & 31.6 \\
\hline
\end{tabular}

\subsubsection{Conclusion and Discussion}

In this series of animal experiments on resuscitation after cardiac arrest we had a unique opportunity to measure hNIRS parameters directly on the brain surface (dura), or on the brain through the intact skull, and, in some experiments, also to acquire simultaneously muscle hNIRS data. Simultaneously the aortic blood pressure and carotid and femoral blood flow were recorded in real time using invasive sensors. The comparison of hNIRS data acquired on brain surface and through the adult pig skull shows that in both cases $\Delta[\mathrm{HbO} 2], \Delta[\mathrm{HHb}]$, and $\Delta[\mathrm{ox}-\mathrm{CCO}]$ changed in similar ways in similar situations and in agreement with pressure and flow changes. The comparison of simultaneously measured brain and muscle changes showed expected differences. Temporal changes in $\Delta[\mathrm{HbO}]$, $\Delta[\mathrm{HHb}]$, and $\Delta[\mathrm{ox}-\mathrm{CCO}]$ strongly depended on the assumed baseline optical properties, mostly on the wavelength-dependent reduced scattering coefficient. Since hNIRS can measure both baseline properties and changes [15], hNIRS may be a better concept than mNIRS. Overall the research shows feasibility of the hNIRS for measurements of cerebral parameters in human cardiac arrest patients. Further research will be directed on revealing the role of Epinephrine infusions and electric shocks during resuscitations, and development of a clinical portable hNIRS system. 


\section{Chapter 4}

\section{Discussion, Conclusion and Future Work}

\subsection{Discussion}

In this study the main objective was to improve hyperspectral NIRS for both functional and non-functional studies. Different algorithms were designed for processing NIRS data to improve the deconvolution of the Cyt-ox signal as an intracellular index for oxygen metabolism level.

In both functional and non-functional measurements we employed CW hyperspectral (broadband) NIRS with spectral range of 650-1100 nm.

In the first part of this thesis the event-related changes in the activation pattern and metabolism in the frontal lobe of the brain were measured. Relative changes in $\mathrm{HbO}_{2}$, $\mathrm{HHb}$ and Cyt-ox redox state were calculated using different algorithms.

Based on the statistical analysis on the results the algorithm that employs ICA and diffusion approximation and performs least-square curve fit is the best approach among all examined algorithms. Similar experiments were performed using fMRI and the fNIRS results were validated with fMRI results; all event-related activation or deactivations patterns observed in fNIRS were well correlated with those of fMRI. 
Our results show that MBLL and diffusion approximation obtain very similar results for changes in $\mathrm{HbO}_{2}$ and $\mathrm{HHb}$, however Cyt-ox redox changes calculated by diffusion approximation yield much greater statistical significance and consistency; MBLL though performs unreliably in calculation of Cyt-ox changes. In addition for hyperspectral functional NIRS studies addition of the ICA to the processing significantly improves the consistency of the calculated Cyt-ox signal. As opposed to previous studies by Toronov et al. [31] instead of using the individual components calculated by ICA as source signals, in this study after centering and reducing the dimensions of the data FastICA was implemented then calculated independent components were combined and were backtransformed into the time spectral domain. The main application of the FastICA in this study was removing the Gaussian sources that are considered as noise and de-noise the data. After FastICA and back-transforming the data the absorbance was modeled with diffusion approximation.

In addition as we reduced the dimensions of the signal to five and performed ICA, the correlation between the calculated independent component and concentrations changes calculated by diffusion approximation was investigated and it was observed that cyt-ox signal calculated by diffusion model is not correlated with any of the components, $\mathrm{HbO}_{2}$ and $\mathrm{HHb}$ though were partially correlated with two of the components. This may be due to the nature of the Cyt-ox signal, which is the difference spectrum between oxidized and reduced states and is not directly distinguishable from other signal sources and requires further processing and employing the corresponding extinction spectrum.

In the second part of my research in a series of extremely unique and sophisticated studies, brain activity was monitored during cardiac arrest and resuscitation. In addition 
to hyperspectral NIRS, Aortic blood pressure, carotid flow and femoral artery flow were monitored invasively to investigate the correlation between results.

One of the most interesting features that was very interesting was the comparison between the measured signal through the skull vs. the signal that obtained invasively by probing the brain over the dura mater. All the experiments were performed on adult pigs with non-negligible skull thickness. Both types of measurements showed similar changes in terms of time course of the signal; the amplitude of the changes were different though. The hemodynamic parameters (aortic blood pressure and carotid blood flow) were well correlated with NIRS measured changes both invasively and semi-invasively.

These results are very important as the validity of the non-invasively NIRS measured changes through the skull (with adult skull thickness), were confirmed in terms of time course of the signal.

Comparison between survival and non-survival animals revealed there is no statictically significant difference between NIRS measured chromophores behavior during resuscitation, that could differentiate between brain response in survival vs. non survival animal.

In these experiment to investigate the hemodynamic responses in the brain vs. muscle, NIRS signal was simultaneously recorded on the muscle and cerebral tissue. Smaller changes in the muscle were observed specially in Cyt-ox level. As expected since the mitochondrial density is greater in the cerebral cells, the concentration of the Cyt-ox is greater and consequently NIRS measured changes are greater in the brain than the muscle. One other interesting finding was that although sometimes CPR results in an overshoot in $\mathrm{HbO}_{2}$ the Cyt-ox barely exceeds the reference level. In addition the behavior 
of the Cyt-ox explains the cellular metabolism level, which is quite different in the muscle and the brain during resuscitation.

The perfect correlation between hemodynamic parameters that were measured invasively with NIRS measured parameters proves the reliability of the NIRS.

Our novel signal-processing algorithm significantly improved the reliability of the calculated Cyt-ox by employing diffusion approximation, stepwise approach and first derivative of the spectral extinction coefficient. Our algorithm minimized the cross-talk of different chromophores by minimizing the overlapping spectral band of Cyt-ox and other chromophores.

In addition all of the results revealed that Cyt-ox is well correlated with tissue oxygen saturation rather than $\mathrm{HbO}_{2}$ or $\mathrm{HHb}$ as opposed to other studies.

One of the main concerns during cardiac arrest is the brain damage that results in cell death or reduced oxygen metabolism; in some survived animals it was seen that although the animal survived and $\mathrm{HbO}_{2}$ returned to the reference level, Cyt-ox as well as tissue oxygen saturation were not recovered that may reflect brain cell damage. This is another signature of the Cyt-ox signal that shows more brain specificity than hemoglobin.

Different resuscitation protocols were assesses during 19 experiments. In six experiments the CPR was accompanied by epinephrine (EPI) bolus injection that induced a transient effect on enhancement of oxygen delivery and metabolism. There was no evidence of increasing in survival rate due to administration of the EPI. 


\subsection{Conclusion}

Hyperspectral CW NIRS has shown to be very useful technique for quantification of the tissue chromophores. Monitoring intracellular oxygen metabolism through real time measurement of redox cytochrome oxidase is the most unique feature of the NIRS that can be only obtained through hyperspectral (broadband) systems. Measurement of low concentration chromophores such as Cyt-ox and decontaminating the signal from physiological noise and cross talk with high concentration chromophores $\left(\mathrm{HbO}_{2}\right.$ and $\mathrm{HHb}$ ) is a very challenging process that can be achieved only through hyperspectral systems. Heperspectral NIRS provides very high spectral resolution that enables implementation of several signal-processing algorithms for deconvolution of different chromophores. Although hyperspectral CW NIRS systems are relatively inexpensive, they provide higher signal to noise ratio compared to time domain and frequency domain systems therefor they have higher potential to measure low concentration substances.

Our results showed that independent component analysis (ICA) is a very effective step for de-noising the functional NIRS data to be prepared for modeling the absorbance to better resolve Cyt-ox changes. In addition we showed that diffusion approximation theory for a semi-infinite medium and employing a step-wise algorithm using the first derivative of the absorbance spectra along with least-square curve fitting is very useful for calculation of the redox changes of Cyt-ox. Modeling of absorption based on MBLL requires calculation of DPF, which depends on different factors including wavelength, however in CW NIRS studies DPF is assumed to be constant, diffusion model though is independent of DPF therefore for smaller concentration changes such as Cyt-ox results in more consistent and reliable values. 
In this research project reliability of the hyperspectral NIRS in functional studies was assessed and validated with fMRI. In addition the ability of the non-invasive NIRS measurements during cardiac arrest and resuscitation was approved.

\subsection{Future work}

We propose to apply in the future the signal-processing algorithm introduced here to in functional studies for monitoring brain activity as a diagnostic technique especially for concussion patients. 


\section{References}

[1] FF Jobsis, "Noninvasive, infrared monitoring of cerebral and myocardial oxygen sufficiency and circulatory parameters," Science, no. 198, pp. 1264-1267, 1977.

[2] D. Boas and M.A. Franceschini, "Near infrared imaging," Scholarpedia, no. 4, p. 6997, 2009.

[3] M. Ferrari, I. Giannini, G. Sideri, and E. Zanette, "Continuous non invasive monitoring of human brain by near infrared spectroscopy," Advances in experimental medicine and biology , no. 191, pp. 873-882, 1985.

[4] D. T. Delpy et al., "Estimation of optical pathlength through tissue from direct time of flight measurement," Physics in Medicine and Biology, no. 33, pp. 14331442, 1988.

[5] F. H. Schlereth, J. M. Fossaceca, A. D. Keckler, and R. L. Barbour, "Multicomputer-based neural networks for imaging in random media," in IEEE Nuclear Science Symposium and Medical Imaging Conference, 1991, p. 2193.

[6] B. Chance, Z. Zhuang, C. Unah, C. Alter, and L. Lipton, "Cognition-activated lowfrequency modulation of light absorption in human brain," in National Academy of Sciences of the United States of America , 1993, pp. 3770-3774.

[7] A. Villringer, J. Planck, C. Hock, L. Schleinkofer, and U. Dirnagl, "Near infrared spectroscopy (NIRS): A new tool to study hemodynamic changes during activation of brain function in human adults," Neuroscience Letters, no. 154, pp. 101-104, 1993.

[8] Mitra S., Meek J., Robertson N., Tachtsidis I. Bale G., "A new broadband nearinfrared spectroscopy system for in-vivo measurements of cerebral cytochrome-c-oxidase changes in neonatal brain injury," Biomed Opt Express., pp. 3450-3466, 2014.

[9] Elwell C. E., Cooper C. E., Cope M., Delpy D. T. Matcher S. J., "Performance comparison of several published tissue near-infrared spectroscopy algorithms," Anal. Biochem. , vol. 227(1), pp. 54-68, 1995.

[10] J J Macklin, J K Trautman, T D Harris, and L E Brus, "Imaging and time-resolved spectroscopy of single molecules at an interface," Science, no. 5259, p. 255, 1996.

[11] Takasaki S., Ozaki T., Kobayashi Y. Suzuki S., "A Tissue Oxygenation Monitor using NIR Spatially Resolved Spectroscopy," in BiOS'99 International Biomedical Optics Symposium. International Society for Optics and Photonics, 1999.

[12] KU Leuven. (2011) Faculteit BIW. [Online]. https://www.biw.kuleuven.be/biosyst/mebios/biophotonicsgroup/fundamental-research/spatially-resolved-spectroscopy

[13] Ruiz Carmona MT, Rubio JJ, de Andrés S Dominguez de Villota ED, "quality of the in vivo and in vitro oxygen-binding capacity of haemoglobin in patients with severe respiratory disease," Br J Anaesth, no. 53, pp. 1325-8, 1981. 
[14] Linda S. Costanzo, Physiology. Hagerstwon, MD.: Lippincott Williams \& Wilkins, 2007.

[15] Salway J., Medical biochemistry at a glance.: John Wiley \& Sons Ltd., 2012.

[16] Jacquez J., Respiratory Physiology.: McGraw-Hill. , 1979.

[17] Murkin J. M. and Arango M., "Near-infrared spectroscopy as an index of brain and tissue oxygenation," Br. J. Anaesth., no. 103, pp. i3-i13, 2009.

[18] Karp G., Cell and Molecular Biology.: John Wiley \& Sons, 2008.

[19] A. Sassaroli and S. Fantini, "Comment on the modified beer-lambert law for scattering media," Physics in Medicine and Biology, no. 14, pp. N255-N257, 2004.

[20] A Beer, "Bestimmung der Absorption des rothen Lichts in farbigen Flüssigkeiten (Determination of the absorption of red light in colored liquids), ," Annalen der Physik und Chemie, vol. 86, pp. 78-88, 1852.

[21] J.H. Lambert, "Photometria sive de mensura et gradibus luminis, colorum et umbrae [Photometry, or, On the measure and gradations of light, colors, and shade]," Augsburg, Augusta Vindelicorum, p. 391, 1760.

[22] Cope M., Van Der Zee P., Arridge S., Wray S., and Wyatt J. Delpy D. T., "Estimation of optical path length through tissue from direct time of flight measurement," Physics in Medicine and Biology, vol. 33, pp. 1433-1442, 1988.

[23] P Herman and A Eke L Kocsis, "The modified Beer-Lambert law revisited," Phys. Med. Biol. , vol. 51, p. 91, 2006.

[24] LV Wang and HI Wu, Biomedical Optics.: John Wiley \& Sons, 2007.

[25] M Franceschini S Fantini, "Semi-infinite geometry in boundary problem for light migration in highly scattering media," J. Opt. Soc. Am, vol. 11, pp. 10-17, 1994.

[26] RC Haskell et al., "Boundary conditions for the diffusion equation in radiative transfer ," Journal of the Optical Society of America, no. 10, pp. 2727-2741, 1994.

[27] LV Wang and SL Jacques, "Sources of error in calculation of optical diffuse reflectance from turbid media using diffusion theory," Computer Methods and Programs in Biomedicine, vol. 61, no. 3, pp. 163-170, 2000.

[28] D A Boas, "DIFFUSE PHOTON PROBES OF STRUCTURAL AND DYNAMICAL PROPERTIES OF TURBID MEDIA," University of Pennsylvania, DISSERTATION 1996.

[29] V Toronov H Yeganeh, "Broadband continuous-wave technique to measure baseline values and changes in the tissue chromophore concentrations," Biomedical Optics Express, vol. 3, p. 2761, 2012.

[30] P Zee, "MEASUREMENT AND MODELLING OF THE OPTICAL PROPERTIES OF HUMAN TISSUE IN THE NEAR INFRARED ," University College London , Dissertation 1992.

[31] I Schelkanova and V Toronov, "Independent component analysis of broadband near-infrared spectroscopy data acquired on adult human head," Biomed Opt 
Express, vol. 3, pp. 64-74, 2012.

[32] J Karhunen, and E Oja A Hyvarinen, Independent component analysis. Adaptive and learning systems for signal processing, communications, and control.: J Wiley \& Sons, 2001.

[33] J Stone, Independent component analysis: a tutorial introduction.: Bradford Books, MIT Press, 2004.

[34] Irina Schelkanova, "Development of Signal Processing for the Broadband Near Infrared Spectroscopy," Ryerson University, Dissertation 2011.

[35] J. HERAULT, C. JUTTEN B. Ans, "Adaptive neural architectures: Detection of primitives," in COGNITIVA'85, 1985, pp. 593-597.

[36] P Comon, "Independent Component Analysis, A New Concept? ", Signal Processing, vol. 36, no. 3, pp. 287-314, 1994.

[37] A. J. Bell and T. J. Sejnowski, "An information-maximization approach to blind separation and blind deconvolution," Neural Comput, vol. 7, pp. 1129-59.

[38] A Belouchrani and A Cichocki, "Robust whitening procedure in blind source separation context," Electronics Letters, vol. 36, pp. 2050-2053, 2000.

[39] Cope M, Springett R, Amess PN, Penrice J, Tyszczuk L, Punwani S, Ordidge R, Wyatt J, Delpy DT Cooper CE, "Use of mitochondrial inhibitors to demonstrate that cytochrome oxidase near-infrared spectroscopy can measure mitochondrial dysfunction noninvasively in the brain," J Cereb Blood Flow Metab., vol. 19, no. 1, pp. 27-38, 1999.

[40] Kohl M, Obrig H, Wenzel R, von Pannwitz W, Matcher SJ, Dirnagl U, Cooper CE, Villringer A Heekeren HR, "Noninvasive assessment of changes in cytochrome-c oxidase oxidation in human subjects during visual stimulation," J Cereb Blood Flow Metab., vol. 19, no. 6, pp. 592-603, 1999.

[41] V Toronov, et al H Zabihi, "Broadband continuous-wave technique to measure baseline values and changes in the tissue chromophore concentrations," Biomedical Optics Express, vol. 3, no. 11, pp. 2761-70, 2012.

[42] Ko KR, Mori S, Winn HR Ngai ALC, "Effect of sciatic nerve stimulation on pial arterioles in rats.," Am J Physiol Heart Circ Physiol, vol. 254, pp. H133-H139, 1988.

[43] Wei L, Otsuka T, Acuff V, Pettigrew K, Patlak C, Fensternmacher Bereczki D, "Hypoxia increases velocity of blood flow through parenchymal microvascular systems in rat brain," J Cereb Blood Flow Metab , vol. 13, pp. 475-486, 1993.

[44] N Kobayashi, M Tamura Y Hoshi, "Interpretation of near-infrared spectroscopy signals: a study with a newly developed perfused rat brain model," Journal of Applied Physiology, vol. 90, no. 5, pp. 1657-1662, 2001.

[45] Raichle ME Fox PT, "Focal physiological uncoupling of cerebral blood flow and oxidative metabolism during somatosensory stimulation in human subjects," Proc Natl Acad Sci USA, vol. 83, pp. 1140-1144, 1986.

[46] Ghosh A, Tachtsidis I, Highton D, Cooper CE, Smith M, Elwell CE. Kolyva C, "Cytochrome c oxidase response to changes in cerebral oxygen delivery in the 
adult brain shows higher brain-specificity than haemoglobin," neuroimage, vol. 85, no. 1, pp. 234-44, 2014.

[47] Martin M. Tisdall, Caroline Pritchard, Terence S. Leung, Arnab Ghosh , Clare E. Elwell , Martin Smith Ilias Tachtsidis, "Analysis of the Changes in the Oxidation of Brain Tissue Cytochrome c Oxidase in Traumatic Brain Injury Patients during Hypercapnoea: a Broadband NIRS Study," Adv Exp Med Biol., pp. 701:9-14, 2011.

[48] Martin M. Tisdall, Ilias Tachtsidis, Terence S. Leung, Clare E. Elwell, and Martin Smith, "Near-infrared spectroscopic quantification of changes in the concentration of oxidized cytochrome c oxidase in the healthy human brain during hypoxemia," Journal of Biomedical Optics, vol. 12, no. 2, 2007.

[49] Jöbsis FF., "Noninvasive, infrared monitoring of cerebral and myocardial oxygen sufficiency and circulatory parameters.," Science., pp. 198:1264-1267, 1977.

[50] Smith M., "hedding light on the adult brain: a review of the clinical applications of near-infrared spectroscopy.," Philos. Transact. A Math. Phys. Eng. Sci., pp. 369:4452-4469, 2011.

[51] Arnab Ghosh,Ilias Tachtsidis, David Highton, Chris E. Cooper, Martin Smith, and Clare E. Elwella Christina Kolyva, "Cytochrome c oxidase response to changes in cerebral oxygen delivery in the adult brain shows higher brain-specificity than haemoglobin," Neuroimage., vol. 85(Pt 1), pp. 234-244., 2014.

[52] Martin M. Tisdall , Caroline Pritchard, Terence S. Leung, Arnab Ghosh, Clare E. Elwell , Martin Smith Ilias Tachtsidis, "Analysis of the Changes in the Oxidation of Brain Tissue Cytochrome c Oxidase in Traumatic Brain Injury Patients during Hypercapnoea: a Broadband NIRS Study ," Adv Exp Med Biol., pp. 701:9-14, 2011.

[53] Murad Banaji et al., Modelling of Mitochondrial Oxygen Consumption and NIRS Detection of Cytochrome Oxidase Redox State, IRUN R. COHEN NATHAN BACK, Ed.: Springer, 2010, vol. 662.

[54] Ludwig B. Richter OM, "Cytochrome c oxidase--structure, function, and physiology of a redox-driven molecular machine.," Rev Physiol Biochem Pharmacol., pp. 147:47-74, 2003.

[55] Banaji M. T, "A generic model of electron transport in mitochondria. ," heoritical Biology., 2006.

[56] B. Ludwig O.M. H. Richter, "Cytochrome c oxidase - structure, function, and physiology of a redox-driven molecular machine," Reviews of Physiology, Biochemistry and Pharmacology, vol. 147, pp. 47-74, 2003.

[57] ROGER SPRINGETT CHRIS E. COOPER, "Measurement of cytochrome oxidase and mitochondrial energetics by near-infrared spectroscopy," Phil. Trans. $R$. Soc. Lond., pp. 352, 669-676, 1997.

[58] David T. DELPY and Edwin M. NEMOTO Chris E. COOPER, "The relationship of oxygen delivery to absolute haemoglobin oxygenation and mitochondrial cytochrome oxidase redox state in the adult brain: a near-infrared 
spectroscopy study," Biochem. J., pp. 332, 627-632, 1998.

[59] Matthais Kohl, Hellmuth Obrig, RUdiger Wenzel, Wolfram von Pannwitz, *Steven 1. Matcher, Ulrich Dirnagl, tChris E. Cooper, and Arno Villringer Hauke R. Heekeren, "Noninvasive Assessment of Changes in Cytochrome-c Oxidase Oxidation in Human Subjects During Visual Stimulation," Journal of Cerebral Blood Flow and Metabolism, pp. 19:592-603, 1999.

[60] Tachtsidis I, Leung TS, Elwell CE, Smith M. Tisdall MM, "Near-infrared spectroscopic quantification of changes in the concentration of oxidized cytochrome c oxidase in the healthy human brain during hypoxemia.," J Biomed Opt. , pp. 12(2):024002, 2007.

[61] Toronov V, Elliott JT, Diop M, Lee TY, St Lawrence K. Yeganeh HZ, "Broadband continuous-wave technique to measure baseline values and changes in the tissue chromophore concentrations.," Biomed Opt Express, pp. 1;3(11):2761-70, 2012. 